

\section{DISCLAIMER}

This report was prepared as an account of work sponsored by an agency of the United States Government. Neither the United States Government nor any agency Thereof, nor any of their employees, makes any warranty, express or implied, or assumes any legal liability or responsibility for the accuracy, completeness, or usefulness of any information, apparatus, product, or process disclosed, or represents that its use would not infringe privately owned rights. Reference herein to any specific commercial product, process, or service by trade name, trademark, manufacturer, or otherwise does not necessarily constitute or imply its endorsement, recommendation, or favoring by the United States Government or any agency thereof. The views and opinions of authors expressed herein do not necessarily state or reflect those of the United States Government or any agency thereof. 


\section{DISCLAIMER}

Portions of this document may be illegible in electronic image products. Images are produced from the best available original document. 


\section{NOTICE}

This report was prepared as an account of work sponsored by the United States Government. Neither the United States nor the United States Energy Research and Development Administration, nor any of their employees, nor any of their contractors, subcontractors, or their employees, makes any warranty, express or implied, or assumes any legal liability or responsibility for the accuracy, completeness or usefulness of any information, apparatus, product or process disclosed, or represents that its use would not infringe privately owned rights.

This report has been reproduced directly from the best available copy.

Available from the National Technical Information Service, U. S. Department of Commerce, Springfield, Virginia 22161

Price: Paper Copy $\$ 5.50$ (domestic)

$\$ 8.00$ (foreign)

Microfiche $\$ 2.25$ (domestic)

$\$ 3.75$ (foreign) 


\title{
DESIGN, CONSTRUCTION, AND TESTING OF A \\ RESIDENTIAL SOLAR HEATING AND COOLING SYSTEM
}

Prepared for

Committee on the Challenges of Modern Society (CCMS)

Solar Energy Pilot Study

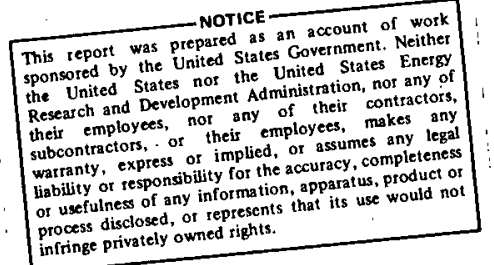

\begin{abstract}
Under the Support of the
Energy Research and Development Administration

Washington, D.C.
\end{abstract}

\author{
Prepared by - \\ George O:G. Lof \\ Dan'S. Ward
}

Solar Energy Applications Laboratory

Colorado State University

\footnotetext{
DISTRIBUTION OE THIS DOCUMENT IS UNLIATER E 
Cover Sheet. . . . . . . . . . . . . . . . . . . i

Table of Contents. . . . . . . . . . . . . . . . . . i i

List of figures... . . . . . . . . . . . . . . . . . iii

List of Tables....................... iv

Abstract ........................... . vi

General Description of System, Project, Environment. . . . . . . 1

Objectives of Project. . . . . . . . . . . . . . . 1

Description of the Environment . . . . . . . . . . . . 2

Description of System. . . . . . . . . . . . . . . 4

System Thermal Performance . . . . . . . . . . . . . . . 34

Daily, Monthly, and Annual Values of Mean Daily Heat Flows. . . 34

Quality of Thermal Performance of the System . . . . . . . 48

Solar Contribution to Energy Requirements. . . . . . . . . . 48

Monthly and Annual Energy or Fuel Savings. . . . . . . . . 53

Energy or Mass Balances. . . . . . . . . . . . . . 57

System Economic Analysis . . . . . . . . . . . 62

Total Cost of the Solar Portion of the System. . . . . . . 63

Labor Costs. . . . . . . . . . . . . . . . . . 63

Material Costs .. . . . . . . . . . . . . . 63

Operational Costs. .................... 63

Local Economic Factors . . . . . . . . . . . . . 67

Expected Selling Price or Costs. . . . . . . . . . . 67

Maintenance Frequency and Costs. . . . . . . . . . . . 67

User Reaction and Comments ................ 67

Sub-Systell Performance . . . . . . . . . . . . . 68

Solar Collectors ................ . . 68

Heat Transfer Subsystem. . . . . . . . . . . 86

Thermal Energy Storage Subsystems. . . . . . . . . 89

Air Conditioning Subsystem .............. . . 91 


\section{LIST OF FIGURES}

Figure

Page

1 Colorado State University Solar House I. . . . . . . . . . 6

2 Solar Heating and Cooling System (CSU Solar House I) . . . . . 7

3 Diagram of Piping at Collector Inlet and Outlet (in Attic) . . . 9

4 Block Diagram - Control Loop . . . . . . . . . . . . . 11

5. Location of Control Sensors. . . . . . . . . . . . 13

6 Schematic Diagram of Control Circuit . . . . . . . . . . . 14

7 Mode I - Solar Collection Using Heat Exchanger . . . . . . . 16

8 Mode 2 - Solar Collection Without Heat Exchanger . . . . . . . . 18

9 Mode 3 - Cooling Directly from Collectors. . . . . . . . . 19

10 Alternate Mode - Solar Heating with Auxiliary Boosting When Solar Cannot Carry the Heating Load . . . . . . . . 21

Data Flow Diagram.

22

12 Temperature Measurement Points on the Collector Panels . . . . 26

13 Temperature Measurement Points on Collector \#8 . . . . . . . 27

14 Temperature and Flow Measurement Points on the Mechanical System ........................ . 28

15 Sample Print Out of Daily Values of Data Collected . . . . . . 31

16 Solar Collector Cross-Section . . . . . . . . . . . 69

17 Absorber Panel Design. . . . . . . . . . . . . . 70

18 Collector Panel Tube Pattern . . . . . . . . . . . . 72

19 Collector Efficiency versus $\Delta T / H_{R}$ (Test Module). . . . . . . . 76

20 Pressure Drop Due to Friction Across One Absorber Plate as a Function of Liquid Type and Liquid Temperature. . . . .. 80

21 Measured Performance of ARKLA 3-ton Lithium Bromide Absorption Cooler. . . . . . . . . . . . . . 93

22 Evaporator Schematic . . . . . . . . . . . . . . . 95

23 Relative Contributions to Cooling Unit by Solar and Auxiliary (August 1974).. . . . . . . . . . . . . 96

24 Relative Contributions to Cooling Load by Solar and Auxiliary (nugust 1974). . . . . . . . . . . . . 99

25. Coefficient of Performance and Cooling Rate versus Time of Start Up for Absorption Cooling Unit. . . . . . . . . 101

26 Relative Contributions to Cooling Load by Solar and Auxiliary (Normal Cooling Load Only) . . . . . . . . 103 


\section{LIST OF TABLES}

$\underline{T a b l e}$

Page

Mean Monthly Values of Global (Total) Insolation on a

Plane Tilted Toward the South at a $45^{\circ}$ Angle, Dry and

Wet Bulb Ambient Temperatures, and Wind Speed. . . . . . . . 3

2 CSU Solar House I Measurement Sensors. . . . . . . . . . . . 24

3 Data Acquisition Equipment Specifications. . . . . . . . . 29

4 Daily Total Heat Rates (September 1974). . . . . . . . . 35

5 Daily Total Heat Rates (October 1974). . . . . . . . . 36

6 Daily Total Heat Rates (November 1974) . . . . . . . . . 37

7 Daily Total Heat Rates (December 1974) . . . . . . . . 38

8 Daily Total Heat Rates (January 1975). . . . . . . . . . 39

9 Daily Total Heat Rates (February 1975)........... . 40

10 Daily Total Heat Rates (March 1975)............... . 41

11 Daily Total Heat Rates (April 1975). . . . . . . . . . . 42

12 Daily Total Heat Rates (May 1975). . . . . . . . . . . 43

13 Daily Total Heat Rates (June 1975) . . . . . . . . . . 44

14 Daily Total Heat Rates (Juiy 1975) . . . . . . . . . . . 45

15 Daily Total Heat Rates (August 1975) . . . . . . . . . 46

16 Monthly and Annual Averages of Mean Daily Heat Rates. . . . . . 47

17 Daily Mean Building Temperatures . . . . . . . . . . . . 49

18 Solar Contribution to Energy Requirements of Domestic

19 Solar Contribution to Energy Requirements of Heating

(Per Cent of Load)................... 51

20 Solar Contribution to Energy Requirements of Cooling

(Per Cent of Load). . . . . . . . . . . . . . 52

21 Monthly and Annual Energy or Fuel Savings. . . . . . . . . . 54

22 Selected Energy Balances on Thermal Storage Unit . . . . . . . 58

23 Monthly and Annual Averages of Daily Mean Values

(lleating System).................... 59

24 Solar Collector Costs. . . . . . . . . . . . . . . 64

25 Solar Heating and Cooling System Costs . . . . . . . . . . . 65

26 Supplemental Energy Usage and Costs (1 September 1974

to 31. August 1975) . ............... 66

27 Mean Monthly Averages of Daily Collector Efficiencies. . . . . 78

$28 \mathrm{pH}$ and Conductivity Measurements . . . . . . . . . . . . . 85

29 Atomic Absorption Analys is of Collector Fluids . . . . . . . . 85

30 Composition of Corrosion Inhibitor . . . . . . . . . . . . 90 


$$
\text { List of Tables (con't) }
$$

Table

31 Relative Contributions to the Cooling Load by Solar and Auxiliary (August 1974) - Ten Day Totals . . . . . . . . 97 Monthly Averages of Cooling Values. . . . . . . . . . . . 105 
ABSTRACT

The NSF/CSU Solar House I solar heating and cooling system became operational on 1 July 1974. During the first months of operation the emphasis was placed on adjustment, "tuning", and fault correction in the solar collection and the solar/fuel/cooling subsystems. Following this initial check out period, analysis and testing of the system utilizing a full year of data was accomplished: This report discusses the results of this analysis of the full year of operation.

During the period 1 September 1974 through 31 August 1975, approximately 27 per cent of the total cooling load was provided by solar energy. Solar heating over the same period of time provided 76 per cent of the space heating load and 42 per cent of the domestic hot water (DHW) heating load. These percentages represent a total solar contribution of 53,140 $\mathrm{MJ} /$ year delivered to load $(14,160 \mathrm{MJ} /$ year to the cooling unit; 26,130 $\mathrm{MJ} /$ year to heating; and $12,850 \mathrm{MJ} /$ year to domestic hot water). Natural gas accounted for $63.720 \mathrm{MJ} /$ year, total.

Analysis of e data has provided several significant results associated with the operating characteristics of the solar system and the individual components. Accounting for many of these characteristics and evaluating the effects of various improvements to the solar system yields revised figures of seasonal per cent of load carried by solar. These revised values are 81 per cent of the cooling load provided by solar, 87 per cent of the space heating load, and 49 per cent of the domestic hot water load. 
GENERAL DESCRIPTION OF SYSTEM PROJECT AND ENVIRONMENT

\section{OBJECTIVES OF PROJECT}

The primary objective of the project is the design, construction, testing, and evaluation of a practical system for utilizing solar energy to drive heating, cooling; and domestic hot water subsystems, supplemented as necessary with auxiliary fuel. The project is designed to provide the following information:

1. Design data for integrated systems and the various subsystems,

2. System performance for space heating, cooling, and domestic hot water heating;

3. Operating economics,

4. Verification of the University of Wisconsin system design model,

5. Design and operation of automatic controls,

6. Correlation of standard Weather Bureau data with on-site insolation data, and

7. System reliability evaluation

System design was accomplished during the first five months (September 1973 to January 1974) and construction completed during the following five month period (February to June 1974). This report details the evaluation of the system's performance during the period 1 September 1974 through 31 August 1975. Efforts are now underway to modify and improve the performance of the solar system, and conduct a comparative evaluation of the original design and the modified system. 


\section{DESCRIPTION OF THE ENVIRONMENT}

\section{Climate}

According to Trewartha designation*, climate is classified as BSk, (dry, semi-arid [steppe], middle latitude) with Continental winter, anticyclone, and meager rainfall (mostly in summer).

Annual rain and snowfall is $36.3 \mathrm{~cm}$ (14.31 inches). This corresponds to a rainfall of $24.9 \mathrm{~cm}$ (9.82 inches) and a snowfall of $11.4 \mathrm{~cm}(4.49$ inches). The snowfall can be given in equivalent water as $114 \mathrm{~cm}(44.8$ inches). The values were computed as yearly averages over a 42-year period (1930 to 1972).

Percentage of maximum possible annual sunshine hours is 67 per cent (computed over a 57-year period).

\section{Location}

The CSU Solar House I is located at $40.6^{\circ} \mathrm{N}$ and $105.1^{\circ} \mathrm{W}$, at an altitude of 1585 meters (5200 feet) above sea level. It is positioned on the south side of a gently sloping hill, approximately 100 meters from a. large, three story building on the top of the hili (to the north-northeast). The front range of the Rocky Mountains is located about one kilometer to the west of the Solar House, and about 800 meters above the plain. The high altitude, limited rainfall, and exceptional air clarity provide a high level of solar insolation.

\section{Solar Radiation}

The mean monthly global (total) insolation on a plane tilted toward the south at a 45 degree angle is Tabulated in Table 1. The data were obtained during the period of this report (1 September 1974 to 31 August 1975) by the use of an Eppley pyranometer. The millivolt output of the

\footnotetext{
*G.T. Trewartha, An Introduction to Climate (McGraw-Hill; New York), 1954.
} 
Table 1

Mean Monthly Values of Global (Tota1) Insolation on a Plane Tilted Toward the South at a $45^{\circ}$ Angle, Dry and Wet Bulb Temperatures, and Wind Speed

\begin{tabular}{|c|c|c|c|c|c|}
\hline \multirow[b]{2}{*}{ Month } & \multicolumn{2}{|c|}{$\begin{array}{l}\text { Solar Insolation } \\
\text { on a } 45^{\circ} \text { Surface }\end{array}$} & \multicolumn{2}{|c|}{ Ambient Temperature } & \multirow{2}{*}{$\begin{array}{l}\text { Wind } \\
\text { Speed } \\
(\mathrm{m} / \mathrm{sec})\end{array}$} \\
\hline & $\left(\frac{\text { Langleys }}{\text { day }}\right)$ & $\left(\frac{M J}{m^{2} \cdot d a y}\right)$ & $\begin{array}{c}\text { Dry Bulb } \\
\left({ }^{\circ} \mathrm{C}\right)\end{array}$ & $\begin{array}{l}\text { Wet BuTb } \\
\left({ }^{\circ} \mathrm{C}\right)\end{array}$ & \\
\hline September & 370 & 15.5 & 16.1 & * & * \\
\hline October & 284 & 11.9 & 11.2 & * & * \\
\hline November & 261 & 10.9 & 2.2 & * & * \\
\hline December & 320 & 13.4 & -1.9 & * & * \\
\hline January & 334 & 14.0 & -1.4 & * & 3.2 \\
\hline February & 426 & 17.8 & -1.7 & * & 2.0 \\
\hline March & 372 & 15.6 & 2.4 & * & 3.8 \\
\hline April & 506 & 21.2 & 6.3 & * & 4.1 \\
\hline May & 407 & 17.0 & 12.5 & * & 4.8 \\
\hline June & 460 & 19.3 & 17.8 & 15.6 & 5.1 \\
\hline Juty & 493 & 20.6 & 23.7 & 18.2 & 4.3 \\
\hline August & 478 & 20.0 & 22.0 & 17.3 & 4.6 \\
\hline
\end{tabular}

${ }^{\star}$ Not recorded 
pyranometer was integrated over two-minute periods and the two-minute totals recorded on magnetic tape.

Ambient Temperatures

Mean monthly dry and wet bulb temperatures are tabulated in Table 1. Based on these data, the mean annual heating degree days are $3303^{\circ} \mathrm{C}$ days $\left(18^{\circ} \mathrm{C}, 65^{\circ} \mathrm{F}\right.$ base), while the mean annual cooling degree days are $422^{\circ} \mathrm{C}$ days $\left(18^{\circ} \mathrm{C}, 65^{\circ} \mathrm{F}\right.$ base). Design temperatures (for the Denver area, $100 \mathrm{~km}$ to the south) are $-23^{\circ} \mathrm{C}\left(-10^{\circ} \mathrm{F}\right)$ for winter and $35^{\circ} \mathrm{C}\left(95^{\circ} \mathrm{F}\right)$ dry bulb, $18^{\circ} \mathrm{C}$ $\left(64^{\circ} \mathrm{F}\right)$ wet bulb for the summer.

Measurement equipment includes a copper constantan thermocouple (shaded from the sun) for dry bulb temperatures, and a dew point hydrometer. for obtaining dew point temperatures for computation of wet bulb temperatures. The hygrometer has an intake pipe for sensing ambient (outdoor) dew point. Accuracy has been determined to be within $0.5^{\circ} \mathrm{C}$.

Wind

Average monthly wind speed is tabulated in Table 1. Data were obtained from a wind speed cup aeronometer, mounted on the CSU Solar House I roof ridge. The data are intergrated over five-minute periods to obtain total wind run.

\section{DESCRIPTION OF SYSTEM}

Qualitative Description

The solar heating and cooling system was installed in a residencetype building at a site on the Foothills Campus of Colorado State University in Fort Collins, Colorado. The building is a modern three-bedroom frame residence with a living area of 140 square meters ( 1500 square feet) and 
a full, heated basement, the south wall of which is entirely above grade. The design heating load was computed to be 16.1 kilowatts at $-23^{\circ} \mathrm{C}(55,000$ $\mathrm{Btu} / \mathrm{hr}$ at $-10^{\circ} \mathrm{F}$; corresponding to $17,600 \mathrm{Btu} /{ }^{\circ} \mathrm{F}$ day). The design cooling load is approximately 10.5 kilowatts ( 3 tons or $36,000 \mathrm{Btu} / \mathrm{hr}$ ). The insulation was typical, with $8.9 \mathrm{~cm}$ (3.5 inches) fiberglass in the walls $\left(R=6.85 \frac{{ }^{\circ} \mathrm{Cm}^{2}}{\mathrm{~W}} ; 12 \frac{\mathrm{Hr}-{ }^{\circ} \mathrm{F}-\mathrm{ft}^{2}}{\mathrm{Btu}}\right)$ and $14 \mathrm{~cm}$ ( 5.5 inches) of fiberglass in the ceiling $\left(R=10.85 \frac{{ }^{\circ} \mathrm{Cm} 2}{\mathrm{~W}} ; 19 \frac{\mathrm{Hr}-{ }^{\circ} \mathrm{F}-\mathrm{ft}^{2}}{\mathrm{Btu}}\right)$ (see Figure 1$)$.

The solar heating and cooling system includes the conventional components such as the lithium bromide absorption cooling unit, hot water boiler, air heater coil, hot water heater, and associated piping, ducts and pumps; and the solar components, consisting of a solar collector and pump, thermal storage with heat exchanger and hot water preheat tank, and an automatic valve. Figure 2 is a cross-section schematic diagram of the installation which shows all of the components except the collector and associated piping, the control sensors, and the air distribution system. The primary modes of solar heat collection are: storing heat from the solar collector via a heat exchanger, and storing heat directly from the solar collector. A third mode of solar collection is the supply of heated fluid directly from the solar collector to the heating unit. Energy to the heating or cooling unit is provided either by use of hot water from storage, if the temperature is adequate, or from the auxiliary boiler as necessary.

An alternate heating mode utilizes whatever heat is in storage, even at temperature as $10 \mathrm{w}$ as $27^{\circ} \mathrm{C}\left(80^{\circ} \mathrm{F}\right)$, with the auxiliary boiler supplying hot water to an auxiliary air heating coil. In this mode the auxiliary boiler acts as a temperature booster for the solar system. In all other modes of operation, either the auxiliary or solar is used, never the two together. 


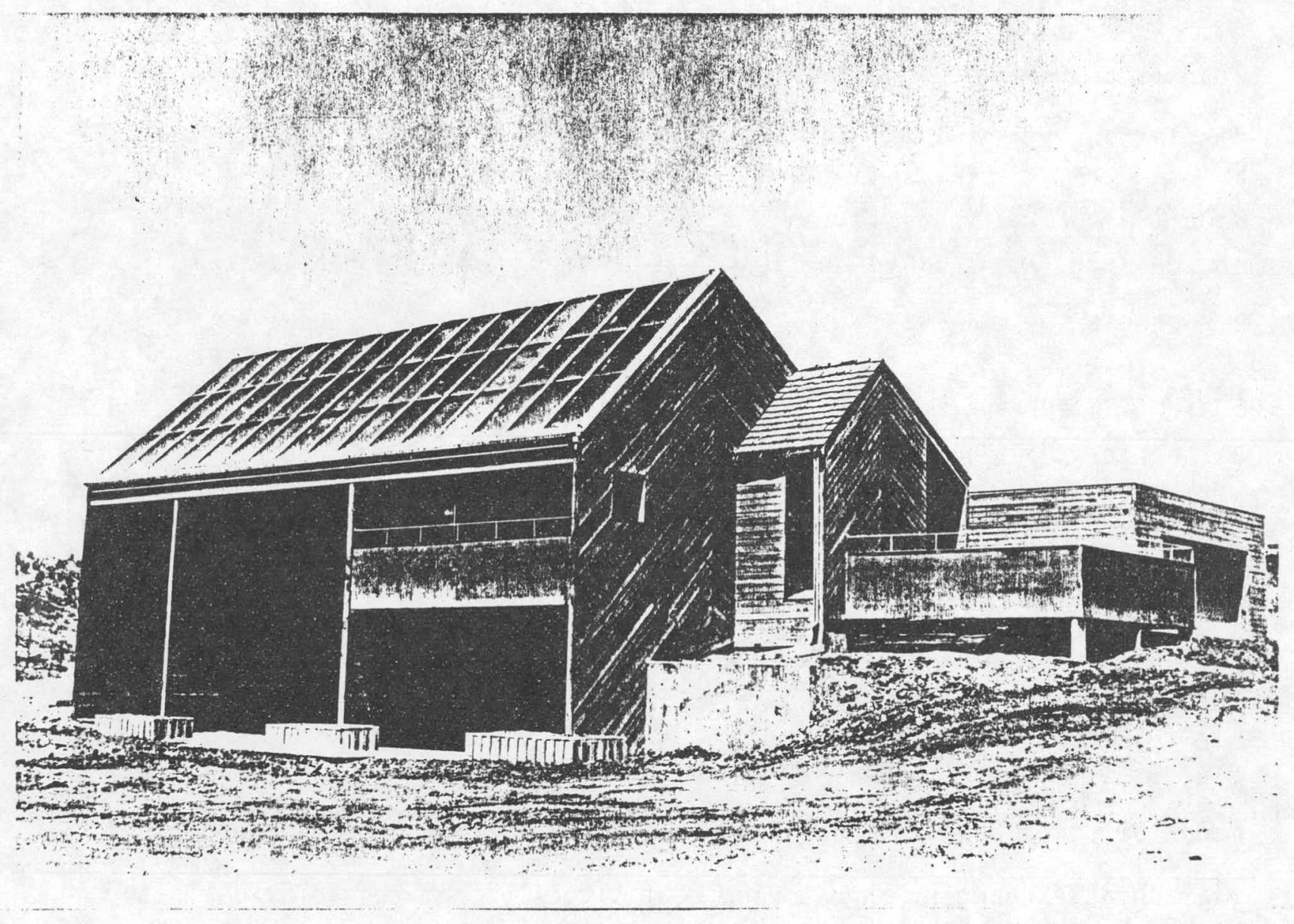

Figure 1. Colorado State University Solar House I 
SOLAR ENERGY HOUSE

Heating - Air Conditioning - Hol Woter

Equipment

To Colleciors

From Collectors

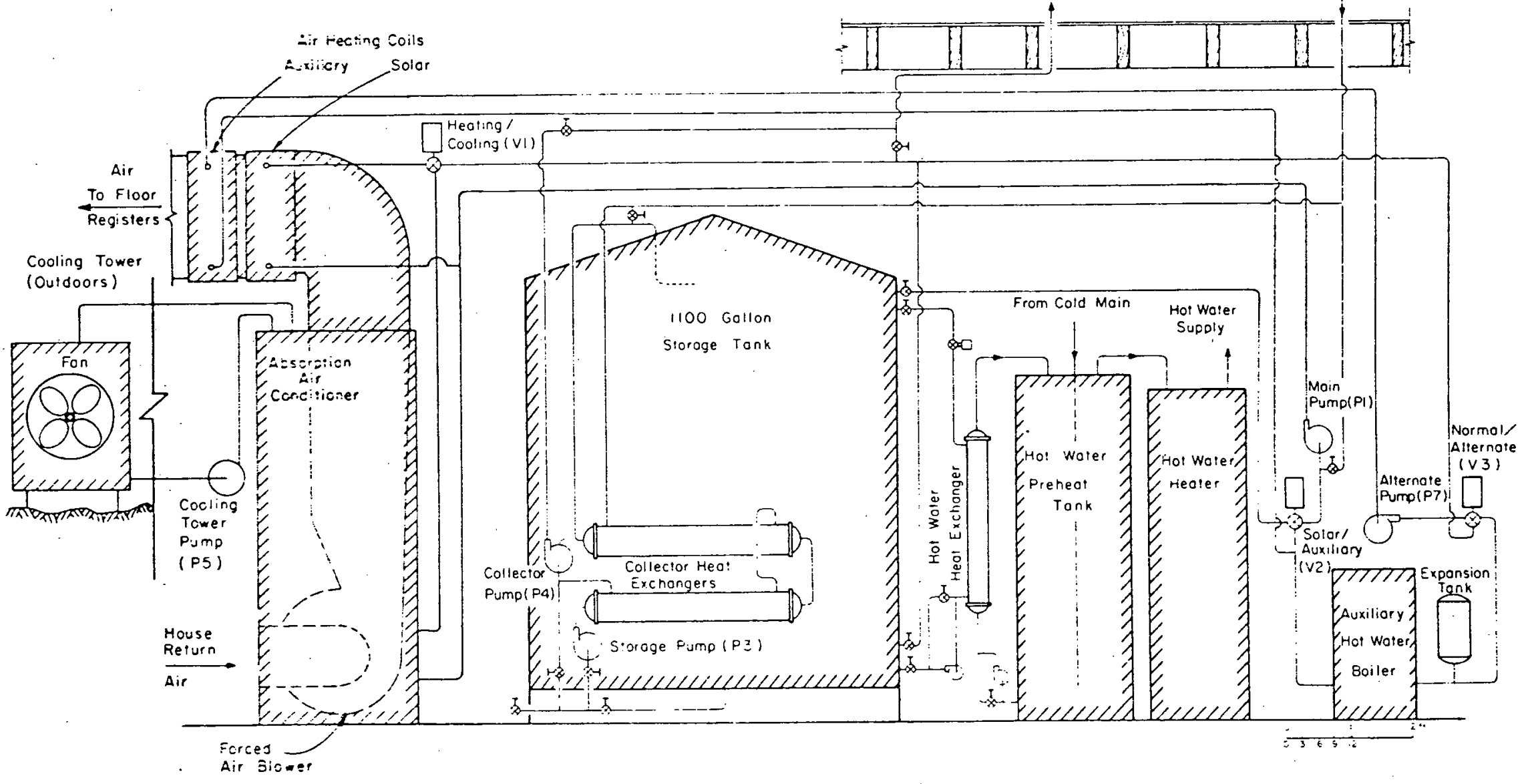

\section{Airicry sols}

Figure 2

Solar Heating and Cooling System 
The house hot water system utilizes solar storage for preheating service hot water using a tube and shell heat exchanger. Water from a cold water main enters the preheat tank, to which heat is supplied from solar storage by pumped circulation through the heat exchanger. On demand, the preheated water then flows to a conventional gas hot water heater, which maintains the required temperatures.

Because of the possibility of freeze damage by circulating water through the collector, normal operation provides solar heat collection in a 60 per cent solution of ethylene glycol (commercial automotive antifreeze) in water. The cost of several hundred gallons. of glycol in the main storage system would be prohibitive, so a heat exchanger (a series of two tube-andshell units) is employed for transfer of heat from the small volume of collector fluid (about 106 liters, 28 gallons) to a large volume of water comprising the thermal storage.

Figure 3 details the interface between the collector panels and the rest of the solar system. The collector absorber panels are made of aluminum, and to avoid corrosion they must therefore not be directly connected to the copper piping in the solar system. Rubber hose connections between the copper and aluminum piping accomplish. this separation. A filter and aluminum screen "getter" (sacrificial) are also used to minimize corrosion risk.

A 113 liter (30 gallon) vented surge tank is installed on the outlet side of the collector. This tank provides volume for liquid expansion and also permits boiling to occur in the collector without pressure buildup. If the collector pump fails, boiling results in some liquid loss, but the surge tank can refill the system automatically when operation resumes.

The storage container is a light gauge vertical galvanized steel cylinder 1.83 meters ( 6 feet) high and 1.68 meters ( 5.5 feet) in diameter. 
DIAGRAM OF PIPING

AT COLLETOR INLET AND OUTLET

(IN ATTIC)

I Monual Volue

Normal Flow Path

Alternate Flow Poth

This Portion Located of

Level of Attic Floor
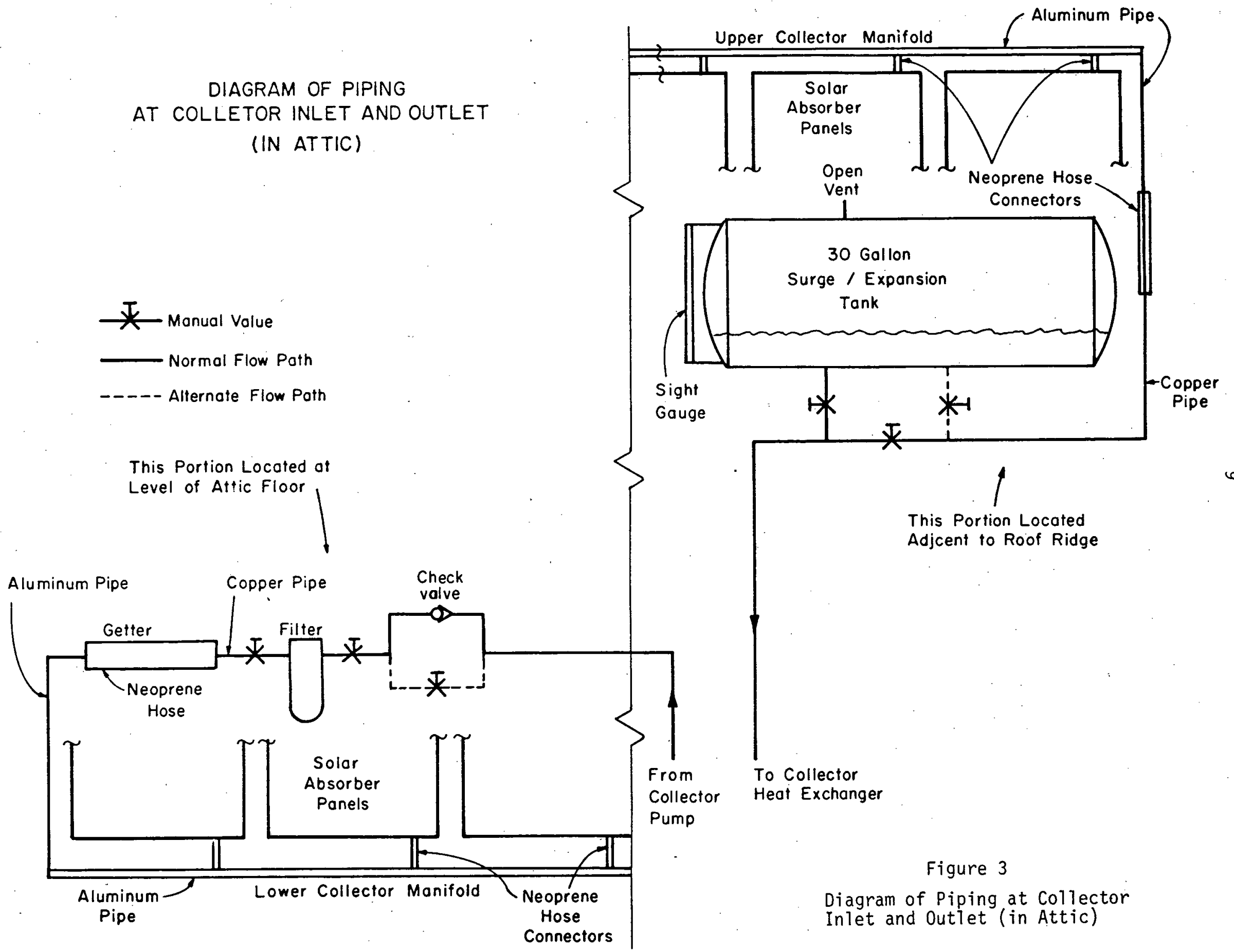

Figure 3

Diagram of Piping at Collector Inlet and Outlet (in Attic) 
It holds 4,275 liters (1131 gallons), a nominal 61 liters $/ \mathrm{m}^{2}$ of collector ( $\left.1.5 \mathrm{gallons} / \mathrm{ft}^{2}\right)$. During the first year of operation and during the data collection period covered by this report, the tank was covered by two layers of bonded glass fiber double-faced batt insulation having an $\mathrm{R}$ factor of $17.7 \frac{{ }^{\circ} \mathrm{Cm}^{2}}{\mathrm{w}}\left(30.4 \frac{\mathrm{Hr}-\mathrm{ft}^{2}-{ }^{\circ} \mathrm{F}}{\mathrm{Btu}}\right)$.

\section{QUANTITATIVE DESCRIPTION}

The heating and cooling system was designed to furnish 75 per cent of the space heating and 75 per cent of the cooling load demands of the solar house. Calculations by the University of Wisconsin showed that this performance should be obtainable with a gross collector area of 71.4 square meters (768 square feet) facing due south (i.e., the angle, $\theta$, between the projection of the sun's rays, at solar noon, on the horizontal plane and the projection of the normal to the collector on the horizontal plane is zero) at an inclination of 45 degrees (i.e., the angle between horizontal and normal to the collector), when combined with a heat storage of 4,275 liters (1131 U.S. gallons) of water.

\section{Control System Description}

CSU Solar House I utilizes a fully automatic control system for the operation of the solar heating and cooling system. Extraction of heat from the solar collectors and distribution of heat to the heating, cooling, and hot water loads are automatically controlled in response to preset temperatures or temperature differentials. This is accomplished by feedback control loops consisting of sensors, thermostats, controlled elements and the mechanical system itself. Figure 4 illustrates a typical feedback control loop. The sensors are vapor expansion bulb and capillary aquastats for the liquid temperatures, electrical resistance (thermistor) 


\section{BLOCK DIAGRAM - CONTROL LOOP}

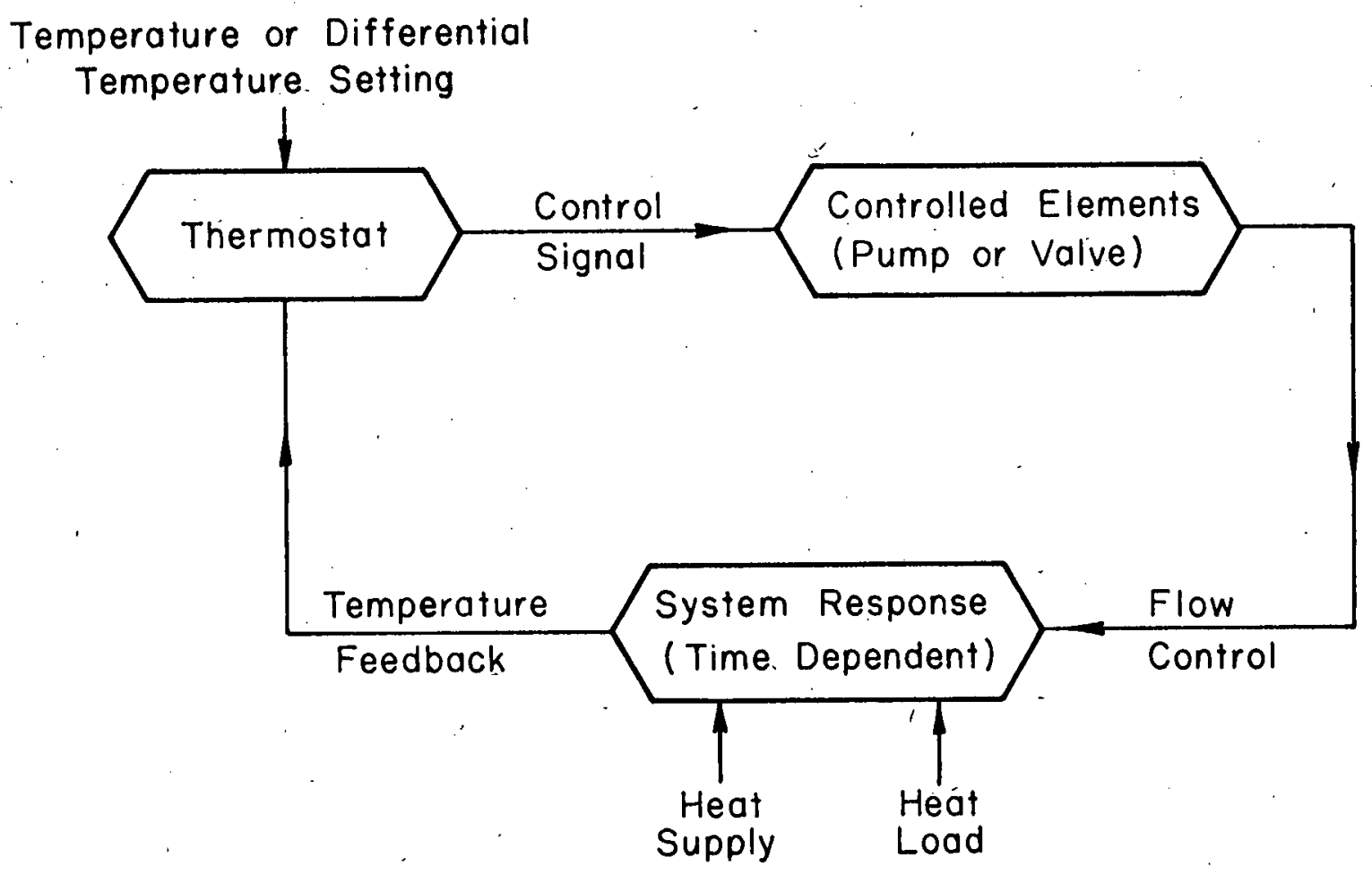

Figure 4

Block Diagram - Control Loop 
elements for collector absorber plate temperature, and a bimetal coil for air temperature in the house. Thermostats are used to compare the sensor outputs with the corresponding temperature or differential temperature settings. When the sensor output is sufficiently different from the setting (or is outside of the deadband), a control signal at 24 volts is transmitted from the thermostat to a power relay. The relay opens or closes the 110 volt power line to a controlled element which is a pump, motor, or an automatic electric valve positioner, or an air fan motor. Figure 5 shows the location and identity of the control sensors in the system.

The mechanical system is the final link in the feedback control loop. It is affected by controlled elements which regulate flow to the various components. Operation of the solar air heating coil, for example, depends on the conditions of the main system pump (PI) and the heating/cooling. automatic valve (VI). The mechanical system produces an output measured as temperature by the control sensor, thus completing the feedback control loop. Through operation of the air coil, for example, house air temperature change is sensed by the wall thermostat. All control action is of the on/off type with adjustable set points and deadbands.

Figure 6 is a schematic diagram of the control circuit. The lower portion of the diagram is the 24 volt wall thermostat primary control. circuit. Relays are actuated from the 24 volt circuit which switch power to the controlled elements illustrated in the upper portion of the drawing. The relays are designed to provide the logic required for each control function. Relays in series comprise "AND" logic, while relays in parallel provide "OR" logic. An example of "AND" control is the hot water preheat signal to pumps $P 2$ and P6 from sensors \#1 and \#2 (top line in diagram). Power is applied to these pumps to circulate storage water and service water 


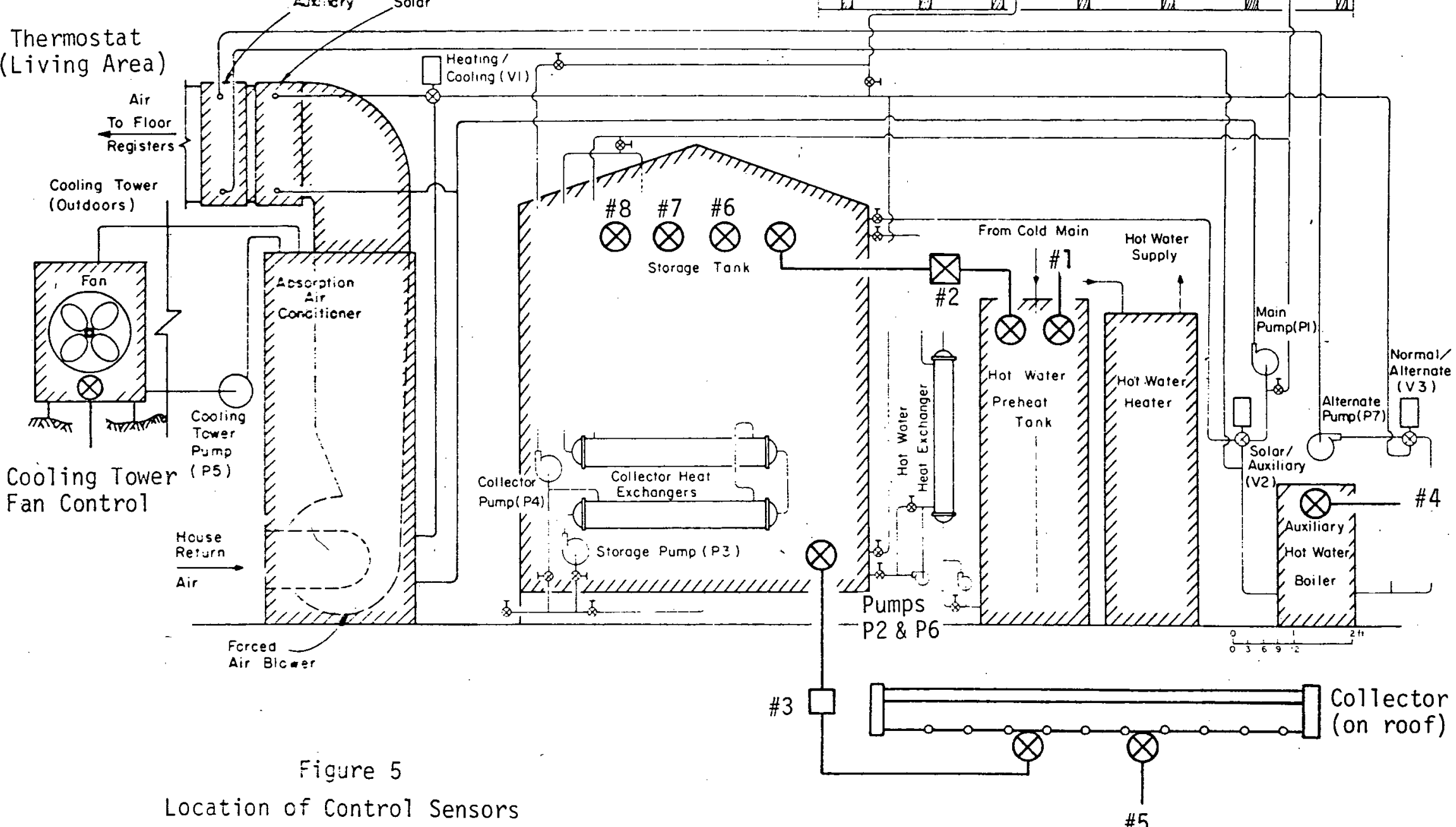




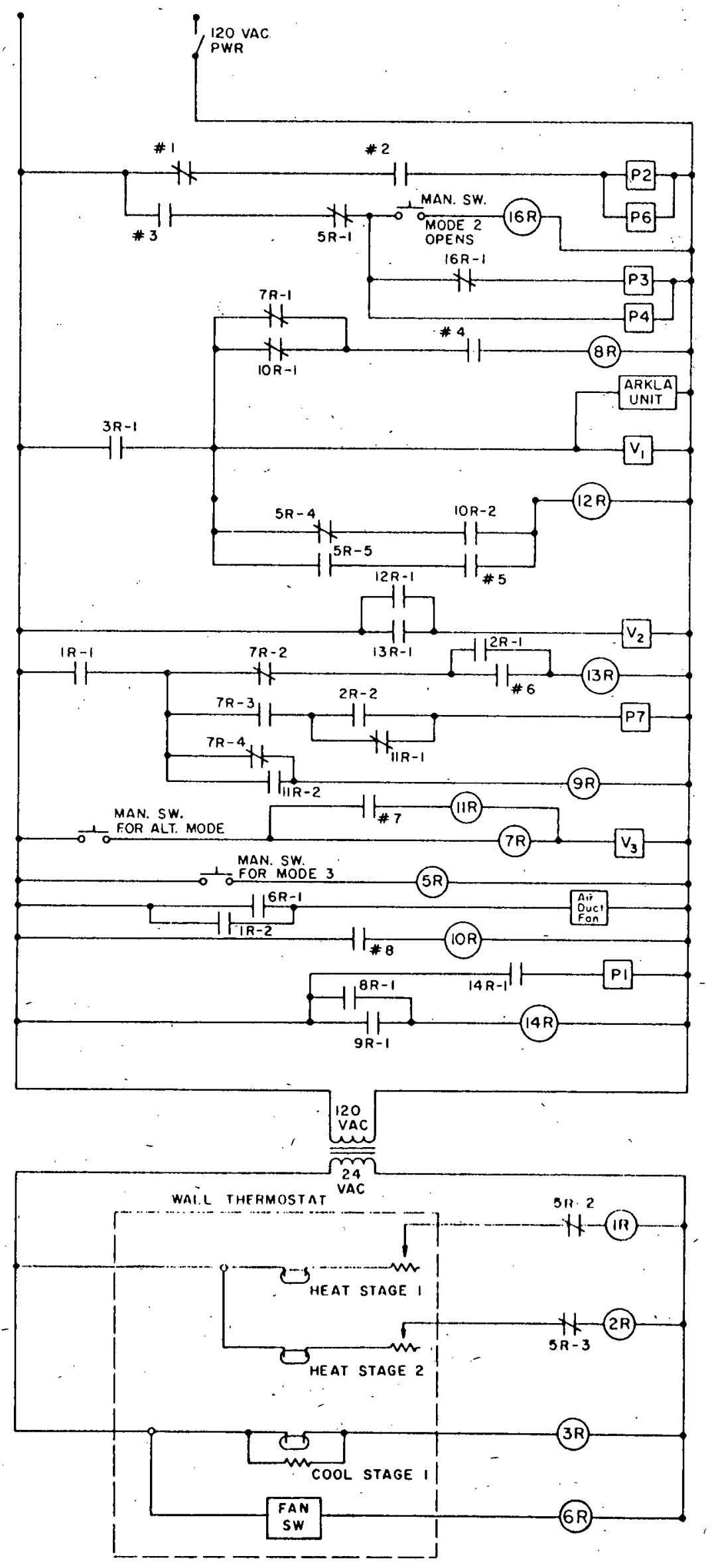


through the heat exchanger if the preheat tank temperature is below a set point (sensor \#1) AND if the temperature of storage is a preset amount above the preheat tank temperature (sensor \#2). This control is accomplished by two relays in series with control signals from the corresponding temperature sensors. An example of "OR" logic is the control of the air duct fan. (near the bottom of upper section of diagram). The fan is powered for heating $O R$ cooling, and is turned on by either of two relays in parallel; one closes upon heating demand $(1 R-2)$ and the other for cooling (3R-2).

The control system in CSU Solar House I is more complex than would be required for a single mode system. The multi-mode design was chosen to provide versatility of operation. A mode is selected manually and the control system automatically assumes control in that mode. The available modes include:

(a) Mode 1 - Mode 1 is characterized by the use of a heat exchanger which separates the collector fluid from the storage tank filuid. Figure 7 illustrates with bold Iines, the fluid circuits used in Mode 1. The collector fluid is pumped (pump P4) through the shell side of a commercialiy available shell-and-tube heat exchanger, while storage fluid is pumped through the tubes by another pump (pump P3). Two main advantages of Mode 1 are the avoidance of antifreeze in the storage system and the use of non-pressurized (vented to the atmosphere) storage tank. Provision of the heat exchanger in Mode 1 permits use of only 57 liters (15 gallons) of antifreeze in the collector loop. The storage tank then needs only water and a corrosion inhibiting additive. The second advantage of Mode $i$ is a pressurized collector circuit and a non-pressurized storage tank, with the heat exchanger acting as the pressure barrier. There are two reasons for preferring a pressurized collector loop. Under pressure, the boiling temperature of the collector fluid is elevated, allowing for higher operating temperatures 


\section{- Collector Fluid
$\ldots \ldots$ - Storage Tank Fluid}

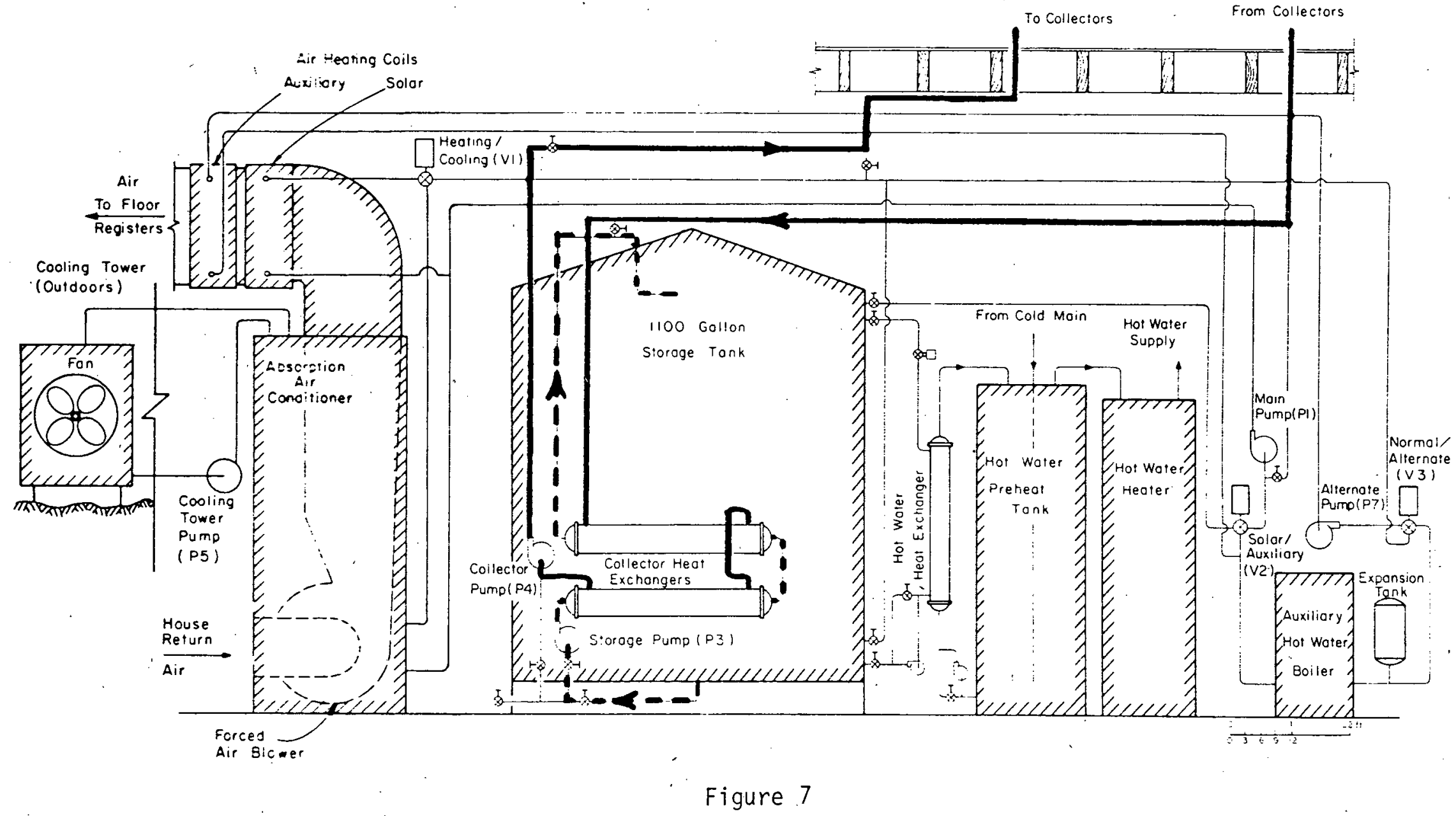

Mode 1 - Solar Collection Using Heat Exchanger 
in the collector. With the heat exchanger preventing pressure in the storage tank, the collector loop can be completely fluid filled. Consequently, there is no gravity head loss for the pump to overcome, only frictional head loss.

A third advantage of Mode 1 concerns corrosion protection of the collector absorber plates. The need for continuous filtration and deionization of the collector fluid is a major consideration. Mode 1 requires only the 106 liters (28 gallons) filling the collector loop to receive such treatment rather than the additional 4275 liters (1130 gallons) of fluid in storage.

(b) Mode 2 - Mode 2 provides collection of solar heat in water which is run directly to the storage tank. It does not utilize the collector heat exchangers nor the storage pump required in Mode 1. Figure 8 illustrates the fluid circuit for collection of solar heat in Mode 2.

Two advantages are recognized with Mode 2. There is the saving of capital and maintenance costs for the collector heat exchanger and the storage pump. A second advantage of Mode 2 is the elimination of the temperature drop across the collector heat exchangers (ranging from $0^{\circ}$ to $6^{\circ} \mathrm{C}$ ). The net result is an improvement in system efficiency.

(c) Mode 3 - Mode 3 is a cooling design which does not utilize heat storage but supplies solar heated fluid directly to the generator of the air conditioner. Figure 9 is a diagram of this mode.

The advantages of Mode 3 are higher water temperatures available to the generator of the air conditioner and the avoidance of some heat loss into the house from the storage tank. Heat loss from the storage tank in summer is particularly detrimental because it has a duel effect on cooling operation. Not only is less heat available to the generator, but heat lost into the house is an added cooling load which must be met by the air conditioner to maintain the desired house temperature. 


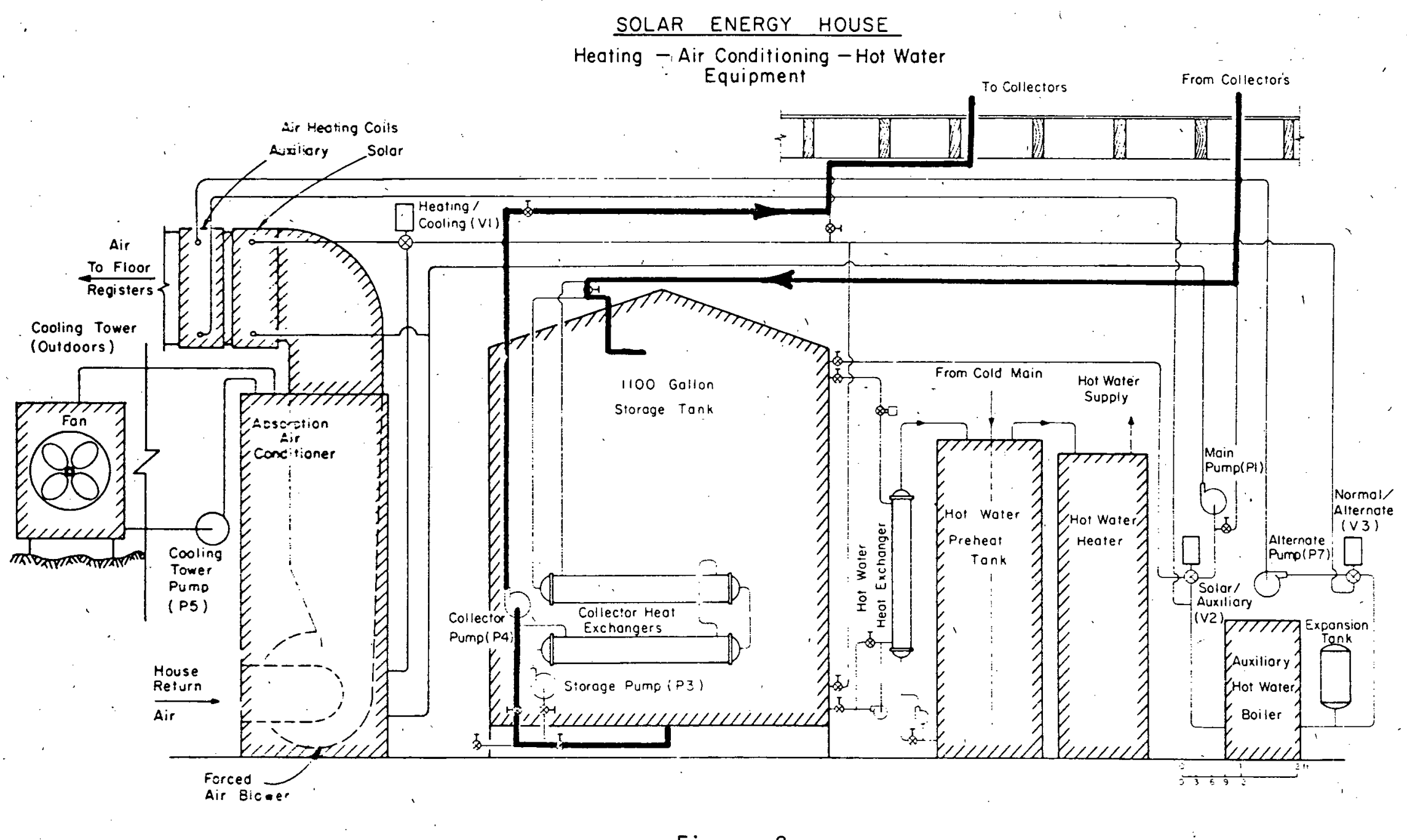

Mode. 2 - Solar Collection Without Heat Exchanger 


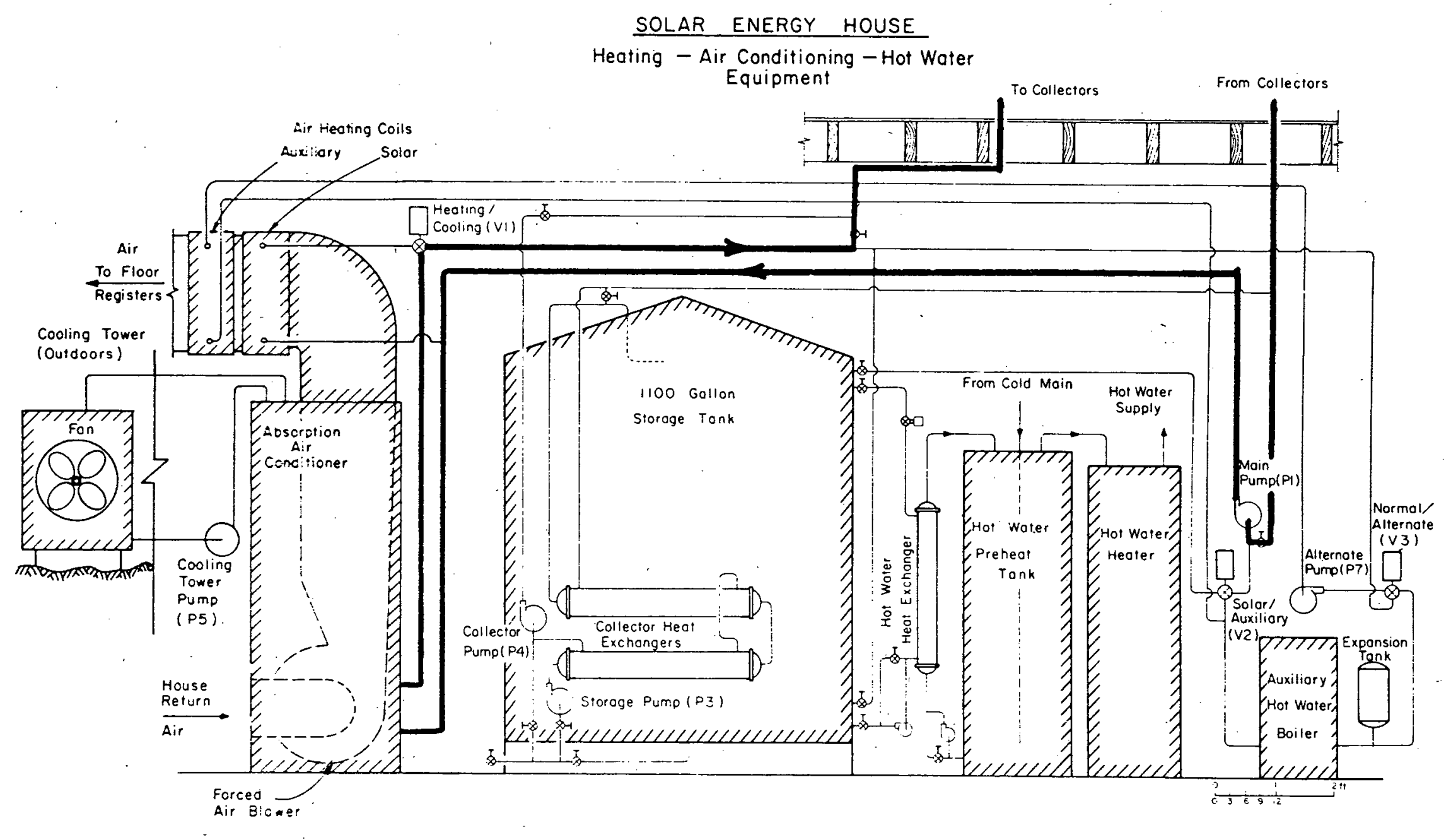

Mode 3 - Air Conditioning Directly from Collectors 
Alternate Mode - In the heating season, there will often be a condition in which the storage tank temperature is considerably above house temperature but is not high enough to carry the entire heating load (maintain the desired house temperature setting). It is particularly desirable to use this heat at moderately low temperature because it is acquired at high collector efficiency. The liquid-to-air heating design allows for separate solar storage and auxiliary boiler loops to supply heat in the air duct. This arrangement is illustrated in Figure 10. The solar air heating coil is placed ahead of the auxiliary air heating coil in the direction of air flow. The solar coil thus preheats the air while the auxiliary coil boosts the air temperature to that required to maintain the heating demand. The net result is the use of more solar and less auxiliary heating.

\section{Data Acquisition and Handling System}

The Colorado State University Solar House has been equipped with a data acquisition system to gather 74 channels of data on climatic conditions, heating loads, and equipment performance. The system is expandable to 100 channels and records up to two channels per second. Millivolt (and microvolt) analog levels from all of the measurement sensors are digitized ( $A$ to D conversion) and presented in three output forms. A digital display on the front of the instrument allows instantaneous investigation of a single selected measurement. A paper tape print-out provides observation of all the data whenever desired. Finally, the data are continuously recorded on magnetic tape. The magnetic lape serves as storage for the data unti] transferred into the computer for processing. Figure 11 illustrates the flow of measurement data through the data acquisition system.

All the measurements are made at five-minute intervals except where conditions require faster speed (up to two channels per second). Parameters which do not undergo rapid changes such as temperature and circulation flow 
- Solar

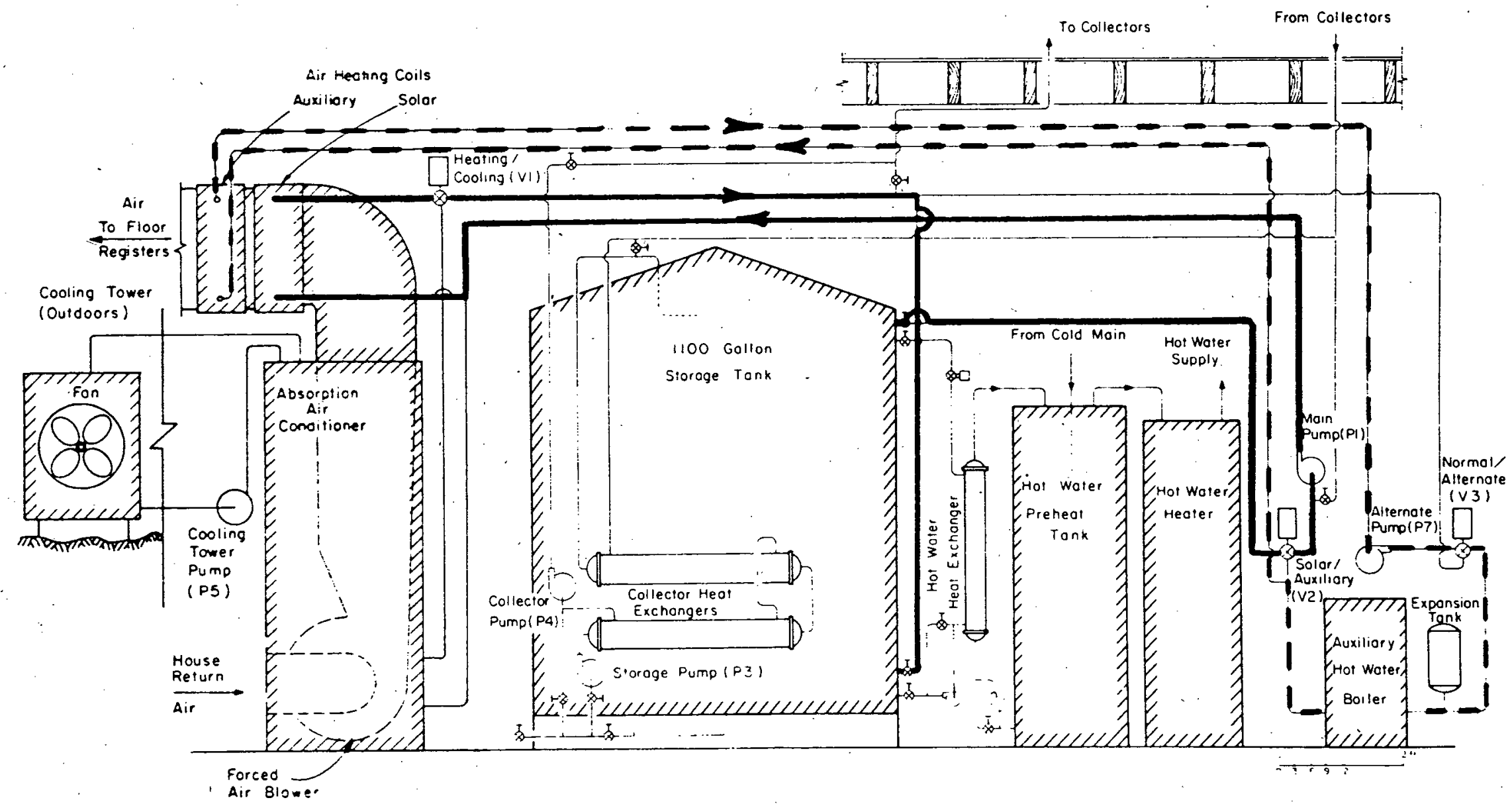

Figure 10

Alternate Mode - Solar Heating with Auxiliary Boosting When Solar Cannot Carry the Heating Load 


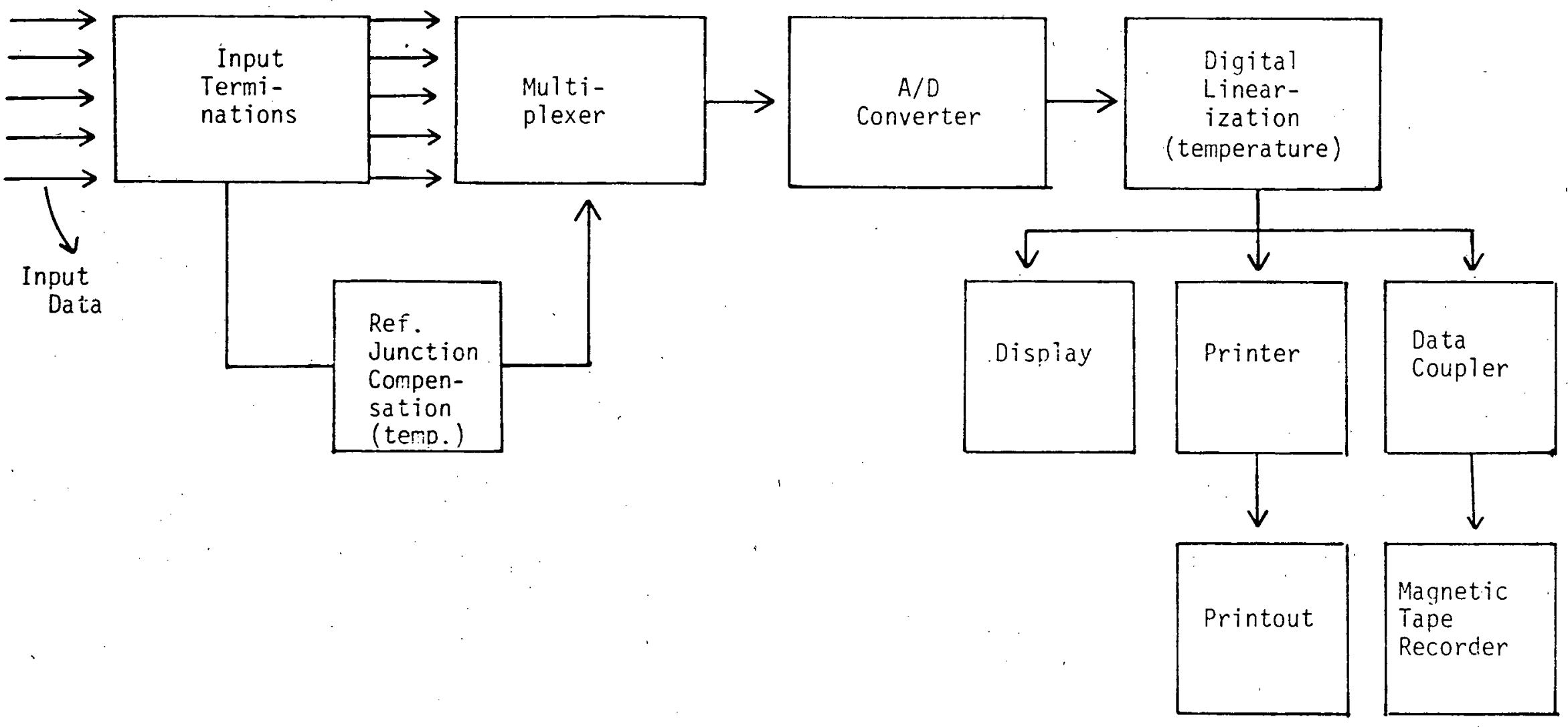

Figure 11

Data Flo:: Diagram. 
rates are observed instantaneously each five minutes and used to compute performance (such as heat transfer rate) at that instant. Parameters which. may vary widely and rapidly, such as solar radiation and hot water demand, are integrated electronically and observed as totals at the end of each five-minute period.

A listing of the measurement sensors is provided in Table 2. Temperatures are measured by copper-constantan thermocouples. The data logger. has built-in zero reference and ROM linearization. Thus the values of temperature are read and recorded directly in ${ }^{\circ} \mathrm{C}$. The millivolt function includes all measurements other than temperatures. These values must be scaled by the data handling program in the computer to arrive at engineering units. ON/OFF function indicators are used for identification of vaive positions and pump operations.

Solar radiation is measured simultaneously with two Eppley pyranometers which have been calibrated by the Eppley factory and checked by the National Bureau of Standards immediately before installation. The pyranometers are mounted at the ridge of the roof above the collectors, one in a horizontal position and the other facing south at $45^{\circ}$ (the collector angle). The redundancy in solar radiation measurement permits more direct and accurate collector input determination as well as the customary horizontal record. A back-up to this critical measurement is also thus provided. Table 3 lists the Data Acquisition Equipment Specifications.

The information which describes total system performance is processed on a one or two-day interval by transfer from the magnetic tape files into the Colorado State University CDC 6400 for computation. The tape files are stored for future reference and for more detailed analyses. Data handling is accomplished by a computer program which converts these records to desired values and compiles them from the data. Hourly averages and 
Table 2

-SOLAR HOUSE MEASUREMENTS

\section{LOCATION}

(see Figures 12, 13, 14)

A. Copper-Constantan Thermocouples

Collector Inlet

Collector Outlet

collector \#1 Outlet

Coll-ctor \#2 Outlet

Collector \#3 Outlet

Collector \#4 Outlet

Collector \#5 Outlet

Collector \#6 Outlet

Collector \#7 Outlet

Collector \#8 Outlet.

Collector \#9 outlet

Collector \#10 outlet

Collector \#11 Outlet

Collector \#12 Outlet

Collector \#13 outlet

Collector \#14 Outlet

Collector \#15 Outlet

Collector \#16 Outlet

Collector \#8 Inlet

Collector \#8

Collector \#8

Collector \#8

Collector \#8

Collector \#8

Collector \#8

Collector \#8

Collector \#8

Collector \#8

Collector \#B

Collector 18

Collector $\# 3$

Collector \#8

Storage Tank Top

Storage Tank Middle

Storage Tank Bottom

To Collector

From Collector

To Load

From Load

To Alternate Coi]

From Alternate Coil

Supply Air
TC I

TC 0

TC 1

TC 2

TC 3

TC 4

TC 5

TC 6

TC 7

TC 8

TC 9

TC 10

TC 11

TC 12

TC 13

TC 14

TC 15

TC 16

TC 17

TC 18

TC 19

TC 20

TC 21

TC 22

TC 23

TC 24

TC 25

TC 26

TC 27

TC 28

TC 29

TC 30

SST

SSM

SSB

TTC

TFC

TTL

TFL

TTAL

TFAC

TSA 
Table $2\left(\operatorname{con}^{\prime} t\right)$

LOCATION

Return Air

To Cooling Tower

From Cooling Tower

Preheat Tank

Boiler Flue

Hot Water Flue

To Service Hot Water

From Service Hot Water

House Dry Bulb

Outdoor Dry Bulb

Wet Bulb
NAME

TRA

TTCT

TFCT

$T P$

THW

THWF

TTSHW

TFSHW

THD

TOD

TWB
DATA CHANHEL

42

43

44

45

46

47

48

50

57

01

56

B. Millivolt Measurements

Collector. Flow

Alternate Coil Flow

FC

FAC

70

Cooling Tower Flow

Load Flow

Hot Water Flow

FCT

FL

FHW

71

72

73

74

$\mathrm{SH}$

60

Solar Horizontal

$S 45^{\circ}$

61

HWGAL

HWGAS

BGAS

SELEC

HELEC

WIND

62

63

64

65

66

Wind Run

67

D. On/Off Function Indicators

Heat/Cool (VI) Position

Solar/Auxiliary (V2) Position

VI

V2

Normal/Alternate (V3) Position

Hygroneter Valve Position

V3

HV 


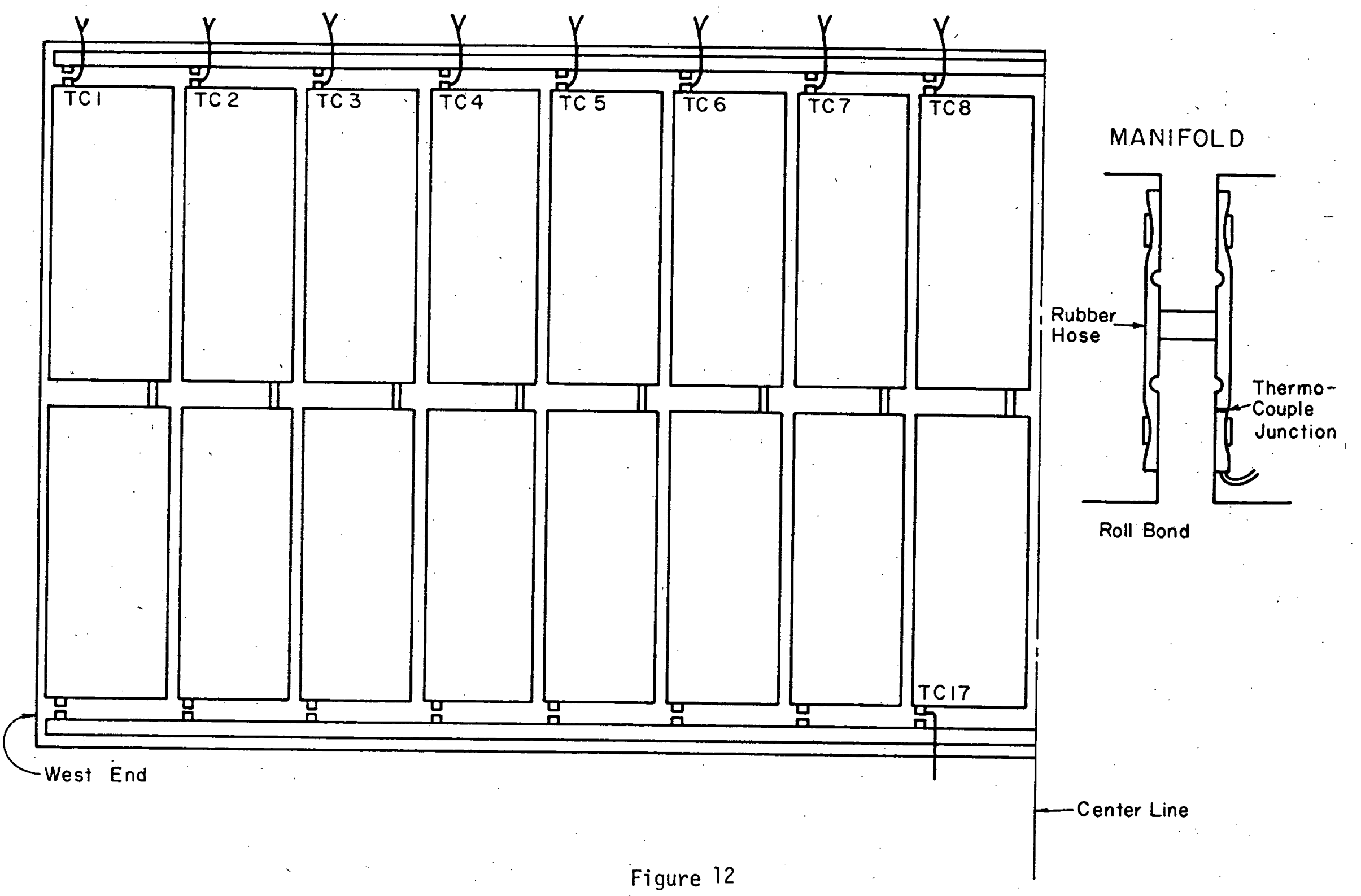

Temperature Measurement Points on the Collector Panels 

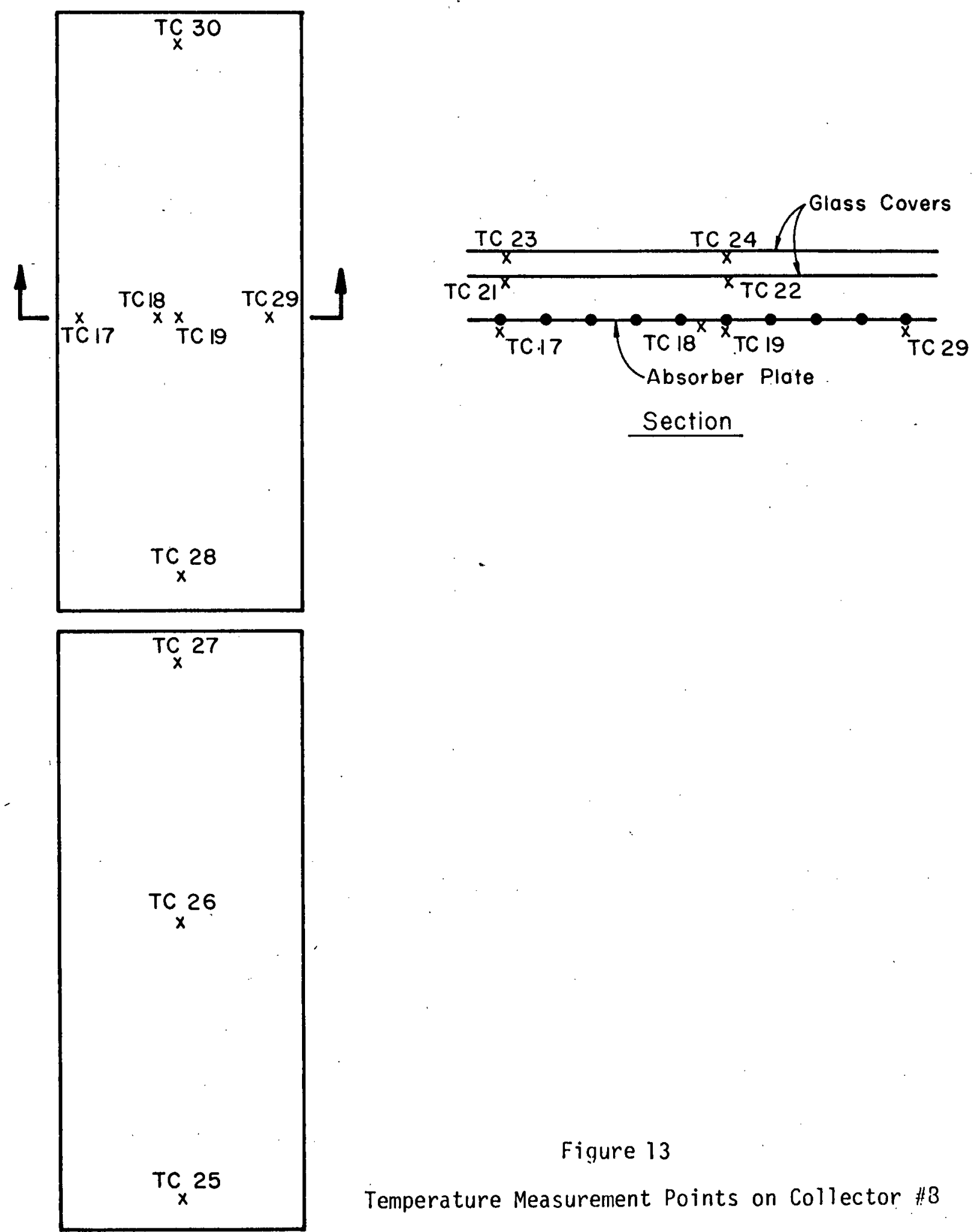

Figure 13

Temperature Measurement Points on Collector $\# 3$ 


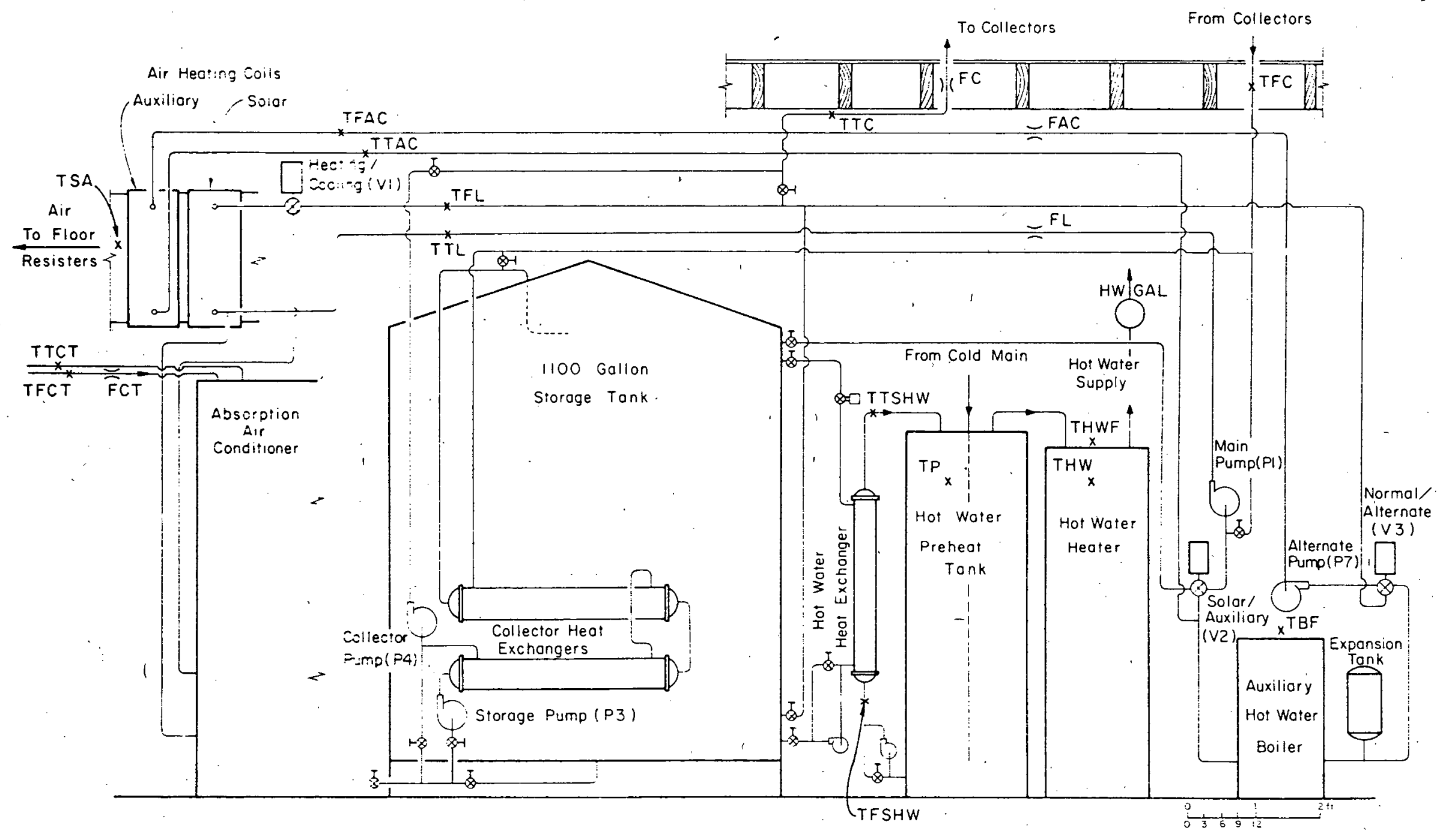

Figure 14

Temperature and Flow Measurement Points on the Mechanical System 
Table 3

Data Acquisition Equipment Specifications

Data Logger

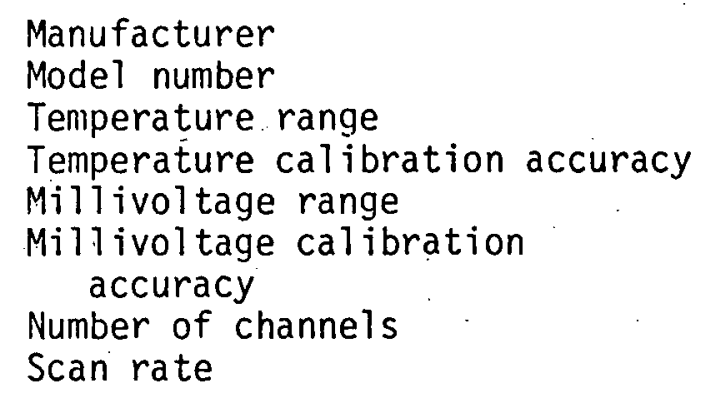

Magnetic Tape Recorder

Manufacturer

Model

Tape

Data density

Pyranometers

Manufacturer

Mode]

Accuracy

Thermocouples

Manufacturer

Model

Type

Flowmeters

Sensor manufacturer

Orifice plates and sensor cases made

by CSU machine shop, calibration accuracy

\section{Integrators}

Solar, electricity, and natural gas integrators made by CSU electronics shop, calibration accuracy
Doric Scientific

210

-190 to $+400^{\circ} \mathrm{C}$

$\pm .3^{\circ} \mathrm{C}$

$\pm 200 \mathrm{Mv}$

$\pm .004 \mathrm{Mv}$

100

2 channels/second

Kennedy

1600

7 track, $1 / 2$ inch 556 bits/inch

Eppley Laboratories, Inc. 8-48

$\pm 1 \%, 0-2.0 \mathrm{cal} / \mathrm{cm}^{2}-\mathrm{min}$

Thermo-Electric

41403

Copper-Constantan

Honeywel1

$\pm 3 \%$

$\pm 2 \%$ 
totals are printed for selected days, and daily and monthly averages and totals for all days are printed. Figure 15 is a sample print out of the daily values thus obtained. 


\begin{tabular}{|c|c|c|c|c|c|c|c|c|c|c|c|c|c|c|c|c|}
\hline \multicolumn{3}{|c|}{ DATE. } & 2sing & nEl:Ge & $\begin{array}{c}\text { fot } \\
\text { GSHE }\end{array}$ & $\begin{array}{l}R \Delta ; E S \quad \\
\text { DSULAD }\end{array}$ & $\begin{array}{l}\text { (IAY) } \\
\text { OCOOL. }\end{array}$ & OCOOLS & $\begin{array}{l}S F F \\
E F F T\end{array}$ & $\begin{array}{l}\text { IFACY } \\
\text { FFFO }\end{array}$ & 000 & DCT PHWT & $\begin{array}{l}\text { SUF SULT } \\
\text { PCTAOCE }\end{array}$ & PrTHTS & SOLCOOI & \\
\hline $\bar{\delta}$ & 1 & 75 & प्रद & of . 5 & $1-1.4$ & व.? & 210.5 & $83 . n$ & .141 & .256 & .035 & 0.000 & .770 & 0.000 & .394 & \\
\hline$A$ & ?. & 75 & 1009.4 & 3.5 & j.e & 0.0 & $0 . n$ & $n \cdot n$ & - 0104 & .045 & 0.1100 & $0.0 n n$ & .0 .000 & $0.0 \cap 0$ & 0.000 & \\
\hline 8 & 3 & 15 & 1135.5 & $2 \div 4$ & $\geq 1.9$ & $n \cdot n$ & $0 \cdot n$ & $n \cdot n$ &. .174 &.$? 4 ?$ & n. noin & $0 . n n n$ & n. $\cap n ?$ & 0.000 & $0.00 n$ & \\
\hline s & 4 & 75 & 11315.9 & -744.4 & $5 i=.2$ & 479.7 & 252.5 & 261.4 & .176 & .2 .33 & .565 & $0.0 n n$ & .099 & 1.000 & .994 & \\
\hline B & 5 & 75 & $9 \div 5.0$ & 40.9 & 235.0 & 151.7 & $2+2.4$ & 133.4 & .179 & $.27 n$ & .037 & $0 . n$ in & .417 & n. nno & .537 & \\
\hline B & $\theta$ & 75 & $0 \in 5.7$ & $=5 \leq .1$ & $1=? .2$ & $63 . ?$ & 24.56 & -69.7 & -. $\cap 97$. & .234 & $\ldots .056$ & D.con & .169 & .107 & .247 & \\
\hline 8 & 7 & 75 & 973.4 & 53.7 & 102.0 & $23 . \overline{3}$ & 149.3 & 10.5 & .134 & . 296 & .557 & $0.00 n$ & . คA7 & 0.000 & .131 & \\
\hline $\begin{array}{l}\mathrm{s} \\
\mathrm{a}\end{array}$ & $\stackrel{8}{4}$ & $\begin{array}{l}75 \\
75 \\
\end{array}$ & $\begin{array}{l}1190.5 \\
1141.5\end{array}$ & $\begin{array}{r}153.1 \\
-164 . ?\end{array}$ & $\begin{array}{l}137.0 \\
375.0\end{array}$ & $\begin{array}{r}62.4 \\
2 B 1.5\end{array}$ & $\begin{array}{r}109.0 \\
234.1\end{array}$ & $\begin{array}{r}47.3 \\
133.7\end{array}$ & $\begin{array}{r}1171 \\
.120\end{array}$ & $\begin{array}{r}.236 \\
.237\end{array}$ & $\begin{array}{l}.520 \\
.527\end{array}$ & $\begin{array}{l}0.00 n \\
0.00 n\end{array}$ & $\begin{array}{r}202 \\
.510\end{array}$ & $\begin{array}{l}0.000 \\
0.000\end{array}$ & $\begin{array}{r}.436 \\
.464\end{array}$ & \\
\hline 8 & 10 & $75 \pi$ & luve.? & -9.4 & 9.7 & ก. & 145.8 & $0 . n$ & 0.000 & 0.000. &.$=45$ & $0.0 \mathrm{nn}$ & $0.0 n n$ & 0.000 & $0.00 n$ & \\
\hline$B$ & 11 & $75 \%$ & 1003.4 & 36.5 & $1 i 7.3$ & 79.4 & $463 . n$ & $5 n .6$ & .147 & .702 & .524 & $0.0 \cap n$ &.$n 9 n$ & 0.000 & .100 & \\
\hline$\tilde{b}$ & 12 & 73 & 981.4 & -15.4 & 79.9 & $n \cdot n$ & 404.7 & $n \cdot n$ & .074 & _? ?14 & .491 & $0.0 \cap n$ & 0.000 & 0.000 & 0.008 & \\
\hline 5 & 13 & 15 & $4 \overline{3} 5.4$ & $-7=.5$ & 73.3 & 0.0 & 0.0 & 0.0 & 0.000 & $0.0 n 0$ & 0.000 & 0.000 & $0 . \operatorname{nn}$ & 0.000 & $0.00 n$ & \\
\hline 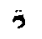 & 14 & 75 & द्र44.6 & -17.7 & 31.3 & 0.0 & 0.0 & 0.0 & .065 & .224 & 0.000 & $0.0 n n$ & 0.000 & $n .000$ & 0.000 & \\
\hline A & 12 & 15 & $9 \div 0.0$ & $10^{4} 2^{5}$ & 39.9 & $n, ?$ & $0 . n$ & $n \cdot n$ & .125 & . $2 A R$ & $0 . n 0 ?$ & $n .80 n$ & $0.00 n$ & 0.000. & $0.00 n_{-}$ & \\
\hline 5 & 10 & 15 & 1051.0 & 105.4 & 79.5 & $n .0$ & $0 . n$ & $n . n$ & .133 & $.25 a$ & 0.1000 & $0.0 \cap n$ & $0 . \cap \cap n$ & 0.000 & $0.00 \mathrm{r}$ & $\omega$ \\
\hline 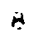 & 17 & 75 & 1090.4 & 2.4 & $1: 4.1$ & a. $n$ & 0.0 & $n . n$ & .075 & . ?.20 & n.ron & $0.0 n n$ & $0 . n n 0$ & 0.000 & 0.000 & - \\
\hline 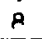 & is & 75 & 1050.9 & - & 333.7 & $215 . ?$ & 410.1 & 123.9 & .145 & .343 & .519 & $0.00 n$ & . ?7? & $n . \cap \cap 0$ & $-30 ?$ & \\
\hline $\bar{H}$ & 15 & 73 & dichon & -3.5 & 15\%.7 & $44 . n$ & 411.1 & 31.7 & $.11)^{4}$ & .258 & $=10$ & 0.000 & nica & 0.000 & .076 & \\
\hline 3 & 20 & 75 & 1605.3 & 4.1 & 47.6 & 1.? & $503 . ?$ & . $A$ & .053 & .1 .37 & $.5 \cap 4$ & $n . n \cap n$ & . n01 & $0.0 \cap 0$ &.$n \cap 2$ & \\
\hline 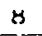 & 21 & 75 & 11.21 .9 & $1=.5$ & $244 . ?$ & 179.4 & 288.1 & $\ln k . ?$ & $.17 n$ & .20 .3 & $.5 \cap R$ & $0.0 \cap n$ & .214 & 0.000 & $.36^{\circ}$ & 二 \\
\hline$m$ & ¿2? & 75 & li112.0 & -25.6 & $2: \div 7$ & 132.4 & 222.1 & 99.7 & .132 & .223 & .401 & $0.0 n \pi$ & .750 & 0.000 & .445 & \\
\hline 8 & 25 & 75 & 1:8.7 & $1<n .0$ & 120.6 & $n .0$ & 0.0 & $a . n$ &.$J B A$ & . 3?? & 0.000 & 0.000 & $0.0 \cap n$ & $n .0,0$ & $0.00 n$ & \\
\hline 8 & 74 & 15 & 1154.5 & $2 ? .2$ & ioi. 1 & 21.7 & 5.3 & 5.3 & .117 & .254 & .745 & $0.0 n n$ & $1 . n n n$ & 0.000 & $1 . n \cap n$ & \\
\hline$\overline{4}$ & 5 & 75 & T丁यू. & $=64.7$ & 213.1 & 175.4 & 57.3 & 57.3 & .145 & .234 & .327 & $0.00 n$ & $1 . \operatorname{nnn}$ & 0.000 & $1.0 n n$ & \\
\hline 8 & 76 & 75 & 1981.4 & -73.3 & -75.7 & 363.7 & 242.1 & 197.9 &.$? 27$ & .312 & $.55 n$ & $0.0 n n$ & .226 & 0.000 & .919 & \\
\hline A & 27 & 75 & $101 \leq r$ & $-7 \div .3$ & 23.7 & 150.4 & 47.3 .5 & 11.3 .1 & .126 & $-.23 \mathrm{~K}$ & $.58 h$ & $0.00 n$ & -197 & 0.000 & $-230^{\circ}$ & \\
\hline 9 & a & 75 & $4=3 . ?$ & 11. & <5 5.1 & 207.7 & $56.3 . ?$ & 150.9 & .194 & .313 & .571 & $0.00 n$ & .211 & 0.000 & .260 & \\
\hline a & 29 & 75 & $1(1) 14.7$ & 3.0 & $=51.7$ & 327.6 & 514.8 & 195.8 & .244 & .373 & $.53 n$ & $0.00 n$ & .237 & 0.000 & $.38 n$ & \\
\hline$A$ & 30 & 75 & $1118:=?$ & -1.2 & $-5-1.1$ & 347.5 & 418.4 & $183 . n$ & .254 & $.33 ?$ & .497 & $0.00 \mathrm{C}$ & .413 & n.coo & $.43 ?$ & \\
\hline 5 & 31 & 75 & $11=2.6$ & 7.7 & $\therefore 5 \times .1$ & 364.9 & 499.3 & 187.7 & .246 & .378 & .508 & 0.000 & .371 & 0.000 & $.37 A$ & \\
\hline & TOT & $T \Delta L$ & 31457.4 & 135.9 & $E \leq 52.7$ & 3783.5 & 7135.6 & $2 ? 41 \cdot 5$ & & & & & & & & \\
\hline & $a_{1}$ & EAT & jत30.0 & 4.4 & 207.3 & $1 \geq ? 00$ & $230 . ?$ & 72.3 & $.15 n$ & .272 & .535 & 0.000 & .793 & .617 & $.31 \mathrm{a}$ & \\
\hline
\end{tabular}


baILY TOTAL HEAT HATES (HJJOAY)

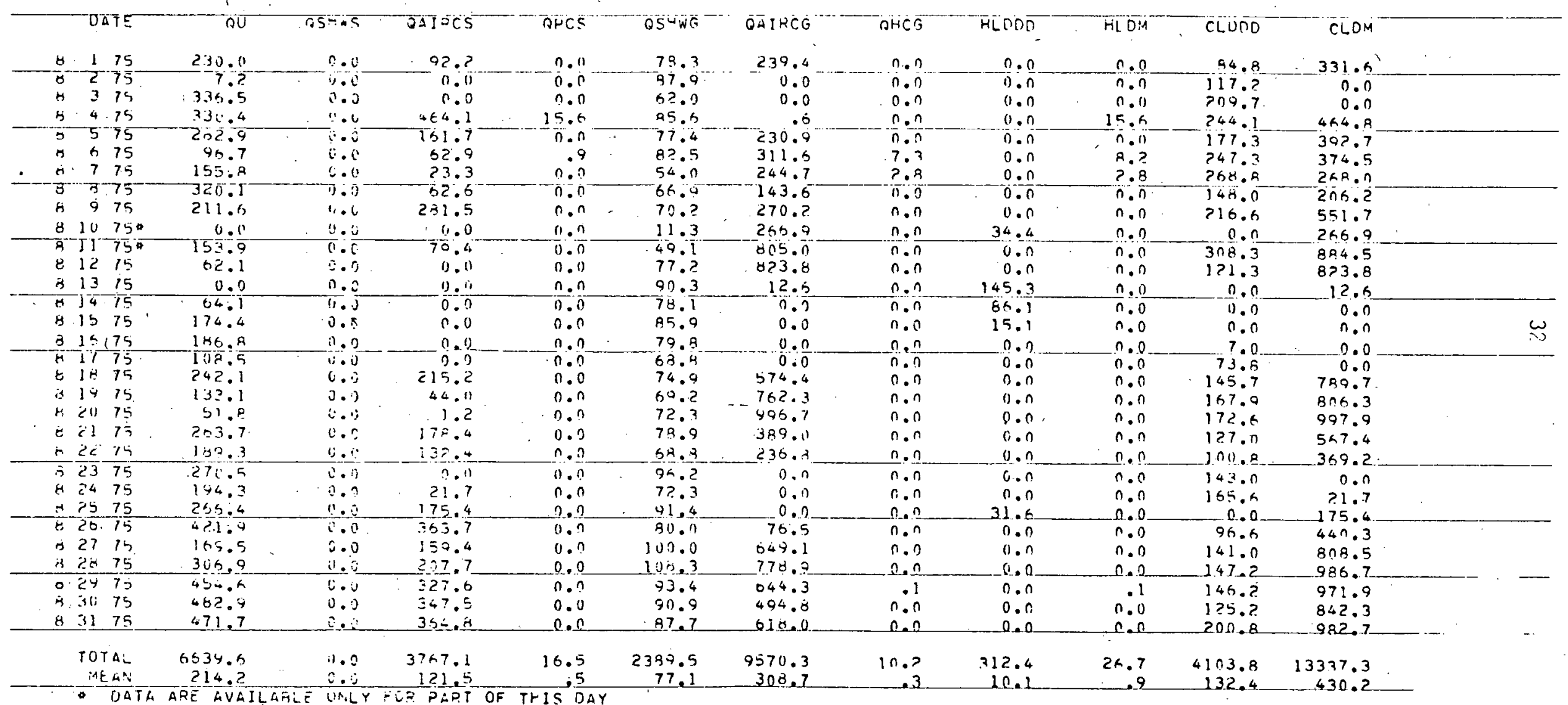

Figure 15 (con't) 


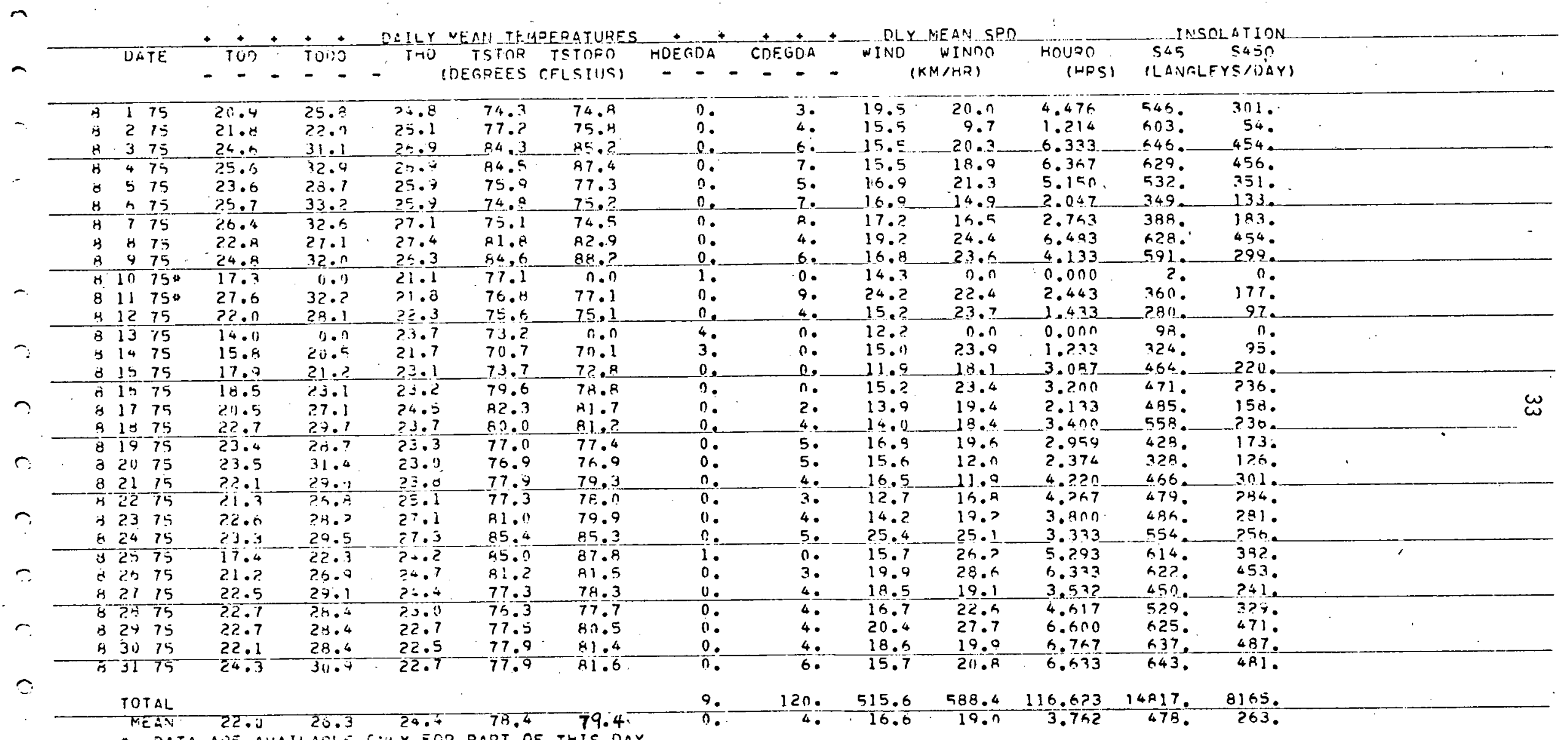

- data are available ciey for part of this ony

Figure $15\left(\operatorname{con}^{\prime} t\right)$ 
SYSTEM THERMAL'PERFORMANCE

DAILY, MONTHLY, AND ANNUAL VALUES OF THE MEAN DAILY HEAT FLOWS

\section{Total Energy Required}

Tables 4 through 15 show the daily values of the total energy required for the heating and cooling loads, the supplemental energy required (natural gas is used in the auxiliary boiler), the solar energy incident on the collector (insolation measured in the plane of the collectors), solar energy collected, and solar energy delivered to load. These data were obtained during the period 1 September 1974 to 31 August 1975. Table 16 shows the monthly and annual averages of daily heat rates for the same period.

The total energy required is determined from the flow rates and temperature differences across the particular load, of the heat input by either solar or auxiliary. Supplemental energy required and solar energy delivered from storage to load is obtained by the same method, but where the position of automatic valve, V2, determines whether solar or auxiliary is actually furnishing the energy to load. Incident solar energy data were obtained from a solar pyranometer and solar energy collected and stored is based on the collector loop flow rate and temperature difference across the collector/storage heat exchanger.

\section{Supplemental Energy Required}

Supplemental energy requirements were met by an auxiliary, natural gas-fired, hot water boiler. For heating the auxiliary boiler was used whenever the solar portion of the heating system was deemed unable to meet 
Table 4. (September 1974) DAILY TOTAL HEAT RATES (MJ/DAY)

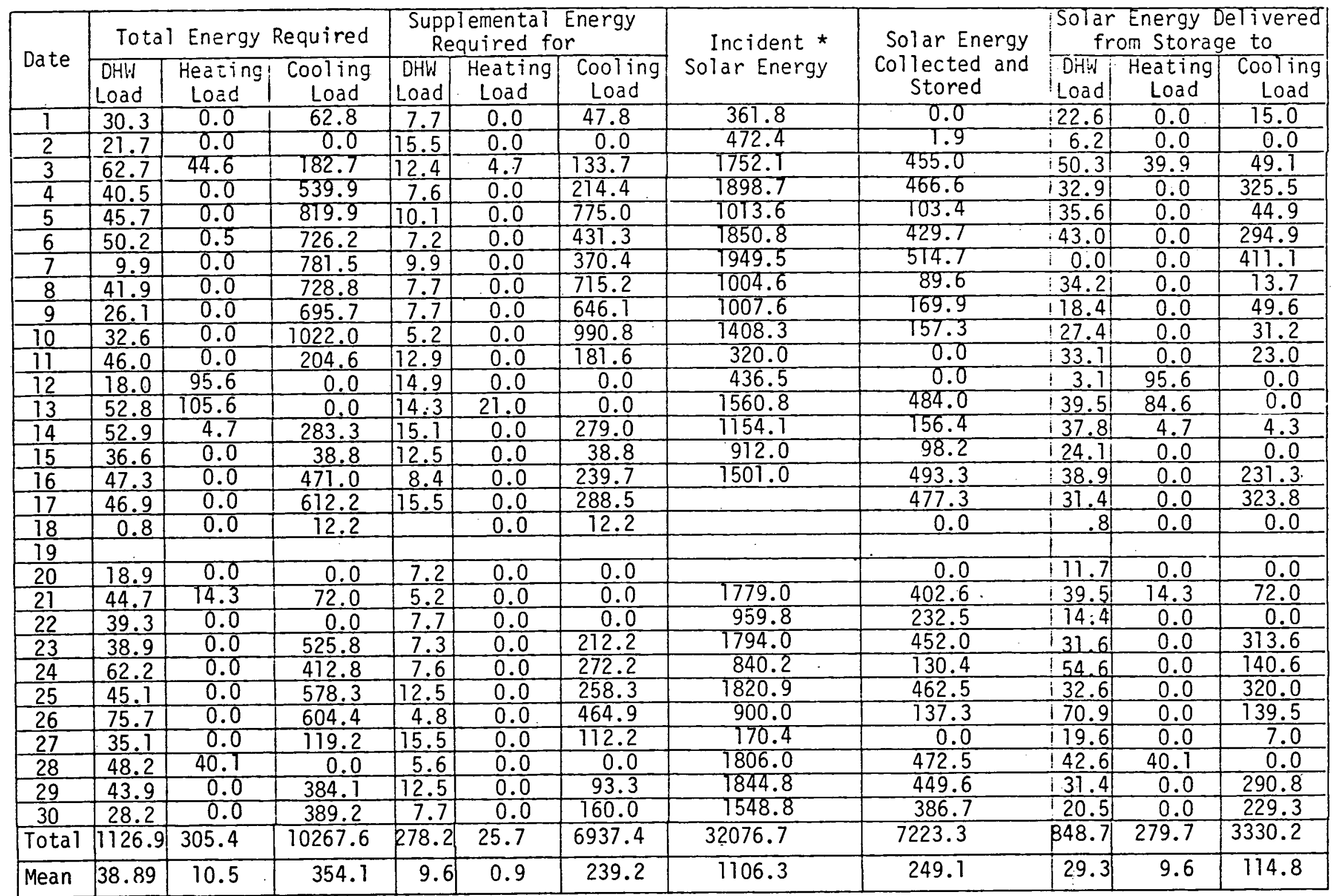

*0n a 71.3 square meter solar collector surface 
Table 5. (October 1974) DAILY TOTAL HEAT REATES (MJ/DAY)

\begin{tabular}{|c|c|c|c|c|c|c|c|c|c|c|c|}
\hline \multirow{2}{*}{ Date } & \multicolumn{3}{|c|}{ Total Energy Required } & \multicolumn{3}{|c|}{$\begin{array}{l}\text { Supplemental Energy } \\
\text { Required for }\end{array}$} & \multirow{2}{*}{$\begin{array}{l}\text { Incident * } \\
\text { Solar Energy }\end{array}$} & \multirow{2}{*}{$\begin{array}{l}\text { Solar Energy } \\
\text { Collected aris } \\
\text { Stored }\end{array}$} & \multicolumn{3}{|c|}{$\begin{array}{l}\text { Solar Energy Delivered } \\
\text { from Storage to }\end{array}$} \\
\hline & $\begin{array}{l}\text { DHW } \\
\text { Load } \\
\end{array}$ & $\begin{array}{l}\text { Heating } \\
\text { Load }\end{array}$ & $\begin{array}{l}\text { Cooling } \\
\text { Load }\end{array}$ & $\begin{array}{c}\text { DHW } \\
\text { Load } \\
\end{array}$ & $\begin{array}{l}\text { Heating } \\
\text { Load }\end{array}$ & $\begin{array}{l}\text { Cooling } \\
\text { Load }\end{array}$ & & & Corisi & $\begin{array}{c}\text { Heating } \\
\text { Load }\end{array}$ & $\begin{array}{c}\text { Cooling } \\
\text { Load }\end{array}$ \\
\hline 1 & 33.1 & 0.0 & 635.5 & 13.9 & 0.0 & 351.8 & 1731.2 & 419.6 & 119.21 & 0.0 & 283.7 \\
\hline 2 & 97.7 & 0.0 & 104.6 & 50.6 & 0.0 & 66.6 & 1761.1 & 341.4 & 47.11 & 0.0 & 38.0 \\
\hline 3 & 141.8 & 0.0 & 265.1 & 56.0 & 0.0 & 7.2 & 1372.4 & 281.3 & 85.8 & 0.0 & 257.9 \\
\hline 4 & 33.5 & 0.0 & 529.6 & 7.8 & 0.0 & 326.9 & 1297.7 & 304.6 & 25.7 & 0.0 & 202.7 \\
\hline 5 & 52.2 & 0.0 & 622.0 & 14.1 & 0.0 & 620.5 & $1172: 1$ & 97.7 & 38.1 & 0.0 & 1.5 \\
\hline 6 & 45.9 & 0.0 & 188.2 & 8.4 & 0.0 & 217.6 & 1405.3 & 287.1 & 37.5 & 0.0 & 60.6 \\
\hline 7 & 137.9 & 0.0 & 248.3 & 8.4 & 0.0 & 128.8 & 212.3 & 38.7 & 723.5 & 0.0 & 119.4 \\
\hline 8 & 60.2 & 0.0 & 694.6 & 0.0 & 0.0 & 444.4 & 1447.2 & 427.2 & 50.2 & 0.0 & 250.2 \\
\hline 9 & 36.1 & 0.0 & 805.7 & 7.2 & 0.0 & 527.9 & 1336.5 & 422.1 & 28.9 & 0.0 & 277.7 \\
\hline 10 & 23.2 & 0.0 & 376.5 & 5.2 & 0.0 & 316.5 & 290.0 & 25.9 & 78.0 & 0.0 & 0.0 \\
\hline 11 & 27.9 & 0.0 & 466.9 & 7.7 & 0.0 & 466.9 & 251.2 & 0.0 & 20.2 & 0.0 & 0.0 \\
\hline 12 & 26.8 & 0.0 & 214.5 & 8.0 & 0.0 & 274.5 & 128.6 & 0.0 & 18.8 & 0.0 & 0.0 \\
\hline 13 & 47.7 & 0.0 & 0.0 & 14.6 & 0.0 & 0.0 & 1770.1 & 443.1 & 33.1 & 0.0 & 0.0 \\
\hline 14 & 9.9 & 0.0 & 0.0 & 4.8 & 0.0 & 0.0 & 927.0 & 155.6 & 5.1 & 0.0 & 0.0 \\
\hline 15 & & & & & & & & & $i$ & & \\
\hline 16 & 58.4 & 0.0 & 181.6 & 12.4 & 0.0 & 181.4 & 517.3 & 124.7 & 46.0 & 0.0 & 0.2 \\
\hline 17 & 39.4 & 0.0 & 30.1 & 0.0 & 0.0 & 0.0 & 1728.2 & 430.4 & 39.4 & 0.0 & 30.1 \\
\hline 18 & & & & & & & & & 1 & & \\
\hline 19 & & & & & & & & & I & & \\
\hline 20 & & & & & & & & & $!$ & & \\
\hline 21 & 18.7 & 0.0 & 297.4 & 0.0 & 0.0 & 226.9 & 164.5 & 3.4 & 178.7 & 0.0 & 64.4 \\
\hline 22 & 14.0 & 0.0 & 74.2 & 0.0 & 0.0 & 56.6 & 26.9 & 0.0 & 14.0 & 0.0 & 17.7 \\
\hline 23 & 30.4 & 0.0 & 0.0 & 7.6 & 0.0 & 0.0 & 92.7 & 0.0 & $\frac{17.0}{22.8}$ & 0.0 & 0.0 \\
\hline 24 & 44.9 & 0.0 & 0.0 & 0.0 & 0.0 & 0.0 & 62.8 & 0.0 & 44.9 & 0.0 & 0.0 \\
\hline 25 & 711.5 & 0.0 & 9.8 & 7.2 & 0.0 & 9.8 & 26.9 & 115.1 & 104.3 & 0.0 & 0.0 \\
\hline 26 & 88.3 & 0.0 & 0.0 & 11.9 & 0.0 & 0.0 & 1471.1 & 317.4 & 76.4 & 0.0 & 0.0 \\
\hline 27 & 63.2 & 0.0 & 0.0 & 12.8 & 0.0 & 0.0 & 1518.9 & 311.4 & 50.4 & 0.0 & 0.0 \\
\hline 28 & 83.0 & 0.0 & 0.0 & 6.6 & 0.0 & 0.0 & 1318.6 & 269.2 & 76.4 & 0.0 & 0.0 \\
\hline 29 & 46.3 & 8.9 & 0.0 & 6.6 & 0.0 & 0.0 & 406.6 & -0.5 & 32.7 & 8.9 & 0.0 \\
\hline 30 & 33.6 & 126.6 & 0.0 & 8.3 & 7.0 & 0.0 & 242.2 & 0.0 & 25.3 & 119.6 & 0.0 \\
\hline 31 & 18.3 & 148.5 & 0.0 & 13.2 & 0.0 & 0.0 & 248.2 & 19.5 & 5.1 & 148.5 & 0.0 \\
\hline Total & 1418.2 & 284.1 & 5678.4 & 293.5 & 7.0 & 4074.1 & 22933.3 & 4834.7 & il24.7 & 277.1 & 1604.2 \\
\hline Mean & 52.6 & 10.6 & 210.4 & 10.9 & 0.3 & 150.9 & 849.2 & 179.1 & 41.7 & 10.3 & 59.4 \\
\hline
\end{tabular}


Table 6 (November 1974) DAILY TOTAL HEAT RATES (MJ/DAY)

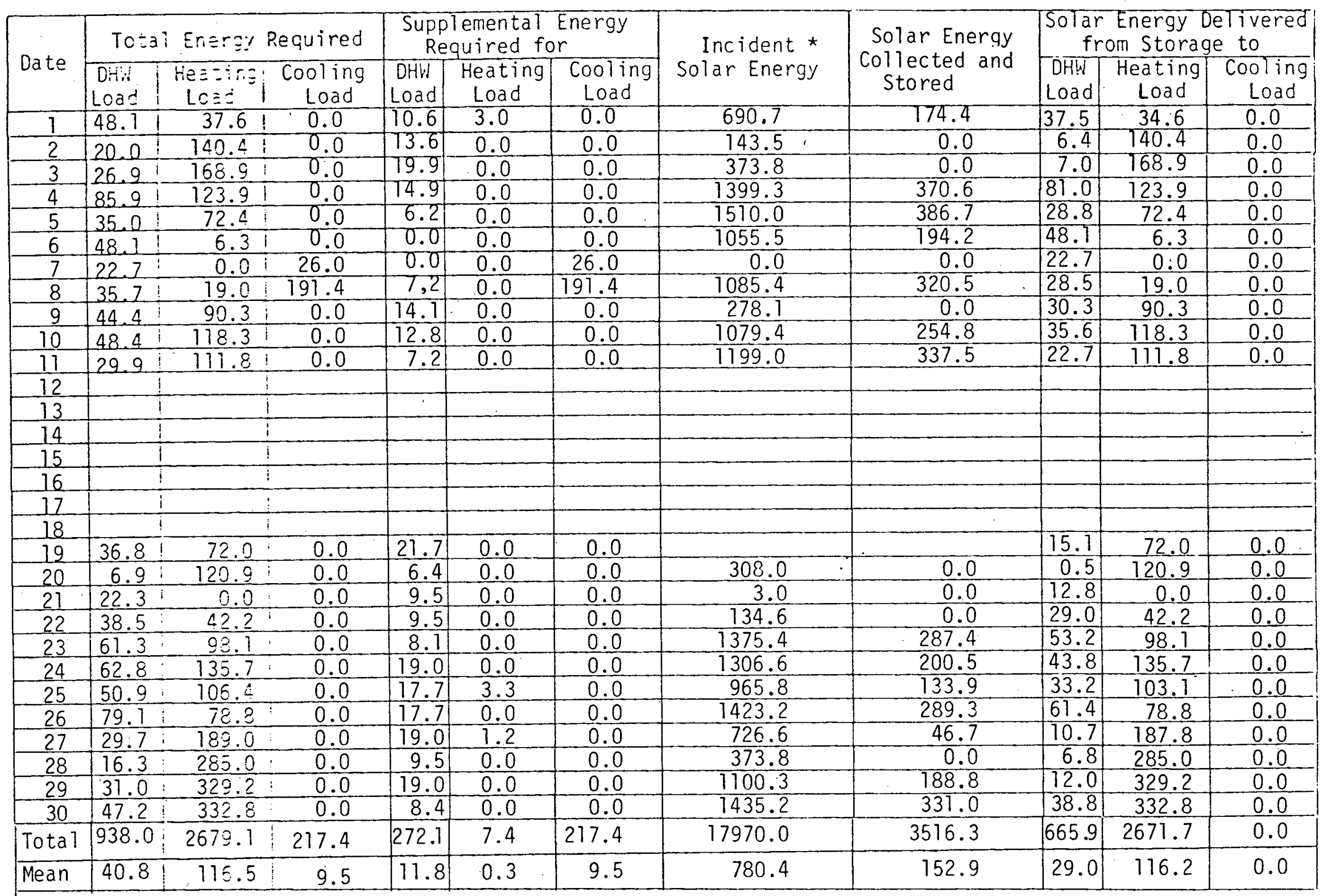

$\star 0$ a 71.3 scuare meter solar collector surface 
Table 7. (December 1974) DAILY TOTAL HEAT RATES (MJ/DAY)

\begin{tabular}{|c|c|c|c|c|c|c|c|c|c|c|c|}
\hline \multirow{2}{*}{ Da te } & \multicolumn{3}{|c|}{ Total Er:Erg: Required } & \multicolumn{3}{|c|}{$\begin{array}{l}\text { Supplemental Energy } \\
\text { Required for }\end{array}$} & \multirow{2}{*}{$\begin{array}{l}\text { Incident * } \\
\text { Solar Energy }\end{array}$} & \multirow{2}{*}{$\begin{array}{l}\text { Solar Energy } \\
\text { Collected and } \\
\text { Stored }\end{array}$} & \multicolumn{3}{|c|}{$\begin{array}{l}\text { Solar Energy Delivered } \\
\text { from Storage to }\end{array}$} \\
\hline & $\begin{array}{l}\text { DH!i! } \\
\text { Load }\end{array}$ & $\begin{array}{c}\text { He }=-i r g \\
\text { LC } \equiv=\end{array}$ & $\begin{array}{c}\text { Cooling } \\
\text { Load }\end{array}$ & \begin{tabular}{|l|} 
DHW \\
Load \\
\end{tabular} & $\begin{array}{c}\text { Heating } \\
\text { Load }\end{array}$ & $\begin{array}{c}\text { Cooling } \\
\text { Load }\end{array}$ & & & $\begin{array}{l}\text { DHW } \\
\text { Load } \\
\end{array}$ & $\begin{array}{l}\text { Heating } \\
\text { Load }\end{array}$ & $\begin{array}{l}\text { Cooling } \\
\text { Load }\end{array}$ \\
\hline$T$ & 67.6 & 234.2 & 0.0 & 17.7 & 0.0 & 0.0 & 1441.2 & 377.5 & 49.9 & 234.2 & 0.0 \\
\hline 2 & 52.9 & 125.8 & 0.0 & 9.5 & 0.0 & 0.0 & 1408.2 & 306.6 & 43.4 & 125.8 & 0.0 \\
\hline 3 & 44.0 & 119.0 & 0.0 & 8.1 & 0.0 & 0.0 & 1213.9 & 182.6 & 35.9 & 119.0 & 0.0 \\
\hline 4 & 46.0 & 3.6 & 0.0 & 10.9 & 0.0 & 0.0 & 1465.1 & 277.2 & 35.1 & 3.6 & 0.0 \\
\hline 5 & 35.0 & 67.2 & 0.0 & 20.4 & 2.5 & 0.0 & 687.7 & 59.7 & 14.6 & 64.7 & 0.0 \\
\hline 6 & 70.6 & 98.7 & 0.0 & 9.5 & 0.0 & 0.0 & 1396.3 & 332.1 & 61.1 & 98.7. & 0.0 \\
\hline$T$ & 26.2 & 282.2 & 0.0 & 19.0 & 0.0 & 0.0 & 251.2 & 0.0 & 7.2 & 282.2 & 0.0 \\
\hline 8 & 44.4 & 220.3 & 0.0 & 8.1 & 0.0 & 0.0 & 1447.2 & 449.0 & 36.3 & 220.3 & 0.0 \\
\hline 9 & 68.9 & 130.6 & 0.0 & 19.0 & 0.0 & 0.0 & 1420.3 & 403.4 & 49.9 & 130.6 & 0.0 \\
\hline 70 & 9.5 & 86.1 & 0.0 & 0.0 & 0.0 & 0.0 & 143.5 & 0.0 & 9.5 & 86.1 & 0.0 \\
\hline 12 & & $\frac{1}{4}$ & & & & & & & & & \\
\hline 13 & & 7 & & & & & & & & & \\
\hline 14 & & I & & & & & & & & & \\
\hline 15 & & & & & & & & & & & \\
\hline 76 & & I & & & & & & & & & \\
\hline$\frac{17}{18}$ & & $\frac{1}{1}$ & & & & & & & & & \\
\hline 19 & 19.6 & 91.3 & 0.0 & 8.1 & 1.1 & 0.0 & 128.6 & 6.4 & 11.5 & 90.2 & 0.0 \\
\hline 20 & 16.9 & 252.0 & 0.0 & 12.8 & 72.4 & 0.0 & 290.0 & 3.6 & 4.1 & 179.6 & 0.0 \\
\hline 21 & 35.7 & 325.8 & 0.0 & 19.0 & 287.0 & 0.0 & 454.5 & 42.4 & 16.7 & 38.8 & 0.0 \\
\hline 22 & 56.8 & 355.5 & 0.0 & 19.0 & 127.2 & 0.0 & 1453.1 & 457.3 & 37.8 & 228.3 & 0.0 \\
\hline 23 & 19.2 & 416.8 & 0.0 & 17.7 & 232.2 & 0.0 & 158.5 & 0.0 & 1.5 & 184.6 & 0.0 \\
\hline 24 & $37.4 !$ & 357.5 & 0.0 & 9.5 & 198.6 & 0.0 & 1223.0 & 266.2 & 21.9 & 159.0 & 0.0 \\
\hline 25 & 53.9 & 381.3 & 0.0 & 17.7 & 169.7 & 0.0 & 1483.0 & 442.4 & 36.2 & 211.6 & 0.0 \\
\hline 26 & 27.8 & 347.7 & 0.0 & 19.0 & 60.1 & 0.0 & 538.2 & 77.2 & 2.8 & 287.6 & 0.0 \\
\hline 27 & 50.0 & 253.7 & 0.0 & 8.1 & 143.8 & 0.0 & 1240.9 & 407.2 & 47.9 & 109.9 & 0.0 \\
\hline 28 & 50.0 & 231.2 & 0.0 & 19.0 & 0.0 & 0.0 & 1172.1 & 349.2 & 37.0 & 231.2 & 0.0 \\
\hline 29 & 32.0 & 294.5 & 0.0 & 19.0 & 0.0 & 0.0 & 950.8 & 265.0 & 13.0 & 294.5 & 0.0 \\
\hline 30 & 54.8 & 237.7 & 0.0 & 14.9 & 0.0 & 0.0 & 938.9 & 252.8 & 39.9 & 237.7 & 0.0 \\
\hline 37 & 40.8 & 271.1 & 11.2 & 17.7 & 0.0 & 11.2 & 1109.3 & 280.8 & 23.1 & 271.1 & 0.0 \\
\hline Total & 948.2 & 5184.7 & 11.2 & 323.9 & 1294.7 & 11.2 & 22015.4 & 5232.5 & 624.3 & 3889.4 & 0.0 \\
\hline Mean & 41.2 & 225.6 & 0.5 & $\mid 74.1$ & 56.3 & 0.5 & 956.8 & 227.5 & $|27.1|$ & 169.1 & 0.0 \\
\hline
\end{tabular}

${ }^{*}$ On a 71.3 square meter solar collector surface 
Táble 8. (January 1975) DAILY TOTAL HEAT RATES (MJ/DAY)

\begin{tabular}{|c|c|c|c|c|c|c|c|c|c|c|c|}
\hline \multirow[b]{2}{*}{ Date } & \multicolumn{3}{|c|}{ Total Er. $=$ rgy Required } & \multicolumn{3}{|c|}{$\begin{array}{l}\text { Supplemental Energy } \\
\text { Required for }\end{array}$} & \multirow{2}{*}{$\begin{array}{l}\text { Incident * } \\
\text { Solar Energy }\end{array}$} & \multirow{2}{*}{$\begin{array}{l}\text { Solar Energy } \\
\text { Collected and } \\
\text { and Stored }\end{array}$} & \multicolumn{3}{|c|}{$\begin{array}{l}\text { Solar Energy Delivered } \\
\text { from Storage to }\end{array}$} \\
\hline & \begin{tabular}{|l|} 
DHid \\
Load
\end{tabular} & $\begin{array}{c}\text { He } \equiv=\dot{i n g} \\
\text { Lozd }\end{array}$ & $\begin{array}{c}\text { Cooting } \\
\text { Load }\end{array}$ & $\begin{array}{l}\text { DHW } \\
\text { Load } \\
\end{array}$ & $\begin{array}{l}\text { Heating } \\
\text { Load }\end{array}$ & $\begin{array}{c}\text { Cooling } \\
\text { Load }\end{array}$ & & & \begin{tabular}{|c|} 
D $\overline{H W}$ \\
Load
\end{tabular} & $\begin{array}{l}\text { Heating } \\
\text { Load }\end{array}$ & $\begin{array}{l}\text { Cooling } \\
\text { Load }\end{array}$ \\
\hline 7 & 39.0 & 265.6 & 0.0 & 74.4 & 0.0 & 0.0 & 1447.2 & 418.6 & 24.6 & 265.6 & 0.0 \\
\hline 2 & 14.4 & 356.6 & 0.0 & 74.4 & 54.3 & 0.0 & 278.1 & 0.0 & 0.0 & 302.3 & 0.0 \\
\hline 3 & 42.8 & 313.2 & 0.0 & 13.4 & 184.2 & 0.0 & 1375.4 & 409.3 & 29.4 & 129.0 & 0.0 \\
\hline 4 & 26.7 & 234.7 & 0.0 & 6.2 & 0.0 & 0.0 & 1250.0 & 359.2 & 20.5 & 234.7 & 0.0 \\
\hline 5 & 36.4 & 253.4 & 0.0 & 13.4 & 0.0 & 0.0 & 1336.5 & 383.6 & 23.0 & 253.4 & 0.0 \\
\hline 6 & 55.3 & 193.9 & 0.0 & 14.4 & 0.0 & 0.0 & 1399.3 & 387.6 & 40.9 & 193.9 & 0.0 \\
\hline 7 & 84.1 & 192.4 & 0.0 & 18.9 & 0.8 & 0.0 & 822.3 & 127.7 & 65.2 & 191.6 & 0.0 \\
\hline 8 & 38.0 & 222.9 & 0.0 & 25.3 & 0.0 & 0.0 & 639.9 & 137.9 & 12.7 & 222.9 & 0.0 \\
\hline 9 & 30.6 & 221.9 & 0.0 & 20.7 & 0.0 & 0.0 & 941.9 & 196.1 & 9.9 & 221.9 & 0.0 \\
\hline 10 & 24.9 & 385.0 & 0.0 & 16.6 & 757.5 & 0.0 & 750.5 & 184.7 & 8.3 & 227.5 & 0.0 \\
\hline 11 & 24.3 & 710.2 & 0.0 & 21.4 & 632.8 & 0.0 & 275.3 & 0.0 & 2.9 & 77.4 & 0.0 \\
\hline 12 & 41.5 & 510.8 & 0.0 & 23.6 & 302.3 & 0.0 & 1058.5 & 219.1 & 17.9 & 208.5 & 0.0 \\
\hline 13 & 54.5 & 301.4 & 0.0 & 46.1 & 208.6 & 0.0 & 705.6 & 120.5 & 8.4 & 92.8 & 0.0 \\
\hline 14 & 53.7 & 117.7 & 0.0 & 14.5 & 54.1 & 0.0 & 1178.1 & 352.4 & 38.6 & 63.6 & 0.0 \\
\hline 15 & 43.5 & 218.6 & 0.0 & 32.5 & 0.0 & 0.0 & 735.5 & 136.9 & 71.0 & 218.6 & 0.0 \\
\hline 16 & 43.11 & 271.6 & 0.0 & 38.7 & 18.9 & 0.0 & 651.8 & 125.2 & 5.0 & 192.7 & 0.0 \\
\hline 17 & 68.8 & 141.8 & 0.0 & 30.8 & 60.7 & 0.0 & 1019.6 & 299.5 & 38.0 & 81.1 & 0.0 \\
\hline 18 & 48.7 & 124.8 & 0.0 & 26.7 & 0.0 & 0.0 & 1228.9 & 324.5 & 22.6 & 124.8 & 0.0 \\
\hline 19 & 44.9 & 197.3 & 0.0 & 20.3 & 0.0 & 0.0 & 1459.1 & 384.1 & 24.6 & 197.3 & 0.0 \\
\hline 20 & 57.31 & 140.1 & 0.0 & 23.1 & 0.0 & 0.0 & 1213.9 & 264.3 & 34.2 & 140.7 & 0.0 \\
\hline 27 & 39.7 & 204.3 & 0.0 & 19.0 & 0.0 & 0.0 & 99.27 & 216.0 & 20.7 & 204.3 & 0.0 \\
\hline 22 & 39.2 & 203.4 & 0.0 & 29.8 & 0.0 & 0.0 & 1480.1 & 373.3 & 9.4 & 203.4 & 0.0 \\
\hline 23 & 46.3 & 150.8 & 0.0 & 28.3 & 3.4 & 0.0 & 843.2 & 156.5 & 18.0 & 147.4 & 0.0 \\
\hline 24 & 14.7 & 132.2 & 0.0 & 13.9 & 0.0 & 0.0 & 648.8 & 179.2 & 0.8 & 132.2 & 0.0 \\
\hline 25 & 1 & 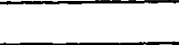 & $\vdots$ & & & & & & & & \\
\hline 26 & & 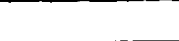 & $!$ & & & & & & & & \\
\hline 27 & 35.3 & 292.7 & 0.0 & 35.3 & 208.7 & 0.0 & 137.6 & 0.0 & 0.0 & 84.0 & 0.0 \\
\hline 28 & 77.3 & 379.8 & 0.0 & 53.2 & 308.0 & 0.0 & 849.2 & 184.0 & 24.1 & 71.8 & 0.0 \\
\hline 29 & 98.9 & 189.4 & 0.0 & 43.8 & 0.0 & 0.0 & 1512.9 & 436.8 & 55.1 & 189.4 & 0.0 \\
\hline 30 & 53.8 & 230.2 & 0.0 & 53.8 & 0.0 & 0.0 & 1231.9 & 393.3 & 0.0 & 230.2 & 0.0 \\
\hline 31 & 85.0 & 225.0 & 0.0 & 39.7 & 99.3 & 0.0 & 1512.9 & 833.8 & 45.3 & 125.7 & 0.0 \\
\hline Total & 1362.4 & 7322.0 & 0.0 & 751.2 & 2293.8 & 0.0 & 28919.3 & 7604.3 & 611.2 & 5028.2 & 0.0 \\
\hline Mean & 47.0 & 252.6 & 0.0 & 25.9 & 79.1 & 0.0 & 998.7 & 262.2 & 21.1 & 173.4 & 0.0 \\
\hline
\end{tabular}

* On a 71.3 square meter solar collector surface 
Table 9. (February 1975) DAILY TOTAL HEAT RATES (MJ/DAY)

\begin{tabular}{|c|c|c|c|c|c|c|c|c|c|c|c|}
\hline \multirow{2}{*}{ Date } & \multicolumn{3}{|c|}{ Totai EnErgy Required } & \multicolumn{3}{|c|}{$\begin{array}{l}\text { Supplemental Energy } \\
\text { Required for }\end{array}$} & \multirow{2}{*}{$\begin{array}{l}\text { Incident * } \\
\text { Solar Energy }\end{array}$} & \multirow{2}{*}{$\begin{array}{l}\text { Solar Energy } \\
\text { Collected and } \\
\text { Stored }\end{array}$} & \multicolumn{3}{|c|}{$\begin{array}{l}\text { Solar Energy Delivered } \\
\text { from Storage to }\end{array}$} \\
\hline & DHin & $\begin{array}{c}\text { He }=-i n: \\
\text { LC } \equiv d\end{array}$ & $\begin{array}{l}\text { Cooling } \\
\text { Load }\end{array}$ & $\begin{array}{l}\text { OHW } \\
\text { LOad }\end{array}$ & $\begin{array}{l}\text { Heating } \\
\text { Load }\end{array}$ & $\begin{array}{l}\text { Cooling } \\
\text { Load }\end{array}$ & & & $\begin{array}{l}\text { DHW } \\
\text { Load } \\
\end{array}$ & $\begin{array}{l}\text { Heating } \\
\text { Load }\end{array}$ & $\begin{array}{l}\text { Cooling } \\
\text { Load }\end{array}$ \\
\hline$T$ & 86.11 & 256.21 & 0.0 & 18.3 & 172.4 & 0.0 & 1602.6 & 545.0 & 67.8 & 83.8 & 0.0 \\
\hline 2 & 54.3 & 220.9 & 0.0 & 9.8 & 0.0 & 0.0 & 1617.6 & 546.6 & 54.5 & 220.9 & 0.0 \\
\hline 3 & 50.71 & 126.9 & 0.0 & 27.7 & 0.0 & 0.0 & 1784.0 & 346.8 & 29.0 & 126.9 & 0.0 \\
\hline 4 & 88.0 & 187.4 & 0.0 & 48.2 & 0.0 & 0.0 & 710.6 & 0.0 & 39.8 & 187.9 & 0.0 \\
\hline 5. & 98.3 & 363.4 & 0.0 & 89.5 & 174.4 & 0.0 & 559.1 & 3.0 & 8.8 & 189.0 & 0.0 \\
\hline 6 & 87.6 & 389.5 & 0.0 & 43.0 & 371.5 & 0.0 & 1644.5 & 443.1 & 44.6 & 18.0 & 0.0 \\
\hline 7 & 88.6 & 247.4 & 0.0 & 26.0 & 164.9 & 0.0 & 1477.1 & 351.4 & 62.6 & 82.5 & 0.0 \\
\hline 8 & 37.4 & 425.2 & 0.0 & 37.4 & 0.0 & 0.0 & 379.7 & 0.0 & 0.0 & 425.2 & 0.0 \\
\hline 9 & 91.5 & 473.6 & 0.0 & 74.8 & 313.5 & 0.0 & 717.6 & 113.4 & 16.7 & 100.7 & 0.0 \\
\hline 10 & 64.2 & 151.4 & 0.0 & 62.9 & 101.5 & 0.0 & 777.4 & 167.6 & 1.3 & 49.9 & 0.0 \\
\hline 11 & 118.1 & 139.3 & 0.0 & 48,4 & 0.0 & 0.0 & 1728.2 & 581.3 & 69.7 & 139.3 & $0.0^{\prime}$ \\
\hline 12 & 95.4 & 128.1 & 0.0 & 29.4 & 16.7 & 0.0 & 1357.5 & 332.4 & 66.0 & 111.4 & 0.0 \\
\hline 13 & 88.31 & $99: 3$ & 0.0 & 32.9 & 0.0 & 0.0 & 756.5 & 128.6 & 55.4 & 99.3 & 0.0 \\
\hline 14 & 56.71 & 124.6 & 0.0 & 38.2 & 0.0 & 0.0 & 278.1 & 0.0 & 18.5 & 124.6 & 0.0 \\
\hline 15 & $91.0 !$ & 294.9 & 0.0 & 72.8 & 1.4 & 0.0 & 1106.3 & 84.4 & 18.2 & 293.5 & 0.0 \\
\hline 16 & 82.31 & 313.0 & 0.0 & 52.6 & 38.2 & 0.0 & 1722.2 & 426.8 & 29.7 & 274.8 & 0.0 \\
\hline 17 & 130.7 & 211.4 & 0.0 & 99.9 & 0.0 & 0.0 & 1258.8 & 321.7 & 30.8 & 271.4 & 0.0 \\
\hline 18 & 144.91 & 273.4 & 0.0 & 70.6 & 0.0 & 0.0 & 1856.8 & 573.9 & 74.3 & 273.4 & 0.0 \\
\hline 19 & $714.7 !$ & 298.7 & 0.0 & 50.4 & 0.0 & 0.0 & 1704.3 & 498.1 & 64.3 & 298.7 & 0.0 \\
\hline 20 & 76.21 & 271.3 & 0.0 & 29.4 & 4.4 & 0.0 & 1357.5 & 303.1 & 46.8 & 266.9 & 0.0 \\
\hline 21 & $74.0 !$ & 380.6 & 0.0 & 33.3 & 0.0 & 0.0 & 1608.6 & 441.9 & 40.7 & 380.6 & 0.0 \\
\hline 22 & 95.61 & 378.6 & 0.0 & 26.8 & 0.0 & 0.0 & 1776.1 & 527.2 & 68.8 & 378.6 & 0.0 \\
\hline 23 & 45.7 & 327.0 & 0.0 & 29.9 & 0.0 & 0.0 & 1085.4 & 286.0 & 15.8 & 321.0 & 0.0 \\
\hline 24 & $80.8:$ & 67.6 & 0.0 & 6.2 & 0.0 & 0.0 & 1297.7 & 326.5 & 74.6 & 67.6 & 0.0 \\
\hline 25 & 98.0 & 202.9 & 0.0 & 78.9 & 0.0 & 0.0 & 1770.1 & 394.4 & 79.7 & 202.9 & 0.0 \\
\hline 26 & 110.6 & 179.1 & 0.0 & 32.4 & 0.0 & 0.0 & 1770.1 & $4 \longdiv { 4 . 6 }$ & 78.2 & 179.1 & 0.0 \\
\hline 27 & 128.4 & 91.0 & 0.0 & 18.3 & 1.7 & 0.0 & 1719.3 & 365,0 & 170.1 & 89.3 & 0.0 \\
\hline 28 & 90.5 & 76.7 & 0.0 & 20.1 & 0.0 & 0.0 & 1477.1 & 256.9 & 70.4 & 76.6 & 0.0 \\
\hline Total & 2478.1 & 6633.3 & 0.0 & 7141.6 & 1360.5 & 0.0 & 35703.6 & 8774.5 & 7336.5 & 5272.8 & 0.0 \\
\hline Mean & 88.5 & 236.9 & 0.0 & 40.8 & 48.6 & 0.0 & 1273.7 & 313.4 & 4.7 .7 & 188.3 & 0.0 \\
\hline
\end{tabular}

*On a 71.3 square meter solar collector surface 
Table 10. (March 1975) DAILY TOTAL HEAT RATES (MJ/DAY)

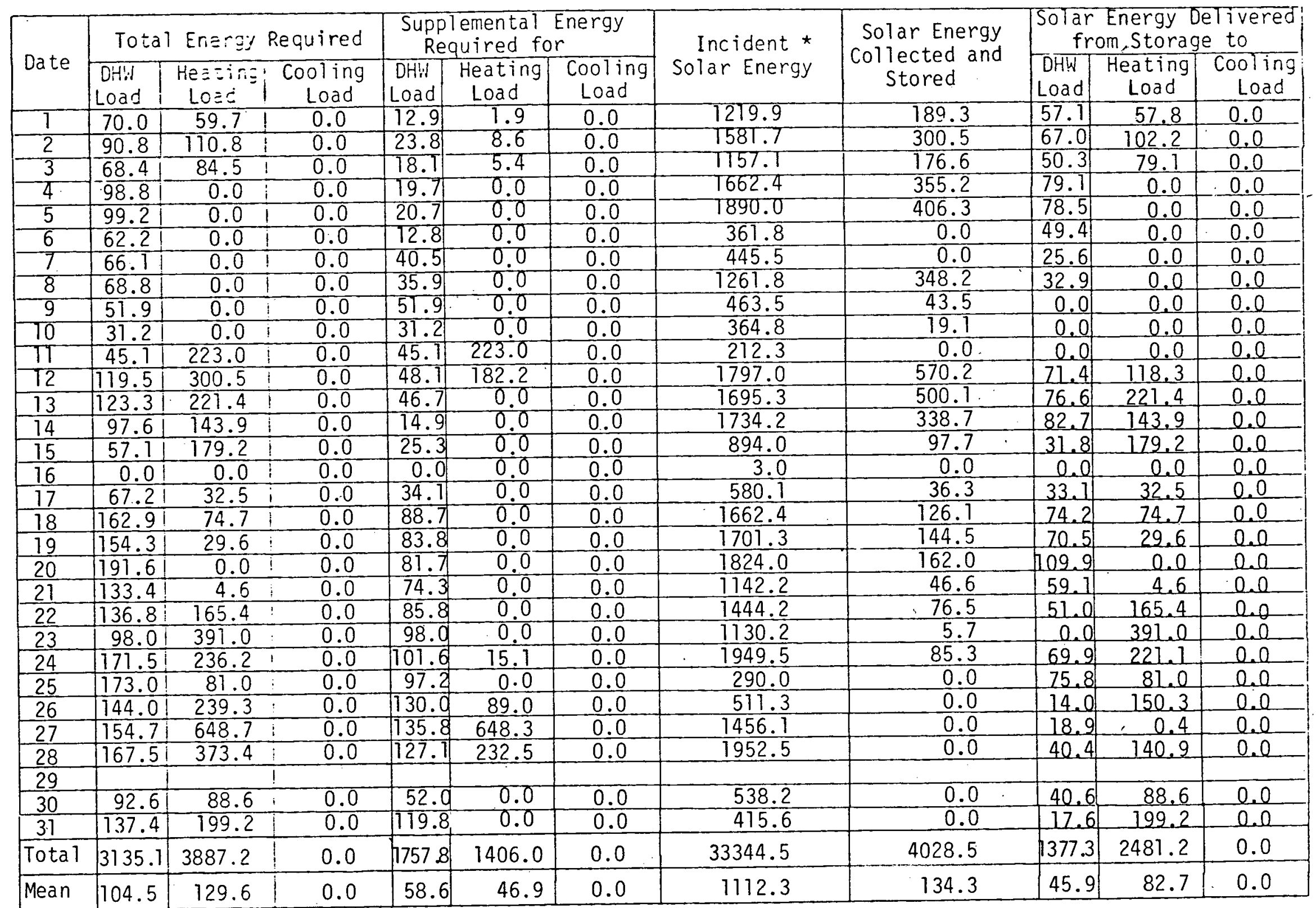

*On a 71.3 square meter solar collector surface 
Table 11. (April 1975) DAILY TOTAL HEAT RATES (MJ/DAY)

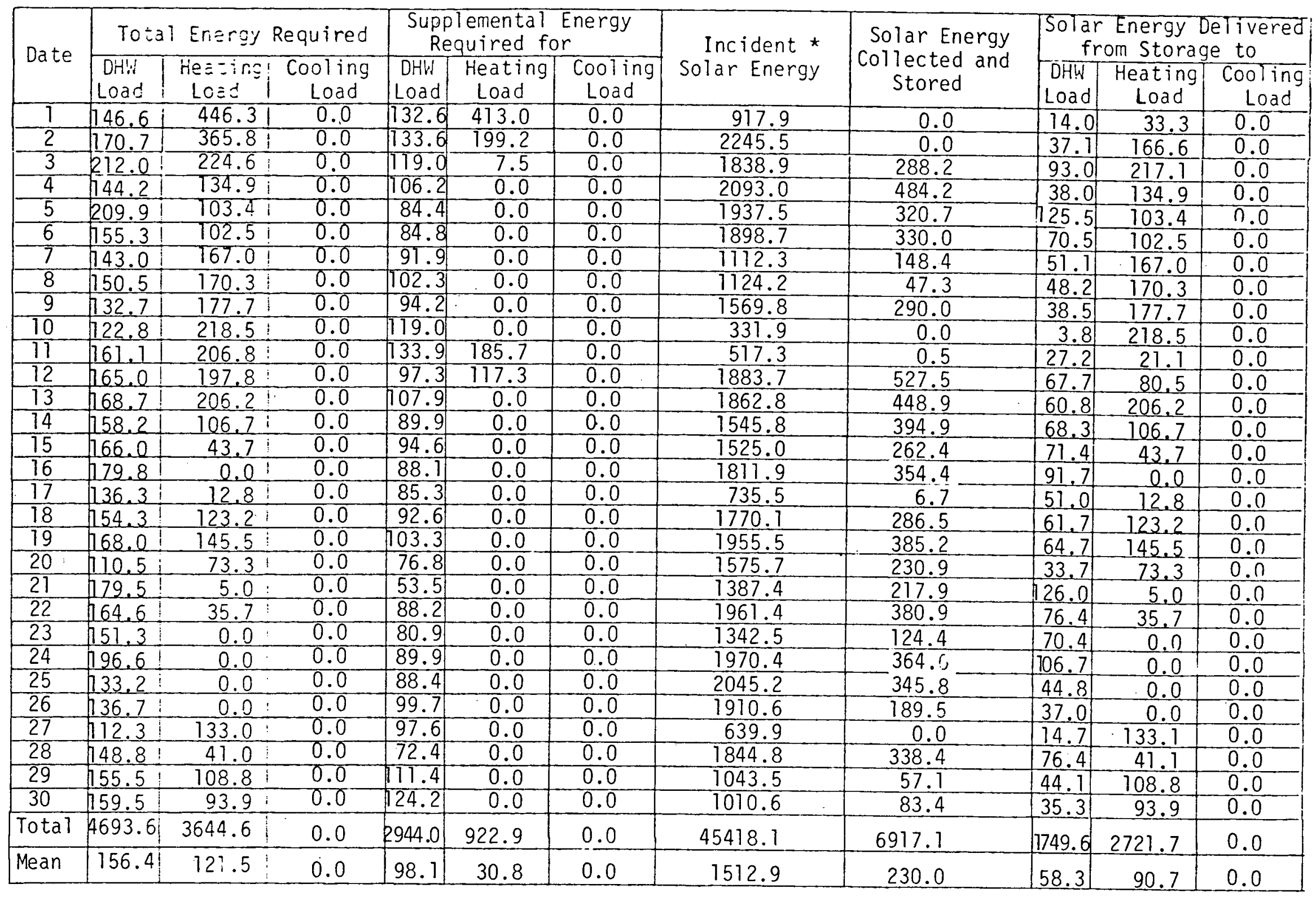

*On a 71.3 square meter solar collector surface 


\begin{tabular}{|c|c|c|c|c|c|c|c|c|c|c|c|}
\hline \multirow{2}{*}{ Date } & \multicolumn{3}{|c|}{ Total Energy Required } & \multicolumn{3}{|c|}{$\begin{array}{l}\text { Supplemental Energy } \\
\text { Required for }\end{array}$} & \multirow{2}{*}{$\begin{array}{l}\text { Incident * } \\
\text { Solar Energy }\end{array}$} & \multirow{2}{*}{$\begin{array}{l}\text { Solar Energy } \\
\text { Collected and } \\
\text { Stored }\end{array}$} & \multicolumn{3}{|c|}{$\begin{array}{l}\text { Solar Energy, Delivered } \\
\text { from Storage to }\end{array}$} \\
\hline & \begin{tabular}{|l|}
$\mathrm{DHW}$ \\
Load
\end{tabular} & 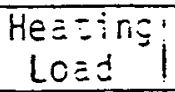 & $\begin{array}{l}\text { Cooling } \\
\text { Load }\end{array}$ & \begin{tabular}{|l|} 
DHW \\
Load \\
\end{tabular} & $\begin{array}{l}\text { Heating } \\
\text { Load }\end{array}$ & $\begin{array}{l}\text { Cooling } \\
\text { Load }\end{array}$ & & & \begin{tabular}{|c|} 
DHW \\
Load
\end{tabular} & $\begin{array}{l}\text { Heating } \\
\text { Load }\end{array}$ & $\begin{array}{l}\text { Cooling } \\
\text { Load }\end{array}$ \\
\hline 1 & 180.7 & 68.2 & 0.0 & 104.1 & 0.0 & 0.0 & 1444.2 & 327.0 & 76.0 & 68.2 & 0.0 \\
\hline 2 & 151.7 & 72.3 & 0.0 & 97.8 & 0.0 & 0.0 & 1578.9 & 254.9 & 53.9 & 12.3 & 0.0 \\
\hline$\frac{2}{3}$ & 279.0 & 7.0 & 0.0 & 99.2 & 0.0 & 0.0 & 1817.9 & 347.5 & 119.8 & 7.0 & 0.0 \\
\hline 4 & 215.2 & 30.0 & 0.0 & 700.9 & 0.0 & 0.0 & 1800.0 & 300.6 & 174.3 & 0.0 & 0.0 \\
\hline 5 & 231.4 & 10.0 & 0.0 & 82.3 & 0.0 & 0.0 & 1234.9 & 160.9 & 49.1 & 0.0 & 0.0 \\
\hline 6 & 191.3 & 78.6 & 0.0 & 100.7 & 22.7 & 0.0 & 1441.2 & 164.6 & 90.6 & 57.9 & 0.0 \\
\hline 7 & 181.2 & 55.2 & 0.0 & 712.6 & 0.0 & 0.0 & 744.4 & 10.6 & 68.6 & 55.2 & 0.0 \\
\hline 8 & 134.0 & 37.7 & 0.0 & 771.2 & 0.0 & 0.0 & 1118.3 & 146.7 & 22.8 & 37.7 & 0.0 \\
\hline 9 & 168.1 & 6.1 & 0.0 & 74.8 & 0.0 & 0.0 & 1417.3 & 368.0 & 93.3 & 6.1 & 0.0 \\
\hline 10 & 90.5 & 0.0 & 0.0 & 58.8 & 0.0 & 0.0 & 1007.6 & 97.6 & 31.7 & 0.0 & 0.0 \\
\hline 11 & 124.4 & 0.0 & 0.0 & 92.3 & 0.0 & 0.0 & 1629.6 & 309.9 & 32.1 & $0 ; 0$ & 0.0 \\
\hline 12 & 120.1 & 8.0 & 0.0 & 94.8 & 0.0 & 0.0 & 891.0 & 63.7 & 25.3 & 8.0 & 0.0 \\
\hline 13 & 166.8 & 4.4 & 0.0 & 00.2 & 0.0 & 0.0 & 1806.0 & 354.9 & 66.6 & 4.4 & 0.0 \\
\hline 14 & 125.5 & 11.8 & 0.0 & 85.9 & 0.0 & 0.0 & 1892.7 & 379.7 & 39.6 & 11.8 & 0.0 \\
\hline 15 & 91.5 & 0.0 & 0.0 & 74.9 & 0.0 & 0.0 & 1324.6 & 225.0 & 16.6 & 0.0 & 0.0 \\
\hline 16 & 1724.6 & 0.0 & 0.0 & 82.5 & 0.0 & 0.0 & 1372.4 & 162.2 & 42.1 & 0.0 & 0.0 \\
\hline 17 & 107.1 & 0.0 & 0.0 & 80.6 & 0.0 & 0.0 & 1258.8 & 172.1 & 26.5 & 0.0 & 0.0 \\
\hline 18 & 119.8 & 0.0 & 0.0 & 84.7 & 0.0 & 0.0 & 1216.9 & 215.7 & 35.7 & 0.0 & 0.0 \\
\hline 19 & 144.7 & 0.0 & 0.0 & 81.3 & 0.0 & 0.0 & 1417.1 & 184.0 & 63.4 & 0.0 & 0.0 \\
\hline 20 & 146.6 & 0.0 & 0.0 & 81.4 & 0.0 & 0.0 & 367.8 & 0.0 & 65.2 & 0.0 & 0.0 \\
\hline 27 & 149.5 & 31.2 & 0.0 & 86.2 & 0.0 & 0.0 & 236.2 & 0.0 & 63.3 & 31.2 & 0.0 \\
\hline 22 & 145.8 & 3.3 & 0.0 & 76.0 & 0.0 & 0.0 & 421.6 & 30.8 & 69.8 & 3.3 & 0.0 \\
\hline 23 & 275.5 & 23.3 & 0.0 & 133.9 & 0.0 & 0.0 & 1058.5 & 178.8 & 141.6 & 23.3 & 0.0 \\
\hline 24 & 220.9 & 1.2 & 0.0 & 172.1 & 0.0 & 0.0 & 1877.7 & 433.5 & 108.8 & 1.2 & 0.0 \\
\hline 25 & 205.1 & 0.0 & 0.0 & 97.6 & 0.0 & 0.0 & 183.4 & 13.0 & 107.5 & 0.0 & 0.0 \\
\hline 26 & 193.5 & 30.3 & 0.0 & 90.6 & 0.0 & 0.0 & 1899.7 & 348.2 & 102.9 & 30.3 & 0.0 \\
\hline 27 & 173.2 & 0.0 & 0.0 & 90.8 & 0.0 & 0.0 & 1184.0 & 0.0 & 82.4 & 0.0 & 0.0 \\
\hline 28 & 183.6 & 0.0 & 0.0 & 710.3 & 0.0 & 0.0 & 212.3 & 0.0 & 73.3 & 0.0 & 0.0 \\
\hline 29 & 148.1 & 58.8 & 0.0 & 719.3 & 0.0 & 0.0 & 503.3 & 0.0 & 28.8 & 58.8 & 0.0 \\
\hline 30 & 177.7 & 34.8 & 0.0 & 115.3 & 0.0 & 0.0 & 1614.6 & 346.3 & 62.4 & 34.8 & 0.0 \\
\hline 31 & 88.7 & 0.0 & 0.0 & 62.2 & 0.0 & 0.0 & 1124.2 & 188.7 & 26.5 & $0 \Omega$ & 0.0 \\
\hline Total & 4995.1 & 474.2 & 0.0 & 2895.4 & 22.7 & 0.0 & 37725.0 & 5664.75 & 2099.7 & 451.5 & 0.0 \\
\hline Mean & 161.1 & 15.3 & 0.0 & 93.4 & 0.7 & 0.0 & 1216.9 & 182.7 & 67.7 & 14.6 & 0.0 \\
\hline
\end{tabular}

*On a 71.3 square meter solar collector surface 
Table 13. (June 1975) DAILY TOTAL HEAT RATES (MJ/DAY)

\begin{tabular}{|c|c|c|c|c|c|c|c|c|c|c|c|}
\hline \multirow{2}{*}{ Date } & \multicolumn{3}{|c|}{ Total En $=$ rgy Required } & \multicolumn{3}{|c|}{$\begin{array}{l}\text { Supplemental Energy } \\
\text { Required for }\end{array}$} & \multirow{2}{*}{$\begin{array}{l}\text { Incident * } \\
\text { Solar Energy }\end{array}$} & \multirow{2}{*}{$\begin{array}{c}\text { Solar Energy } \\
\text { Collected and } \\
\text { Stored }\end{array}$} & \multicolumn{3}{|c|}{$\begin{array}{l}\text { Solar Energy Delivered } \\
\text { from Storage to }\end{array}$} \\
\hline & \begin{tabular}{|l|} 
DHin \\
Load
\end{tabular} & $\begin{array}{l}H E \equiv=:-B \\
\text { LCES }\end{array}$ & $\begin{array}{c}\text { Cooling } \\
\text { Load }\end{array}$ & $\begin{array}{c}\text { DHW } \\
\text { Load } \\
\end{array}$ & $\begin{array}{l}\text { Heating } \\
\text { Load }\end{array}$ & $\begin{array}{c}\text { Cooling } \\
\text { Load }\end{array}$ & & & $\begin{array}{c}\text { OHW } \\
\text { Load } \\
\end{array}$ & $\begin{array}{l}\text { Heating } \\
\text { Load }\end{array}$ & $\begin{array}{c}\text { Cooling } \\
\text { Load }\end{array}$ \\
\hline 1 & 166.0 & 5.9 & 10.0 & 101.7 & 0.0 & 0.0 & 1859.8 & 360.0 & \begin{tabular}{|l|}
64.3 \\
\end{tabular} & 5.9 & 0.0 \\
\hline 2 & 174.8 & 0.0 & $1 \quad 0.0$ & 89.9 & 0.0 & 0.0 & 1797.0 & 237.0 & 84.9 & 0.0 & 0.0 \\
\hline 3 & 83.7 & 0.0 & 349.7 & 79.5 & 0.0 & 349.7 & 1399.3 & 118.2 & 04.2 & 0.0 & 0.0 \\
\hline 4 & $\overline{68.7}$ & 0.0 & 0.0 & 84.4 & 0.0 & 0.0 & 1689.4 & 295.4 & 84.3 & 0.0 & 0.0 \\
\hline 5 & 155.9 & 0.0 & 0.0 & 79.6 & 0.0 & 0.0 & 1916.6 & 303.2 & 76.3 & 0.0 & 0.0 \\
\hline 6 & $737.2 !$ & 0.0 & 1502.8 & 74.2 & 0.0 & 358.0 & 1566.8 & 180.4 & 57.0 & 0.0 & 144.9 \\
\hline 7 & 160.9 & 1.3 & 589.6 & 94.2 & 1.3 & 555.9 & 1208.0 & 187.2 & 66.7 & 0.0 & 33.7 \\
\hline 8 & 149.4 & 1.3 & 335.7 & 89.1 & 1.3 & 333.9 & 795.3 & 35.6 & 60.3 & 0.0 & 1.8 \\
\hline 9 & 127.01 & 0.0 & $\div \quad 0.0$ & 88.5 & 0.0 & 0.0 & 849.2 & 64.3 & 38.5 & 0.0 & 0.0 \\
\hline 70 & 137.01 & 15.5 & $\quad 28.2$ & 104.8 & 0.0 & 28.2 & 693.7 & 14.1 & 32.2 & 15.5 & 0.0 \\
\hline 11 & 149.6 & 12.4 & 1234.1 & 96.5 & 0.0 & 234.1 & 1770.1 & 378.4 & 53.7 & 12.4 & 0.0 \\
\hline 12 & $102.8 !$ & 5.2 & 437.5 & 86.7 & 1.7 & 431.5 & 1387.4 & 244.6 & 76.1 & 4.7 & 0.0 \\
\hline 13 & 714.7 & $\overline{0.0}$ & 419.1 & 83.5 & 0.0 & 372.5 & 1629.6 & 229.9 & 37.2 & 0.0 & 46.5 \\
\hline 14 & 0177.01 & 0.0 & 605.6 & 99.4 & -2.7 & 605.6 & 1220.0 & 74.5 & 17.6 & 0.0 & 0.0 \\
\hline 15 & $95.1 !$ & 0.0 & 348.5 & 69.6 & 0.0 & 249.7 & 1806.0 & 329.3 & 25.5 & 0.0 & 98.7 \\
\hline 76 & 45.01 & 1.0 & 353.1 & 38.4 & 0.0 & 353.1 & 633.8 & 4.0 & 6.6 & 1.0 & 0.0 \\
\hline 77 & $107.1:$ & 0.0 & 276.0 & 48.0 & 0.0 & 275.0 & 879.3 & 65.7 & 53.1 & 0.0 & 1.1 \\
\hline 18 & 95.9 & 0.0 & 315.4 & 70.6 & 0.0 & 375.4 & 780.4 & $7^{\prime} 01.2$ & 25.3 & 0.0 & 0.0 \\
\hline 19 & 138.2 & 0.0 & 253.1 & 64.1 & 0.0 & 253.1 & 1510.0 & 223.7 & 74.7 & 0.0 & 0.0 \\
\hline 20 & 150.71 & 0.0 & 529.2 & 85.7 & 0.0 & 426.6 & 1629.6 & 302.5 & 65.0 & 0.0 & 102.7 \\
\hline 21 & 149.3 & 0.0 & $: 380.0$ & 87.11 & 0.0 & 378.3 & 1136.0 & 113.9 & 68.2 & 0.0 & 1.7 \\
\hline 22 & 136.31 & 0.0 & 182.4 & 76.2 & 0.0 & 182.4 & 1387.4 & 200.1 & 60.1 & 0.0 & 0.0 \\
\hline 23 & 77.8 & 0.0 & .396 .1 & 64.5 & 0.0 & 158.5 & 1818.0 & 327.3 & 13.3 & 0.0 & 237.5 \\
\hline 24 & 129.7 & 0.0 & 613.2 & 53.9 & 0.0 & 477.3 & 7527.9 & 282.4 & 75.8 & 0.0 & 135.9 \\
\hline 25 & $138.7:$ & 0.0 & 627.8 & 64.9 & 0.0 & 479.7 & 1507.0 & 281.8 & 73.8 & 0.0 & 148.1 \\
\hline 26 & & & 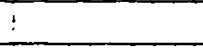 & & & & & & & & \\
\hline 27 & & & $\vdots$ & & & & & & & & \\
\hline 28 & 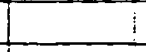 & & 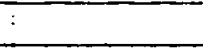 & & & & & & & & \\
\hline 29 & $i$ & & & & & & & & & & \\
\hline 30 & & & & & & & & & & & \\
\hline Total & 3295.9 & 39.9 & 7771.2 & 1968.7 & 1.0 & .6818 .5 & 34373.0 & 4968.6 & 1327.2 & 38.9 & 952.6 \\
\hline Mean & 1131.8 & 1.6 & 310.8 & 78.7 & 0.0 & 272.7 & 1375.4 & 198.7 & 53.1 & 1.6 & 38.1 \\
\hline
\end{tabular}

*On a 71.3 square meter solar collector surface 
Table 14. (July 1975) DAILY TOTAL HEAT RATES (MJ/DAY)

\begin{tabular}{|c|c|c|c|c|c|c|c|c|c|c|c|}
\hline \multirow{2}{*}{ Date } & \multicolumn{3}{|c|}{ Totai Eriaryy Required } & \multicolumn{3}{|c|}{$\begin{array}{l}\text { Supplemental Energy } \\
\text { Required for }\end{array}$} & \multirow{2}{*}{$\begin{array}{l}\text { Incident * } \\
\text { Solar Energy }\end{array}$} & \multirow{2}{*}{$\begin{array}{l}\text { Solar Energy } \\
\text { Collected and } \\
\text { Stored }\end{array}$} & \multicolumn{3}{|c|}{$\begin{array}{l}\text { Solar Energy Delivered } \\
\text { from Storage to }\end{array}$} \\
\hline & \begin{tabular}{|l|} 
DHin \\
Load \\
\end{tabular} & $\begin{array}{l}\text { HeE-in: } \\
\text { LCE: }\end{array}$ & 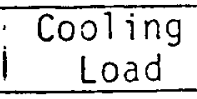 & $\begin{array}{l}\text { DHW } \\
\text { Load }\end{array}$ & $\begin{array}{l}\text { Heating } \\
\text { Load }\end{array}$ & $\begin{array}{l}\text { Cooling } \\
\text { Load }\end{array}$ & & & $\begin{array}{c}\text { DHW } \\
\text { Load } \\
\end{array}$ & $\begin{array}{l}\text { Heating } \\
\text { Load }\end{array}$ & $\begin{array}{l}\text { Cooling } \\
\text { Load }\end{array}$ \\
\hline 1 & 61.51 & 0.0 & 653.0 & 61.5 & 0.0 & 495.4 & 1892.7 & 396.0 & 0.0 & 0.0 & 157.6 \\
\hline 2 & $65.4 !$ & 0.0 & 681.1 & 65.4 & 0.0 & 454.8 & 1665.4 & 342.3 & 0.0 & 0.0 & 226.3 \\
\hline 3 & 63.1 & 2.5 & 526.6 & 63.1 & 2.5 & 354.3 & 1405.3 & 304.2 & 0.0 & 0.0 & 172.3 \\
\hline 4 & 68.7 & 2.7 & 729.9 & 68.7 & 4.7 & 486.6 & 1811.9 & 371.5 & 0.0 & 0.0 & 243.3 \\
\hline 5 & 79.11 & 1.5 & i. 452.7 & 79.1 & 1.5 & 295.9 & 1784.0 & 237.5 & 0.0 & 0.0 & 156.9 \\
\hline$\frac{6}{7}$ & & & $i$ & & & & & & & & \\
\hline 7 & 55.5 & 0.0 & 663.2 & 55.5 & 0.0 & 585.9 & 1142.2 & 152.9 & 0.0 & 0.0 & 77.3 \\
\hline 8 & 88.4 & 0.0 & 823.0 & 88.4 & 0.0 & 606.2 & 1764.1 & 335.7 & 0.0 & 0.0 & 216.7 \\
\hline 9 & 76.2 & 0.0 & 1200.6 & 76.2 & 0.0 & 222.2 & 1097.3 & 137.4 & 0.0 & 0.0 & -21.4 \\
\hline 10 & 73.5 & 0.0 & 587.8 & 73.5 & 0.0 . & 474.6 & 1623.6 & 282.2 & 0.0 & 0.0 & 173.1 \\
\hline 11 & 85.9 & 0.0 & 340.5 & 85.9 & 0.0 & 774.6 & 1725.2 & 339.7 & 0.0 & 0.0 & 225.9 \\
\hline 12 & 76.5 & 0.0 & 107.0 & 76.5 & 0.0 & 0.0 & 1826.9 & 320.0 & 0.0 & 0.0 & 107.0 \\
\hline 13 & 69.2 & 0.0 & 273.1 & 69.2 & 0.0 & 0.0 & 1773.1 & 310.4 & 0.0 & 0.0 & 273.1 \\
\hline 14 & 69.4 & 0.0 & 393.1 & 69.4 & 0.0 & 316.7 & 7016.6 & 63.2 & 0.0 & 0.0 & 77.1 \\
\hline 15 & 97.4 & 0.0 & $1 \quad 345.9$ & 97.4 & 0.0 & 124.3 & 1336.5 & 208.3 & 0.0 & 0.0 & 221.5 \\
\hline 16 & 74.0 & 0.0 & 360.2 & 74.0 & 0.0 & 85.1 & 1306.6 & 250.6 & 0.0 & 0.0 & 275.1 \\
\hline 17 & 94.2 & 0.0 & 346.1 & 94.2 & 0.0 & 346.1 & 1264.8 & 149.4 & 0.0 & 0.0 & 0.0 \\
\hline 18 & 63.2 & 0.0 & 237.4 & 63.2 & 0.0 & 221.9 & 7327.6 & 161.6 & 0.0 & 0.0 & 9.4 \\
\hline 19 & 85.7 & 0.0 & 265.0 & 85.7 & 0.0 & 220.4 & 1465.1 & 186.7 & 0.0 & 0.0 & 44.6 \\
\hline 20 & 65.8 & 0.0 & 0.0 & 65.8 & 0.0 & 0.0 & 1067.4 & 111.8 & 0.0 & 0.0 & 0.0 \\
\hline 21 & 46.2 & 0.0 & 455.8 & 46.2 & 0.0 & 371.6 & 1207.9 & 158.4 & 0.0 & 0.0 & 84.2 \\
\hline 22 & 58.8 & 0.0 & - 422.8 & 58.8 & 0.0 & 268.7 & 1507.0 & 256.3 & 0.0 & 0.0 & 154.7 \\
\hline 23 & 81.71 & 0.0 & 531.2 & 87.7 & 0.0 & 531.2 & 1261.8 & 128.7 & 0.0 & 0.0 & 0.0 \\
\hline 24 & 90.9 & 0.0 & 420.8 & 90.9 & 0.0 & 209.4 & 1722.2 & 263.2 & 0.0 & 0.0 & 271.4 \\
\hline 25 & 85.9 & 0.0 & $\therefore \quad 378.4$ & 85.9 & 0.0 & 748.7 & 1824.0 & 125.2 & 0.0 & 0.0 & 229.8 \\
\hline 26 & $62.2 !$ & 0.0 & $\quad 0.0$ & 62.2 & 0.0 & 0.0 & 1826.9 & 342.9 & 0.0 & 0.0 & 0.0 \\
\hline 27 & 69.21 & 0.0 & 0.0 & 69.2 & 0.0 & 0.0 & 1853.8 & 298.8 & 0.0 & 0.0 & 0.0 \\
\hline 28 & 90.11 & 0.0 & 300.1 & 90.1 & 0.0 & 0.0 & 1303.6 & 148.3 & 0.0 & 0.0 & 300.1 \\
\hline 29 & 64.11 & 0.6 & 326.8 & 64.1 & 0.0 & 218.2 & 1572.7 & 267.8 & 0.0 & 0.0 & 108.6 \\
\hline 30 & 86.6 & 0.0 & $: 293.2$ & 86.6 & 0.0 & 166.0 & 1214.0 & 105.5 & 0.0 & 0.0 & 127.2 \\
\hline 31 & 90.9 & 0.0 & 294.6 & 90.9 & 0.0 & 233.0 & 1211.0 & 171.8 & 0.0 & 0.0 & 61.3 \\
\hline Total & 2238.9 & 9.3 & $\vdots 11403.7$ & 2238.9 & $8.7^{\prime}$ & 7490.7 & 44204.2 & 6868.1 & 0.0 & 0.0 & 3913.1 \\
\hline Mean & 74.6 & 0.3 & 380.1 & 74.6 & 0.3 & 249.7 & 1474.1 & 228.9 & 0.0 & 0.0 & 130.4 \\
\hline
\end{tabular}

${ }^{*}$ On a 71.3 square meter solar collector surface 
Table 15. (August 1975) DAILY TOTAL HEAT RATES (MJ/DAY)

\begin{tabular}{|c|c|c|c|c|c|c|c|c|c|c|c|}
\hline \multirow{2}{*}{ Date } & \multicolumn{3}{|c|}{ Total Erisrg: Required } & \multicolumn{3}{|c|}{$\begin{array}{l}\text { Supplemental Energy } \\
\text { Required for }\end{array}$} & \multirow{2}{*}{$\begin{array}{l}\text { Incident * } \\
\text { Solar Energy }\end{array}$} & \multirow{2}{*}{$\begin{array}{l}\text { Solar Energy } \\
\text { Collected and } \\
\text { Stored }\end{array}$} & \multicolumn{3}{|c|}{$\begin{array}{l}\text { Solar Energy Delivered } \\
\text { from Storage to }\end{array}$} \\
\hline & $\begin{array}{l}\text { DHW } \\
\text { Load }\end{array}$ & $\begin{array}{c}\text { He } \vdots \vdots i r= \\
\text { Loje }\end{array}$ & $\begin{array}{c}\text { Cooling } \\
\text { Load }\end{array}$ & $\begin{array}{c}\text { DHW } \\
\text { Load } \\
\end{array}$ & $\begin{array}{l}\text { Heating } \\
\text { Load }\end{array}$ & $\begin{array}{l}\text { Cooling } \\
\text { Load }\end{array}$ & & & $\begin{array}{l}\text { DHW } \\
\text { Load }\end{array}$ & $\begin{array}{l}\text { Heating } \\
\text { Load }\end{array}$ & $\begin{array}{c}\text { Cooling } \\
\text { Load }\end{array}$ \\
\hline 1 & 78.3 & 0.0 & 1337.6 & 78.3 & 0.0 & 239.4 & 1632.5 & 230.0 & 0.0 & 0.0 & 92.2 \\
\hline 2 & 87.9 & 0.0 & $=0.0$ & 87.9 & 0.0 & 0.0 & 1803.0 & 7.2 & 0.0 & 0.0 & 0.0 \\
\hline 3 & 62.0 & 0.0 & 0.0 & 62.0 & 0.0 & 0.0 & 1931.5 & 336.5 & 0.0 & 0.0 & 0.0 \\
\hline 4 & 85.6 & 15.6 & 464.8 & 85.6 & 0.0 & 0.6 & 1880.7 & 330.4 & 0.0 & 15.6 & 464.1 \\
\hline 5 & 77.4 & 0.0 & 392.7 & 77.4 & 0.0 & 230.9 & 1590.7 & 282.9 & 0.0 & 0.0 & 161.7 \\
\hline 6 & 82.5 & 8.2 & 374.5 & 82.5 & 7.3 & 371.6 & 1043.5 & 96.7 & 0.0 & 0.9 & 62.9 \\
\hline 7 & 54.0 & 2.8 & 268.0 & 54.0 & 2.8 & 244.7 & 1160.1 & 155.8 & 0.0 & 0.0 & 23.3 \\
\hline 8 & 66.9 & 0.0 & 206.2 & 66.9 & 0.0 & 143.6 & 1877.7 & 320.1 & 0.0 & 0.0 & 62.6 \\
\hline 9 & 70.2 & 0.0 & 551.7 & 70.2 & 0.0 & 270.2 & 1767.1 & 271.6 & 0.0 & 0.0 & 281.5 \\
\hline 10 & 11.3 & 0.0 & 1266.9 & 11.3 & 0.0 & 266.9 & 6.0 & 0.0 & 0.0 & 0.0 & 0.0 \\
\hline 11 & 49.1 & 0.0 & 884.5 & 49.1 & 0.0 & 805.0 & 1076.4 & 153.9 & 0.0 & 0.0 & 79.4 \\
\hline 12 & 77.2 & 0.0 & 823.8 & 77.2 & 0.0 & 823.8 & 837.2 & 62.1 & 0.0 & 0.0 & 0.0 \\
\hline 13 & 90.3 & 0.0 & 12.6 & 90.3 & 0.0 & 12.6 & 293.0 & 0.0 & 0.0 & 0.0 & 0.0 \\
\hline 14 & 78.1 & 0.0 & 0.0 & 78.1 & 0.0 & 0.0 & 968.8 & 64.1 & 0.0 & 0.0 & 0.0 \\
\hline 15 & 85.9 & 0.0 & 0.0 & 85.9 & 0.0 & 0.0 & 1387.4 & 174.4 & 0.0 & 0.0 & 0.0 \\
\hline 16 & 79.8 & 0.0 & 0.0 & 79.8 & 0.0 & 0.0 & 1408.3 & 186.8 & 0.0 & 0.0 & 0.0 \\
\hline 17 & 68.8 & 0.0 & 0.0 & 68.8 & 0.0 & 0.0 & 1450.2 & 108.5 & 0.0 & 0.0 & 0.0 \\
\hline 18 & 74.9 & 0.0 & 789.7 & 74.9 & 0.0 & 574.4 & 1668.4 & 242.1 & 0.0 & 0.0 & 215.2 \\
\hline 19 & 69.2 & 0.0 & 806.3 & 69.2 & 0.0 & 762.3 & 1279.7 & 133.1 & 0.0 & 0.0 & 44.0 \\
\hline 20 & 72.3 & 0.0 & 997.9 & 72.3 & 0.0 & 996.7 & 980.7 & 51.8 & 0.0 & 0.0 & 1.2 \\
\hline 27 & 78.9 & 0.0 & 567.4 & 78.9 & 0.0 & 389.0 & 1393.3 & 263.7 & 0.0 & 0.0 & 178.4 \\
\hline 22 & 68.8 & 0.0 & 369.2 & 68.8 & 0.0 & 236.8 & 1432.2 & 189.3 & 0.0 & 0.0 & 132.4 \\
\hline 23 & 96.2 & 0.0 & 0.0 & 96.2 & 0.0 & 0.0 & 1453.1 & 270.5 & 0.0 & 0.0 & 0.0 \\
\hline 24 & 72.3 & 0.0 & 21.7 & 72.3 & 0.0 & 0.0 & 1656.5 & 194.3 & 0.0 & 0.0 & 21.7 \\
\hline 25 & 91.4 & 0.0 & 175.4 & 91.4 & 0.0 & 0.0 & 183.5 .9 & 266.4 & 0.0 & 0.0 & 175.4 \\
\hline 26 & 80.0 & 0.0 & $\therefore \quad 440.3$ & 80.0 & 0.0 & 76.5 & 1859.8 & 421.9 & 0.0 & 0.0 & 363.7 \\
\hline 27 & 100.0 & 0.0 & 808.5 & 100.0 & 0.0 & 649.1 & 1345.5 & 169.5 & 0.0 & 0.0 & 159.4 \\
\hline 28 & 108.3 & 0.0 & 986.7 & 108.3 & 0.0 & 778.9 & 1581.7 & 306.9 & 0.0 & 0.0 & 207.7 \\
\hline 29 & 93.4 & 0.0 & 971.9 & 93.4 & 0.1 & 644.3 & 1868.8 & 454.6 & 0.0 & 0.0 & 327.6 \\
\hline 30 & 90.9 & 0.0 & 842.3 & 90.0 & 0.0 & 494.8 & 1904.6 & 482.9 & 0.0 & 0.0 & 347.5 \\
\hline 31 & 87.7 & 0.0 & 982.7 & 87.7 & 0.0 & 618.0 & 1922.6 & 471.7 & 0.0 & 0.0 & 364.8 \\
\hline Total & 2389.5 & 26.7 & 13337.3 & 2389.5 & 10.2 & 9570.3 & 44302.8 & 6639.6 & 0.0 & 16.5 & 3767.1 \\
\hline Mean & 77.1 & 0.8 & 430.2 & 77.1 & 0.3 & 308.7 & 1429.2 & 214.2 & 0.0 & 0.5 & 121.5 \\
\hline
\end{tabular}

*On a 71.3 square meter solar collector surface 
Table 16. Monthly and Annual Values of Mean Daily Heat Rates

\begin{tabular}{|c|c|c|c|c|c|c|c|c|c|c|c|}
\hline \multirow{2}{*}{ Date } & \multicolumn{3}{|c|}{ Total Energy Required } & \multicolumn{3}{|c|}{\begin{tabular}{|c|} 
Supplemental \\
Energy Required \\
\end{tabular}} & \multirow{2}{*}{$\begin{array}{l}\text { Incident } \\
\text { Solar Energy } \\
\star\end{array}$} & \multirow{2}{*}{$\begin{array}{l}\text { Solar. Energy } \\
\text { Collected }\end{array}$} & \multicolumn{3}{|c|}{$\begin{array}{l}\text { Solar Energy } \\
\text { Delivered to }\end{array}$} \\
\hline & $\begin{array}{l}\text { OHW } \\
\text { Load }\end{array}$ & $\begin{array}{l}\text { Heating } \\
\text { Load }\end{array}$ & $\begin{array}{c}\text { Cooling } \\
\text { Load }\end{array}$ & $\begin{array}{c}\text { To } \\
\text { DHW }\end{array}$ & $\begin{array}{c}\text { To } \\
\text { Heating }\end{array}$ & $\begin{array}{c}\text { To } \\
\text { Cooling }\end{array}$ & & & DHW & Heating & Cooling \\
\hline September 1974 & 38.9 & 10.5 & 354.1 & 9.6 & 0.9 & 239.2 & 1106.3 & 249.1 & 29.3 & 9.6 & 114.8 \\
\hline October 1974 & 52.6 & 10.6 & 210.4 & 10.9 & 0.3 & 150.9 & 849.2 & 179.1 & 41.7 & 10.3 & 59.4 \\
\hline November 1974 & 40.8 & 116.5 & 9.5 & 11.8 & 0.3 & 9.5 & 780.4 & 152.9 & 29.0 & 116.2 & - \\
\hline December 1974 & 41.2 & 225.6 & 0.5 & 14.1 & 56.3 & 0.5 & 956.8 & 227.5 & 27.1 & 169.1 & - \\
\hline January 1975 & 47.0 & 252.6 & - & 25.9 & 79.1 & - & 998.7 & 262.2 & 21.1 & 173.4 & - \\
\hline February 1975 & 88.5 & 236.9 & - & 40.8 & 48.6 & - & 1273.7 & 313.4 & 47.7 & 188.3 & - \\
\hline March 1975 & 104.5 & 129.6 & - & 58.6 & 46.9 & - & 1112.3 & 134.3 & 45.9 & 82.7 & - \\
\hline April 1975 & 156.4 & 121.5 & - & 98.1 & 30.8 & - & 1512.9 & 230.0 & 58.3 & 90.7 & - \\
\hline May 1975 & 161.1 & 15.3 & - & 93.4 & 0.7 & - & 1216.9 & 182.7 & 67.7 & 14.6 & - \\
\hline June 1975 & 131.8 & 1.6 & 310.8 & 78.7 & 0.0 & 272.7 & 1375.4 & 198.7 & 53.1 & 1.6 & 38.1 \\
\hline July 1975 & 74.6 & 0.3 & 380.1 & 74.6 & 0.3 & 249.7 & 1474.1 & 228.9 & 0.0 & 0.0 & 130.4 \\
\hline August 1975 & 77.1 & 0.8 & 430.2 & 77.1 & 0.3 & 308.7 & 1429.2 & 214.2 & 0.0 & 0.5 & 121.5 \\
\hline Annual & 84.5 & 93.5 & 141.3 & 49.5 & 22.0 & 102.6 & 1173.8 & 214.4 & 35.7 & 71.4 & 38.7 \\
\hline
\end{tabular}

*On a 71.3 square meter solar collector surface 
the heating demand. Whenever the temperature of the building fell below a preset value at the thermostat $\left(\mathrm{e} . \mathrm{g} ., 20^{\circ} \mathrm{C}, 68^{\circ} \mathrm{F}\right)$, the solar portion of the system delivered heat to the heating coils. If the building temperature reached $19^{\circ} \mathrm{C}\left(66^{\circ} \mathrm{F}\right)$, or the thermal storage unit temperature was below some preset selected value $\left(\mathrm{e} . \mathrm{g} ., 38^{\circ} \mathrm{C}, 100^{\circ} \mathrm{F}\right)$, the auxiliary began supplying heat to the building.

For cooling the decision to use the auxiliary gas boiler was based only on a selected preset temperature of the thermal storage unit. Above this particular temperature $\left(\mathrm{e} . \mathrm{g} ., 82^{\circ} \mathrm{C}, 130^{\circ} \mathrm{F}\right)$, the solar portion of the system delivers heat to the cooling unit and is used to meet the cooling load; below this temperature the auxiliary delivers the heat to the cooling unit.

\section{QUALITY OF THERMAL PERFORMANCE OF THE SYSTEM}

The quality of thermal performance of the system is determined by the departure of measured room temperature from the design temperature over the reporting period. In CSU Solar House I, the design temperature is selected by setting the room thermostat. For winter heating operations and summer cooling operations the settings were $20^{\circ} \mathrm{C}\left(68^{\circ} \mathrm{F}\right)$. and $22^{\circ} \mathrm{C}\left(72^{\circ} \mathrm{F}\right)$, respectively. Table 17 shows the daily mean building temperatures for the reporting period (1 September 1974 to 31 August 1975). Note that the cooling system was not operational in the spring until I June.

\section{SOLAR CONTRIBUTION TO ENERGY REQUIREMENTS}

Tables 18 through 20 show the daily and monthly values of per cent. solar contribution to energy requirements for domestic hot water, (DHW), space heating, and space cooling, respectively. The annual solar percentages are 42 per cent for DHW, 76 per cent for space heating; and 27 per 
Table 17. DAILY MEAN BUilding TEMPERATURE $\left({ }^{\circ} \mathrm{C}\right)$

\begin{tabular}{|c|c|c|c|c|c|c|c|c|c|c|c|c|}
\hline Date & $\begin{array}{l}\text { Sept } \\
1974\end{array}$ & $\begin{array}{l}0 c t \\
1974\end{array}$ & $\begin{array}{c}\text { Nov } \\
1974\end{array}$ & $\begin{array}{c}\text { Dec } \\
1974 \\
\end{array}$ & $\begin{array}{c}\text { Jan } \\
1975 \\
\end{array}$ & $\begin{array}{c}\text { Feb } \\
1975 \\
\end{array}$ & $\begin{array}{c}\text { March } \\
1975\end{array}$ & $\begin{array}{c}\text { April } \\
1975\end{array}$ & $\begin{array}{c}\text { May } \\
1975 \\
\end{array}$ & $\begin{array}{l}\text { June } \\
1975 \\
\end{array}$ & $\begin{array}{l}\text { July } \\
1975 \\
\end{array}$ & $\begin{array}{c}\text { August } \\
1975\end{array}$ \\
\hline 1 & 21.7 & 22.8 & 18.6 & 18.2 & 18.4 & 19.4 & 19.8 & 18.9 & 20.5 & 20.6 & 25.5 & 24.8 \\
\hline 2 & 19.4 & 23.4 & 17.9 & 19.7 & 17.8 & 19.7 & 19.5 & 18.8 & 27.8 & 23.2 & 23.2 & 25.1 \\
\hline 3 & 20.3 & 23.4 & 17.7 & 19.3 & 18.6 & 20.0 & 20.2 & 19.5 & 21.3 & 25.9 & 23.9 & 26.9 \\
\hline 4 & 21.8 & 23.0 & 18.1 & 20.9 & 18.4 & $7^{0} .1$ & 21.1 & 20.6 & $? ? .3$ & 25.4 & 22.0 & 26.9 \\
\hline 5 & 22.4 & 22.3 & 18.6 & 19.8 & 18.2 & 18.6 & 21.3 & 21.1 & 22.7 & 25.6 & 22.7 & 25.9 \\
\hline 6 & 22.3 & 27.6 & 19.9 & 20.0 & 19.2 & 19.2 & 19.8 & 21.0 & 20.3 & 25.0 & - & 25.9 \\
\hline 7 & 22.3 & 22.3 & 120.5 & 18.4 & 18.9 & 19.4 & 19.0 & 20.9 & 21.3 & 22.2 & 23.2 & 27.1 \\
\hline 8 & 22.7 & 22.4 & 120.4 & 18.4 & 18.8 & 17.9 & 19.2 & 20.5 & 22.0 & 22.1 & 22.8 & 27.4 \\
\hline 9 & 22.5 & 22.8 & 18.4 & 19.7 & 18.5 & 77.9 & 19.3 & 20.3 & $21.7^{\circ}$ & 20.4 & 25.6 & 26.3 \\
\hline 10 & 22.1 & 23.0 & 18.3 & 19.0 & 17.9 & 19.7 & 18.2 & 19.9 & 22.2 & 20.4 & 23.9 & 21.1 \\
\hline 11 & 21.4 & 22.9 & 18.2 & - & 17.5 & 20.1 & 19.6 & 19.1 & 21.7 & 21.7 & 24.4 & 21.8 \\
\hline 12 & 18.6 & 21.9 & - & $=$ & 17.7 & 21.7 & 19.2 & 18.5 & 21.5 & 22.1 & 23.2 & 22.3 \\
\hline 13 & 19.6 & 21.9 & $=$ & $=$ & 19.4 & 22.2 & 19.6 & 18.8 & 21.8 & 23.3 & 23.6 & 23.7 \\
\hline 14 & 20.7 & 22.6 & - & $=$ & 20.2 & 20.0 & 20.3 & 20.7 & 23.4 & 22.5 & 24.8 & 21.7 \\
\hline 15 & 20.8 & - & $=$ & - & 19.7 & 18.3 & 20.0 & 21.7 & 24.8 & 22.9 & 25.2 & 23.1 \\
\hline 16 & 20.9 & 24.7 & $=$ & $=$ & 19.2 & 18.2 & 19.7 & 23.0 & 25.0 & 22.7 & 25.0 & 23.2 \\
\hline 17 & 27.3 & 23.0 & - & $=$ & 20.0 & 19.8 & 20.9 & 21.2 & 25.0 & 22.3 & 24.9 & 24.5 \\
\hline 18 & 20.7 & - & - & - & 19.3 & 20.7 & 27.7 & 19.7 & 24.2 & 22.4 & 27.2 & 23.7 \\
\hline 19 & - & - & 19.2 & 20.0 & 18.4 & 20.5 & 22.5 & 19.1 & 24.8 & 23.5 & 28.1 & 23.3 \\
\hline 20 & 20.4 & - & 19.0 & 19.4 & 18.8 & 20.5 & 23.7 & 19.6 & 23.2 & 23.0 & 26.9 & 23.0 \\
\hline 27 & 20.2 & 24.6 & 21.9 & 19.5 & 18.5 & 19.0 & 21.5 & 20.9 & 19.8 & 22.4 & 25.3 & 23.8 \\
\hline 22 & 20.3 & 24.0 & 119.8 & 19.2 & 18.5 & 19.4 & 19.6 & 27.3 & 21.1 & 23.0 & 26.0 & 25.1 \\
\hline 23 & 21.9 & 21.4 & 19.9 & 18.2 & 19.2 & 19.4 & 18.5 & 23.0 & 20.5 & 23.3 & 25.2 & 27.7 \\
\hline 24 & 22.0 & 20.5 & $\therefore \quad 19.7$ & 19.2 & 20.3 & 21.2 & 18.7 & 27.1 & 20.9 & 23.6 & 24.2 & 27.3 \\
\hline 25 & 22.1 & 20.9 & 20.0 & 19.2 & - & 19.9 & 79.5 & 23.3 & 21.3 & 23.1 & 26.0 & 24.2 \\
\hline 26 & 22.5 & 19.9 & 18.8 & 18.7 & - & 19.7 & 18.5 & 24.4 & 21.1 & - & 29.0 & 24.7 \\
\hline 27 & 21.2 & 20.3 & 18.2 & 19.7 & 18.7 & 20.5 & 17.6 & 21.1 & 22.3 & - & 29.8 & 24.4 \\
\hline 28 & 20.3 & 20.6 & 117.6 & 19.7 & 19.4 & 20.0 & 18.2 & 19.9 & 20.6 & $=$ & 27.4 & 23.0 \\
\hline 29 & 21.6 & 19.6 & 17.2 & 19.3 & 19.0 & & - & 20.0 & 19.2 & - & 27.1 & 22.7 \\
\hline+30 & 21.9 & 19.3 & 17.5 & 19.2 & 19.4 & & 19.4 & 19.7 & 20.5 & - & 27.6 & 22.5 \\
\hline 31 & & 19.1 & & 18.7 & 19.5 & & 19.0 & & 20.7 & & 25.8 & 22.7 \\
\hline $\begin{array}{l}\text { Month } \\
\text { Avera }\end{array}$ & 21.2 & 22.0 & 18.9 & 19.3 & 18.9 & 19.7 & 19.8 & 20.6 & 21.9 & 22.9 & 25.3 & 24.4 \\
\hline
\end{tabular}


Table 18. Solar Contribution to Energy Requirements Domestic Hot Water (DHW) Per Cent of Load

\begin{tabular}{|c|c|c|c|c|c|c|c|c|c|c|c|c|}
\hline Date & $\begin{array}{l}\text { Sept } \\
1974\end{array}$ & $\begin{array}{l}\text { Oct } \\
1975\end{array}$ & $\begin{array}{c}\text { Nov } \\
1974 \\
\end{array}$ & $\begin{array}{c}\text { Dec } \\
1974 \\
\end{array}$ & $\begin{array}{r}\text { Jan } \\
1975 \\
\end{array}$ & $\begin{array}{l}\text { Feb } \\
1975\end{array}$ & $\begin{array}{c}\text { March } \\
1975 \\
\end{array}$ & $\begin{array}{c}\text { April } \\
1975 \\
\end{array}$ & $\begin{array}{l}\text { May } \\
1975 \\
\end{array}$ & $\begin{array}{l}\text { June } \\
1975 \\
\end{array}$ & $\begin{array}{l}\text { July } \\
1975\end{array}$ & $\begin{array}{c}\text { August } \\
1975\end{array}$ \\
\hline 1 & 74.5 & 58.0 & 78.0 & 73.8 & 63.0 & 78.7 & 81.5 & 9.5 & 42.2 & 38.7 & 0.0 & 0.0 \\
\hline 2 & 28.6 & 48.2 & $: 32.0$ & 82.0 & 0.0 & 84.8 & 73.8 & 21.7 & 35.5 & 48.6 & 0.0 & 0.0 \\
\hline 3 & 80.3 & 60.5 & 26.0 & 87.5 & 68.6 & 57.9 & 73.5 & 43.9 & 54.7 & 56.7 & 0.0 & 0.0 \\
\hline 4 & 81.4 & 76.8 & 84.5 & 76.4 & 76.7 & 45.2 & 80.1 & 26.3 & 53.1 & 50.0 & 0.0 & 0.0 \\
\hline 5 & 77.9 & 73.0 & 82.3 & 41.8 & 63.1 & 8.9 & 79.2 & 59.8 & 64.4 & 48.9 & 0.0 & 0.0 \\
\hline 6 & 85.8 & 81.6 & $\therefore 100.0$ & 86.5 & 73.9 & 50.9 & 79.4 & 45.4 & 47.3 & 43.4 & 0.0 & 0.0 \\
\hline 7 & 0.0 & 93.6 & 100.0 & 27.5 & 77.5 & 70.7 & 38.8 & 35.7 & 37.8 & 41.4 & 0.0 & 0.0 \\
\hline 8 & 81.6 & 100.0 & $\div \quad 79.8$ & 81.7 & 33.5 & 0.0 & 47.8 & 32.0 & 17.0 & 40.4 & 0.0 & 0.0 \\
\hline 9 & 70.4 & 80.2 & 668.3 & 72.4 & 32.3 & 18.2 & 0.0 & 29.0 & 55.5 & 30.3 & 0.0 & 0.0 \\
\hline 10 & 84.1 & 77.8 & 173.5 & 100.0 & 33.5 & 2.0 & 0.0 & 3.1 & 35.0 & 23.5 & 0.0 & 0.0 \\
\hline 11 & 71.9 & 72.3 & 75.8 & 0.0 & 12.0 & 59.0 & 0.0 & 16.9 & 25.8 & 35.5 & 0.0 & 0.0 \\
\hline 12 & 17.2 & 70.1 & $\therefore \quad 0,0$ & 0.0 & 43.1 & 69.1 & 59.8 & 41.0 & 27.7 & 15.6 & 0.0 & 0.0 \\
\hline 13 & 73.4 & 69.3 & 0.0 & 0.0 & 15.4 & 62.7 & 62.1 & 36.1 & 39.9 & 27.2 & 0.0 & 0.0 \\
\hline 14 & 71.5 & 51.6 & $: \quad 0.0$ & 0.0 & 72.7 & 32.7 & 84.7 & 43.2 & 31.5 & 15.1 & 0.0 & 0.0 \\
\hline 15 & 65.8 & 0.0 & 0.0 & 0.0 & 25.3 & 20.0 & 55.7 & 43.0 & 18.1 & 26.9 & 0.0 & 0.0 \\
\hline 16 & 82.2 & 78.8 & 0.0 & 0.0 & 11.7 & 36.1 & 0.0 & 57.0 & 33.8 & 14.6 & 0.0 & 0.0 \\
\hline 17 & 67.0 & 100.0 & 0.0 & 0.0 & 55.2 & 23.6 & 49.3 & 37.4 & 24.7 & 52.5 & 0.0 & 0.0 \\
\hline 18 & 100.0 & 0.0 & 0.0 & 0.0 & 46.4 & 57.3 & 45.6 & 40.0 & 20.3 & 26.4 & 0.0 & 0.0 \\
\hline 19 & 0.0 & 0,0 & 47.0 & 58.5 & 54.8 & 56.1 & 45.7 & 38.5 & 43.8 & 53.6 & 0.0 & 0.0 \\
\hline 20 & 62.1 & 0.0 & 7.6 & 24.1 & 59.7 & 61.4 & 57.3 & 30.5 & 44.5 & 43.1 & 0.0 & 0.0 \\
\hline 21 & 88.4 & 100.0 & .57 .3 & 46.8 & 52.2 & 55.1 & 44.3 & 70.2 & 42.4. & 45.7 & 0.0 & 0.0 \\
\hline 22 & 65.1 & 1100.0 & 75.3 & 66.5 & 24.0 & 71.9 & 37.3 & 46.4 & 47.9 & 44.1 & 0.0 & 0.0 \\
\hline .23 & 81.1 & 75.0 & 86.7 & 7.9 & 38.9 & 34.6 & 0.0 & 46.5 & 57.4 & 17.1 & 0.0 & 0.0 \\
\hline 24 & 87.9 & 100.0 & 69.7 & 69.7 & 5.2 & 92.3 & 40.8 & 54.3 & 49.2 & 58.4 & 0.0 & 0.0 \\
\hline 25 & 72.3 & 993.5 & $: 65.3$ & 67.2 & 0.0 & 80.7 & 43.8 & 33.7 & 52.4 & 53.2 & 0.0 & 0.0 \\
\hline 26 & 93.7 & 186.5 & $i \quad 77.7$ & 12.7 & 0.0 & 70.7 & 9.7 & 27.0 & 53.3 & 0.0 & 0.0 & 0.0 \\
\hline 27 & 55.9 & $! 79.7$ & $=36.1$ & 83.7 & 0.0 & 85.8 & 12.2 & 13.1 & 47.7 & 0.0 & 0.0 & 0.0 \\
\hline 28 & 88.3 & 92.0 & 41.8 & 62.0 & 37.2 & 77.8 & 24.1 & 51.3 & 39.9 & 0.0 & 0.0 & 0.0 \\
\hline 29 & 71.5 & 185.7 & 38.6 & 40.6 & 55.7 & & 0.0 & 28.4 & 19.5 & 0.0 & 0.0 & 0.0 \\
\hline 30 & 72.5 & 75.2 & 82.1 & 72.8 & 0.0 & & 43.8 & 22.1 & 35.7 & 0.0 & 0.0 & 0.0 \\
\hline 31 & & 27.8 & & 56.7 & 53.3 & & 12.8 & & 29.8 & & 0.0 & 0.0 \\
\hline $\begin{array}{l}\text { Month? } \\
\text { Averag }\end{array}$ & 75.3 & 79.3 & $\quad 71.0$ & 65.8 & 44.9 & 53.9 & 43.9 & 37.3 & 42.0 & 40.3 & 0.0 & 0.0 \\
\hline
\end{tabular}


Table 19. Solar Contribution to Energy Requirements

Space Heating (Per Cent of Load)

\begin{tabular}{|c|c|c|c|c|c|c|c|c|c|c|c|c|}
\hline Date & $\begin{array}{l}\text { Sept } \\
1974\end{array}$ & $\begin{array}{l}0 c t \\
197 \div\end{array}$ & $\begin{array}{c}\text { Nov } \\
1974 \\
\end{array}$ & $\begin{array}{c}\text { Dec } \\
1974\end{array}$ & $\begin{array}{c}\text { Jan } \\
1975\end{array}$ & $\begin{array}{c}\text { Feb } \\
1975\end{array}$ & $\begin{array}{c}\text { March } \\
1975\end{array}$ & $\begin{array}{l}\text { April } \\
1975\end{array}$ & $\begin{array}{c}\text { May } \\
1975 \\
\end{array}$ & $\begin{array}{l}\text { June } \\
1975 \\
\end{array}$ & $\begin{array}{l}\text { July } \\
1975\end{array}$ & $\begin{array}{c}\text { August } \\
1975\end{array}$ \\
\hline 1 & - & - & $: 92.1$ & 100.0 & 100.0 & 32.7 & 96.8 & 7.5 & 100.0 & 100.0 & - & - \\
\hline 2 & - & $=$ & 100.0 & 100.0 & 84.8 & $100: 0$ & 92.2 & 45.5 & 100.0 & - & - & $=$ \\
\hline 3 & 89.5 & - & 100.0 & 100.0 & 41.2 & 100.0 & 93.6 & 96.6 & 100.0 & - & 0.0 & - \\
\hline 4 & - & $=$ & $100: 0$ & 100.0 & 100.0 & 1000 & م. & 100.0 & - & $=$ & 0.0 & 100.0 \\
\hline 5 & - & - & 100.0 & 96.3 & 100.0 & 52.0 & 100.0 & 100.0 & - & - & 0.0 & \\
\hline 6 & 100.0 & $=$ & $: 100.0$ & 100.0 & 100.0 & 4.6 & 100.0 & 100.0 & 71.8 & - & - & 10.7 \\
\hline 7 & - & - & $! \quad-$ & 100.0 & 99.6 & 33.4 & 100.0 & 100.0 & 1000 & 0.0 & $=$ & 0.0 \\
\hline 8 & $\overline{-}$ & $\overline{-}$ & 1100.0 & 100.0 & 100.0 & 100.0 & 1000 & 100.0 & 100.0 & 0.0 & - & - \\
\hline 9 & - & - & 100.0 & 100.0 & 100.0 & 24.2 & 100.0 & 100.0 & 100.0 & - & - & - \\
\hline 10 & - & - & 100.0 & 100.0 & 59.1 & 33.0 & 100.0 & 100.0 & - & 100.0 & - & $=$ \\
\hline 11 & - & - & 100.0 & - & 10.9 & 100.0 & 0.0 & 10.2 & - & 100.0 & - & $=$ \\
\hline 12 & 100.0 & $=$ & $1 \quad$ & $=$ & 40.8 & 86.9 & 39.4 & 40.7 & 100.0 & 78.7 & - & - \\
\hline 13 & 80.1 & - & $1-$ & $=$ & 30.8 & 100.0 & 100.0 & 100.0 & 100.0 & $\therefore$ & - & - \\
\hline 14 & 100.0 & $=$ & $1=$ & $=$ & 54.0 & 100.0 & 100.0 & 100.0 & 100.0 & 0.0 & - & - \\
\hline 15 & - & - & $=$ & - & 100.0 & 99.5 & 100.0 & 100.0 & - & - & $=$ & - \\
\hline 16 & - & - & - & - & 91.1 & 87.8 & - & - & - & 100.0 & - & - \\
\hline 77 & - & - & - & $=$ & 57.2 & 100.0 & 100.0 & 100.0 & - & - & - & - \\
\hline 18 & $=$ & $=$ & $1-$ & - & 100.0 & 100.0 & 100.0 & 100.0 & $=$ & - & - & - \\
\hline 19 & - & - & $: \quad 100.0$ & 98.7 & 100.0 & 100.0 & 100.0 & 100.0 & - & - & $=$ & - \\
\hline 20 & - & - & 100.0 & 71.3 & 100.0 & 98.4 & - & 100.0 & - & $=$ & - & - \\
\hline 27 & 100.0 & - & $i \quad-$ & 11.9 & 100.0 & 100.0 & 100.0 & 100.0 & 100.0 & - & - & $=$ \\
\hline 22 & - & - & $\therefore \quad 100.0$ & 64.2 & 100.0 & 100.0 & 100.0 & 100.0 & 100.0 & $=$ & - & $=$ \\
\hline 23 & $=$ & - & $\therefore 100.0$ & 44.3 & 97.7 & 100.0 & 100.0 & $=$ & 100.0 & - & - & $=$ \\
\hline 24 & $=$ & $=$ & $\therefore \quad 100.0$ & 44.5 & 100.0 & 100.0 & 93.6 & $=$ & 100.0 & - & - & - \\
\hline 25 & - & - & $i \quad 96.9$ & 55.5 & - & 100.0 & 100.0 & - & - & - & - & $\therefore$ \\
\hline 26 & - & $=$ & $\therefore \quad 100.0$ & 82.7 & $=$ & 100.0 & 62.8 & - & 100.0 & $=$ & $=$ & - \\
\hline 27 & - & - & $\therefore \quad 99.4$ & 43.3 & 28.7 & 98.1 & 0.1 & 100.0 & - & - & - & - \\
\hline 28 & 100.0 & - & $\therefore \quad 100.0$ & 100.0 & 18.9 & 100.0 & 37.7 & 100.0 & $=$ & - & - & $=$ \\
\hline 29 & - & 100.0 & $\therefore \quad 100.0$ & 100.0 & 100.0 & & - & 100.0 & 100.0 & - & 100.0 & 0.0 \\
\hline 30 & $=$ & 94.5 & 100.0 & 100.0 & 100.0 & & 100.0 & 100.0 & 100.0 & & - & - \\
\hline 31 & & 100.0 & $:$ & 100.0 & 55.9 & & 100.0 & & - & & - & - \\
\hline $\begin{array}{l}\text { Montin } \\
\text { Averag }\end{array}$ & 91.6 & 97.5 & 99.7 & 75.0 & 68.7 & 79.5 & 86.3 & 74.7 & 95.2 & 97.4 & 6.4 & 61.7 \\
\hline
\end{tabular}


Table 20. Solar Contribution to Energy Requirements Space Cooling (Per Cent of Load)

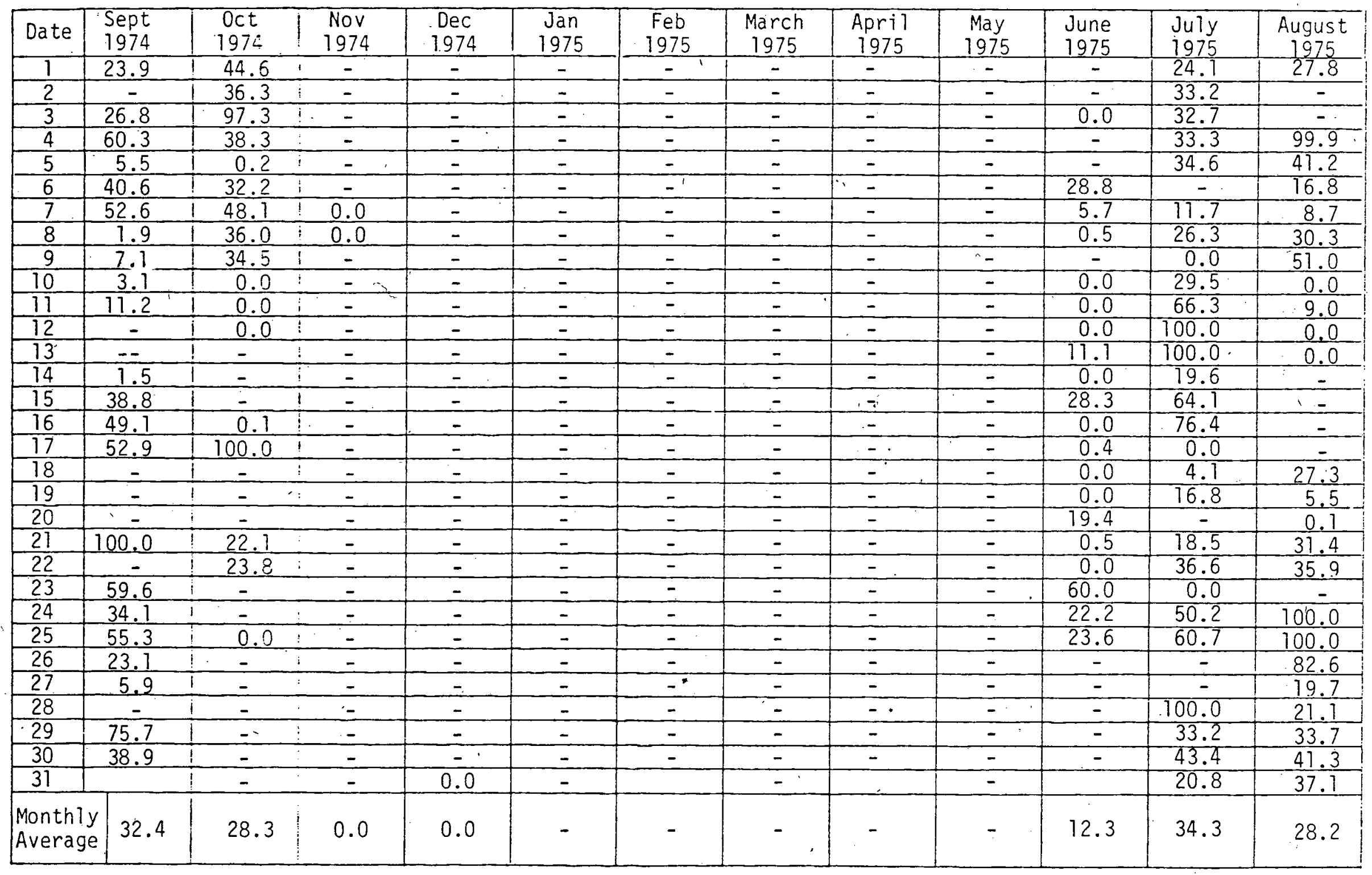


cent for space cooling. These values, however, were heavily influenced by the experimental nature of the project and do not adequateiy show the normal operation of the system. By accounting for these extraordinary effects (see sections on Heating System Performance and Cooling System Performance), the annual percentage carried by solar becomes 49 per cent of the domestic hot water load, 87 per cent of the space heating load, and 81 per cent of the space cooling load.

\section{MONTHLY AND ANNUAL ENERǴY OR FUEL SAVINGS}

Table 21 shows the monthly and annual quantities of gas heat saved by use of the solar' heating and cooling system. Savings of energy for domestic water heating are tabulated in column [1]. These values are obtained from Tables 4 through 15 and account for the heat losses from the domestic hot water solar preheat tank and the combustion efficiency of the auxiliary domestic hot water unit.

Energy savings for space heating (column [2]) correspond to the useful solar energy delivered to thermal storage for all heating purposes, less that used for domestic hot water heating (heat losses from storage and piping being useful for space heating). Heat losses from the domestic hot water preheat tank also contribute to meeting the heating load and are included in the figures in column [2].

In sunmer operation, the energy savings in column [3] are solar delivery to the cooling load less the energy required for producing sufficient cooling to match (remove) the heat losses from the solar system (storage tank, DHW preheat tank, piping, valves, etc.). Column [4] shows the effects on the energy savings for cooling operations if it is assumed that the solar system heat losses did not contribute to the cooling load (i.e., if the solar equipment were located outside the building's air 
Table 21. Monthly and Annual Energy Savings (MJ)

\begin{tabular}{|c|c|c|c|c|c|c|}
\hline & [1] & [2] & [3] & [4] & {$[5]$} & [6] \\
\hline Month & $\begin{array}{l}\text { Domestic Hot } \\
\text { Water (DHW)I }\end{array}$ & $\begin{array}{c}\text { Space } \\
\text { Heating } 2 \\
\end{array}$ & \multicolumn{2}{|c|}{ Space Cooling 2} & \multicolumn{2}{|c|}{ Totals } \\
\hline September, 1974 & 627.1 & 790.9 & 986.3 & 4041.5 & 2404.3 & 5459.5 \\
\hline October, 1974 & 1118.2 & 3544.7 & .1449 .3 & 2198.0 & 6112.2 & 6860.9 \\
\hline November, 1974 & 504.4 & 5100.9 & 0.0 & 0.0 & 5605.3 & 5605.3 \\
\hline December, 1974 & 447.5 & 8146.1 & 0.0 & 0.0 & 8593.6 & 85.93 .6 \\
\hline January, 1975 & 336.5 & 9554.6 & 0.0 & 0.0 & 9891.1 & 9891.1 \\
\hline February, 1975 & 1269.1 & 9478.2 & 0.0 & 0.0 & 10747.3 & 10747.3 \\
\hline March, 1975 & 1708.6 & 4739.0 & 0.0 & 0.0 & 6447.6 & 6447.6 \\
\hline April, 1975 & 1812.6 & 6722.7 & 0.0 & 0.0 & 8535.3 & 8535.3 \\
\hline May, 1975 & 2273.2 & 4777.8 & 0.0 & 0.0 & 7051.0 & 7051.0 \\
\hline June, 1975 & 1508.2 & 598.4 & -1273.4 & 1156.1 & 703.2 & 3262.7 \\
\hline July, 1975 & $0.0^{*}$ & 0.0 & 1363.3 & 4749.0 & 1363.3 & 4749.0 \\
\hline August, 1975 & $0.0^{*}$ & 0.0 & 1251.8 & 4571.7 & 1251.8 & 4571.7 \\
\hline $\begin{array}{l}\text { Annual } \\
\text { Totals }\end{array}$ & 11605.4 & 53453.3 & 3647.3 & 16716.3 & 68706.0 & 81755.0 \\
\hline
\end{tabular}

1. - Gas water heater has a measured combustion efficiency of $76.0 \%$

2 - Gas boiler has a measured combustion efficiency of $82.4 \%$

* - DH'd solar preheat tank by-passed during months of July and August 
conditioned space). Sample calculations to obtain the values in Table 21 are shown below.

\section{Sample Calculations - Table 21}

Column [1]-For September the heat losses from the DHW solar preheat tank $\left(Q_{L}\right.$ (preheat)) were calculated to be $373 \mathrm{MJ} /$ month (based on the average solar preheat tank temperatures and the experimentally determined heat loss rate for a particular temperature difference). The useful solar energy delivered to the $\mathrm{DHW}$ load $\left(Q_{u}(D H W)\right)$ is given by:

$$
Q_{u}(D H W)=Q_{D H W}-Q_{L}(\text { preheat })
$$

where $Q_{D H W}$ is the solar heat delivered to DHW load (1 isted in Table 4). The energy savings, $E_{D H W}$, 1 isted in column [1] of Table 21 is then $Q_{u}$ (DHW) divided by the auxiliary DHW unit's combustion efficiency $(76 \%)$ Thus:

$$
E_{D H W}(\text { column }[1])=\frac{Q_{D H W}-Q_{L} \text { (preheat) }}{0.76}
$$

For September:

$$
E_{\text {DHW }}(\text { column }[1])=\frac{848.7 \mathrm{MJ}-373.0 \mathrm{MJ}}{0.76}=627.1 \mathrm{MJ}
$$

Column [2] - The energy savings for space heating, $E_{H}$, is the total useful energy delivered to the storage unit, $Q_{u}$, less that heat del ivered to the DHW system, $Q_{u}$ (DHW), divided by the gas boiler combustion efficiency, "B. This is due to the fact that all heat: losses from storage, piping, and the DHW system contribute to the solar space heating contribution.

For February (from Table 9):

$$
Q_{u}=8774.5 \mathrm{MJ} \quad E_{D H W}=1269.1 \mathrm{MJ}
$$


Now:

$$
Q_{u}(D H W)=0.76 E_{D H W}=964.5 \mathrm{MJ}
$$

and

$$
\begin{aligned}
& E_{H}=\frac{Q_{u}-Q_{u}(D H W)}{n_{B}}=\frac{8774.5 \mathrm{MJ}-964.5 \mathrm{MJ}}{0.824} \\
& E_{H}=9478.2 \mathrm{MJ}
\end{aligned}
$$

Column [3] - Q AIRS is the amount of solar heat delivered to the cooling unit. However, the heat losses from the solar system add to the cooling load, and therefore must be accounted for in determining the energy savings for space cooling, $E_{C}$. Therefore:

$$
\eta_{B} E_{C}=Q_{A I R S}-\left\{Q_{U}-Q_{D H W}-Q_{H C S}-Q_{A I R S}-\Delta_{S}\right\}
$$

where

$Q_{u}$ is the useful energy delivered to storage,

$\mathrm{Q}_{\mathrm{DHW}}$ is the storage heat delivered to domestic hot water,

$Q_{H C S}$ is the storage heat delivered to space heating,

$Q_{A I R S}$ is the storage heat delivered to space cooling,

and $\Delta Q_{S}$ is, the change in storage heat.

For June (from Tables 13 and 25):

$$
\begin{aligned}
& E_{C} n_{B}=952.6 \mathrm{MJ}-\{4968.6-1327.2-38.9-952.6-(21.6 / \text { day })(30 \text { days })\} M J \\
& E_{C} n_{B}=-1049.3 \mathrm{MJ} \\
& E_{C}=-1273.4 \mathrm{MJ}
\end{aligned}
$$

Column [4] - Assuming no solar heat losses contributing to the cooling load, we can obtain the energy savings for space cooling by:

$$
E_{C}(\text { column }[4])=Q_{A I R S} / n_{B}
$$


For June (from Table 13),

$$
E_{c}(\operatorname{column}[4])=952.6 \mathrm{MJ} / .824=1156.1 \mathrm{MJ}
$$

Column [5]

$$
\text { Column [5] = column [1] + column [2]+ column [3] }
$$

Column [6]

Colume $[6]=$ column $[1]+\operatorname{column}[2]+\operatorname{column}[4]$.

\section{ENERGY AND MASS BALANCES}

Energy balances of significant energy flow rates in and out of a specific component can be obtained from the data presented. Table 22 shows energy supply, withdrawal, and accumulation during several days. The distinctive difference between winter and summer heat losses is due to the difference in storage temperatures. The average storage temperature during the days shown in Table 22 were $50.7^{\circ} \mathrm{C}$ (winter) and $78.6^{\circ} \mathrm{C}$ (summer). At these temperatures heat is 10st at rates of $47.2 \mathrm{MJ} /$ day (winter) and $85.9 \mathrm{MJ} /$ day (summer), at house temperatures of $20^{\circ} \mathrm{C}$ and $22^{\circ} \mathrm{C}$, respectively.

Table 23 identifies the critical parameters which effect the thermal storage heat losses, including the thermal storage temperature, the thermal storage capacity at the end of each day, the daily change in thermal storage capacity, the thermal storage heat balance, the total solar heat to loads, and the heating system daily heat loss. In evaluating Table 23, it is important to know the source of the data presented. For example: 
Table 22. Selected Energỳ Balances on Thermal Storage Unit

\begin{tabular}{|c|c|c|c|c|c|c|c|c|}
\hline \multirow{2}{*}{ Date } & \multirow{2}{*}{$\begin{array}{l}\text { Del ivered } \\
\text { to Storage } \\
\text { (MJ/day) }\end{array}$} & \multirow{2}{*}{$\begin{array}{c}\text { Increase } \\
\text { in Storage } \\
\text { (MJ/day) }\end{array}$} & \multicolumn{3}{|c|}{ Storage Heat Delivered to: } & \multicolumn{3}{|c|}{ Storage Heat Losses * } \\
\hline & & & $\begin{array}{c}\text { DHW } \\
\text { (MJ/day) }\end{array}$ & $\begin{array}{l}\text { Heating } \\
\text { (MJ/day) }\end{array}$ & $\begin{array}{l}\text { Cooling } \\
\text { (MJ/day) }\end{array}$ & (MJ/day) & $(\mathrm{MJ} / \mathrm{hr})$ & $(B t u / h r)$ \\
\hline $1 / 01 / 75$ & 418.6 & 85.3 & 24.6 & 256.6 & 0.0 & 43.1 & 1.8 & 1890 \\
\hline $1 / 02 / 75$ & 0.0 & -346.4 & 0.0 & 302.3 & 0.0 & 44.1 & 1.8 & 1940 \\
\hline $1 / 05 / 75$ & 383.6 & 55.3 & 23.0 & 253.4 & 0.0 & 51.9 & 2.2 & 2280 \\
\hline $2 / 12 / 75$ & 332.4 & 105.4 & 66.0 & 111.4 & 0.0 & 49.6 & 2.1 & 2180 \\
\hline $2 / 19 / 75$ & 498.1 & 87.7 & 64.3 & 298.7 & 0.0 & 47.4 & 2.0 & 2080 \\
\hline $\begin{array}{l}\text { Winter } \\
\text { Average }\end{array}$ & & & & & & 47.2 & 2.0 & 2080 \\
\hline $7 / 10 / 75$ & 282.2 & 25.4 & 0.0 & 0.0 & 173.1 & 83.7 & 3.5 & 3680 \\
\hline $7 / 14 / 75$ & 63.2 & -106.9 & 0.0 & 0.0 & 77.1 & 93.0 & 3.9 & 4090 \\
\hline $.7 / 18 / 75$ & 161.6 & 76.7 & 0.0 & 0.0 & 9.4 & 75.5 & 3.1 & 3320 \\
\hline $7 / 21 / 75$ & 158.4 & -9.4 & 0.0 & 0.0 & 84.2 & 83.6 & 3.5 & 3680 \\
\hline $7 / 27 / 75$ & 298.8 & 205.1 & 0.0 & 0.0 & 0.0 & 93.7 & 3.9 & 4120 \\
\hline $\begin{array}{c}\text { Summer } \\
\text { Average }\end{array}$ & & & & & & 85.9 & 3.6 & 3780 \\
\hline
\end{tabular}

*Losses, computed by difference 
Table 23. Monthly and Annual Averages of Daily Mean Values (Heating Systems)

\begin{tabular}{|c|c|c|c|c|c|c|c|}
\hline & {$[1]$} & {$[2]$} & [3] & [4] & [5] & {$[6]$} & {$[7]$} \\
\hline Month & $\begin{array}{l}\text { Thermal } \\
\text { Storage } \\
\text { Temperature } \\
\left({ }^{\circ} \mathrm{C}\right)\end{array}$ & $\begin{array}{l}\text { Thermal } \\
\text { Storage } \\
\text { Capacity } \\
\text { (MJ) }\end{array}$ & $\begin{array}{l}\text { Change in } \\
\text { Thermal Storage } \\
\text { Capacity (daily) } \\
\text { (MJ/day) }\end{array}$ & $\begin{array}{l}\text { Thermal } \\
\text { Storage } \\
\text { Heat Balance } \\
(\mathrm{MJ} / \text { day }) \\
\end{array}$ & $\begin{array}{l}\text { Total Solar } \\
\text { Heat to } \\
\text { Loads } \\
\text { (MJ/day) }\end{array}$ & $\begin{array}{l}\text { Heating } \\
\text { System } \\
\text { Heat Losses } \\
(M J / \text { day })\end{array}$ & $\begin{array}{l}\text { Actual Solar } \\
\text { Heat to } \\
\text { Heating Load } \\
\text { (MJ/day) }\end{array}$ \\
\hline September & 69.5 & 874.3 & 8.5 & 240.6 & 153.7 & 86.9 & - \\
\hline October & .71 .3 & 905.9 & -6.5 & 185.6 & 111.3 & 74.3 & 84.6 \\
\hline November * & 62.6 & 751.4 & -1.4 & 202.0 & 146.2 & 55.8 & 172.0 \\
\hline December * & 49.9 & 526.8 & 0.8 & 223.7 & 170.8 & 52.9 & 222.0 \\
\hline January & 45.3 & 446.2 & 8.4 & 253.8 & 194.5 & 59.3 & 232.7 \\
\hline February & 48.4 & 501.2 & 20.9 & 292.5 & 236.0 & 56.5 & 244.8 \\
\hline March * & 54.9 & 615.2 & -29.0 & 251.3 & 191.3 & 60.0 & 142.7 \\
\hline April & 63.3 & 763.6 & 20.4 & 210.2 & 149.0 & 61.2 & 151.9 \\
\hline May & 64.4 & 733.4 & 17.8 & 165.0 & 82.3 & 82.7 & 97.3 \\
\hline June & 69.7 & 877.2 & 21.6 & 177.1 & 92.8 & 84.3 & - \\
\hline JuTy & 76.7 & 1001.7 & 5.5 & 223.4 & 130.5 & 92.9 & - \\
\hline August & 78.4 & 1030.0 & 4.4 & 209.8 & 122.0 & 87.8 & - \\
\hline $\begin{array}{l}\text { Annual } \\
\text { Average }\end{array}$ & 62.9 & 756.5 & & 219.6 & 148.4 & 77.2 & 168.5 \\
\hline
\end{tabular}

* Data for part of month only 


\begin{abstract}
Column [1] Thermal storage temperature is an average of three temperature probes inserted in the storage tank at the top, middle, and bottom

Column [2] Thermal storage capacity is the average heat capacity, based on the thermal storage temperature (above $20^{\circ} \mathrm{C}$, $68^{\circ} \mathrm{F}$ - room temperature) and the total volume of water in the tank (4275 liters, 1130 gallons)

Column [3] Change in thermal storage capacity is the daily change (midnight to midnight) of column [2]

Column [4] Thermal. storage heat balance is the difference between the total daily collected solar heat (see Table 16) and the change in thermal storage capacity (column [3])

Column [5]. Total solar heat to loads is the sum of all solar heat delivered to the domestic hot water, heating, and cooling loads

Column [6] Heating system heat losses is the difference between the thermal storage heat balance (column [4]) and the total solar heat delivered to load (column [5])

Column [7] "Actual" solar heat to heating load is the total of the solar heat delivered to the heating load (Table 16) and the thermal storage heat losses (column [6])
\end{abstract}

From Table 23 we can obtain a more complete picture of the magnitude of the thermal storage unit and associated equipment heat losses, where the correlation between the storage temperature and amount of heat loss is more apparent (note that we are assuming a constant building temperature of $20^{\circ} \mathrm{C}$, a condition that is not in fact constant-see Table 17). If we account for the variation in average daily building temperatures, we can obtain an annual average heat loss rate of:

$$
\begin{aligned}
& U_{L}=71.2 \mathrm{MJ} / \text { day }:\left(62.9^{\circ} \mathrm{C}-21.2^{\circ} \mathrm{C}\right) \\
& U_{L}=1.71 \mathrm{MJ} / \text { day } \cdot{ }^{\circ} \mathrm{C} .
\end{aligned}
$$

Column [7] of Table 23 accounts for this heat loss (for the months of October through May) by including it in the total solar heat contributed to load. This "actual" solar heat delivered to the cooling load thus 
increases the annual per cent solar heat delivered to the heating load to 87 per cent. 


\section{SYSTEM ECONOMIC ANALYSIS}

In the process of constructing the solar system and its installation in the house, detailed cost accounting was performed. The results are associated with the following applicable factors:

1. The unit is a custom design of comparatively small size;

2. In evaluating the necessary man-hours for repetitive tasks, the time requirement for the last units was used so that learning time would not be included,

3. University shop personnel, normality accustomed to new and unique work assignments, did most of the assembly and installation,

4. Tooling costs were minor and are not included, and

5. Extra costs are associated with the research aspects of the facilities, including redundancy in operating methods.

Since the cost of solar heating and cooling is the most important consideration in its adoption and practical use, there must be a clear understanding of criteria.

First, it is necessary to separate the cost of the solar components from the cost of the house itself. The cost of the house should be considered to include a conventional heating and cooling system, capable of meeting maximum denands. The solar energy system cost is then the additional costs of the solar components, such as the solar collector, thermal storage, associated piping, valves, etc. Not included are costs of furnace, distribution ducting, an air conditioner, water heater, and conventional controls because they would be used in a fuel-operated system, and they are also the conventional parts of the solar operated system. The air conditioner might be of a cheaper type in a conventional system, however. 


\section{TOTAL COST OF THE SOLAR PORTION OF THE SYSTEM}

Based on the criteria established in the above section, the total cost of the solar components (including installation) for CSU. Solar House $I$ is approximately $\$ 10,100.00$. This does not include the cost of the conventional components of the system (those necessary for a complete HVAC unit), the cost of distribution ductwork, and those costs associated with overhead and profit. This cost relates to the construction of the solar system, as depicted by Mode 1 (see Figure 7), and designed to meet 75 per cent of the space heating and cooling loads.

\section{LABOR COSTS}

Table 24 lists the labor time and costs associated with on-site fabrication and installation of the solar collectors (man-hours and crane time). The cost of labor for installing the rest of the solar. components is shown in Table 24 as $\$ 1,600.00$. The total labor cost for fabricating and installing the solar heating and cooling system (exclusive of the conventional, non-solar facilities) is therefore $\$ 3,684.00$. Labor for installing non-solar equipment is shown to total $\$ 400$.

\section{MATERIALS COSTS}

Tables 24 and 25 show the costs of materials in the solar collector $(\$ 3,022.00$ total $)$, and of the other solar components in the system $(\$ 3,400.00)$. The total solar material cost is therefore $\$ 6,422.00$. The total cost of conventional heating and cooling equipment (materials only) is $\$ 2,200.00$.

OPERATIONAL COSTS

Operational costs include the expense of natural gas for auxiliary energy and electricity for powering pumps and blowers. Table 26 shows the 
Table 24. Solar Collector Costs

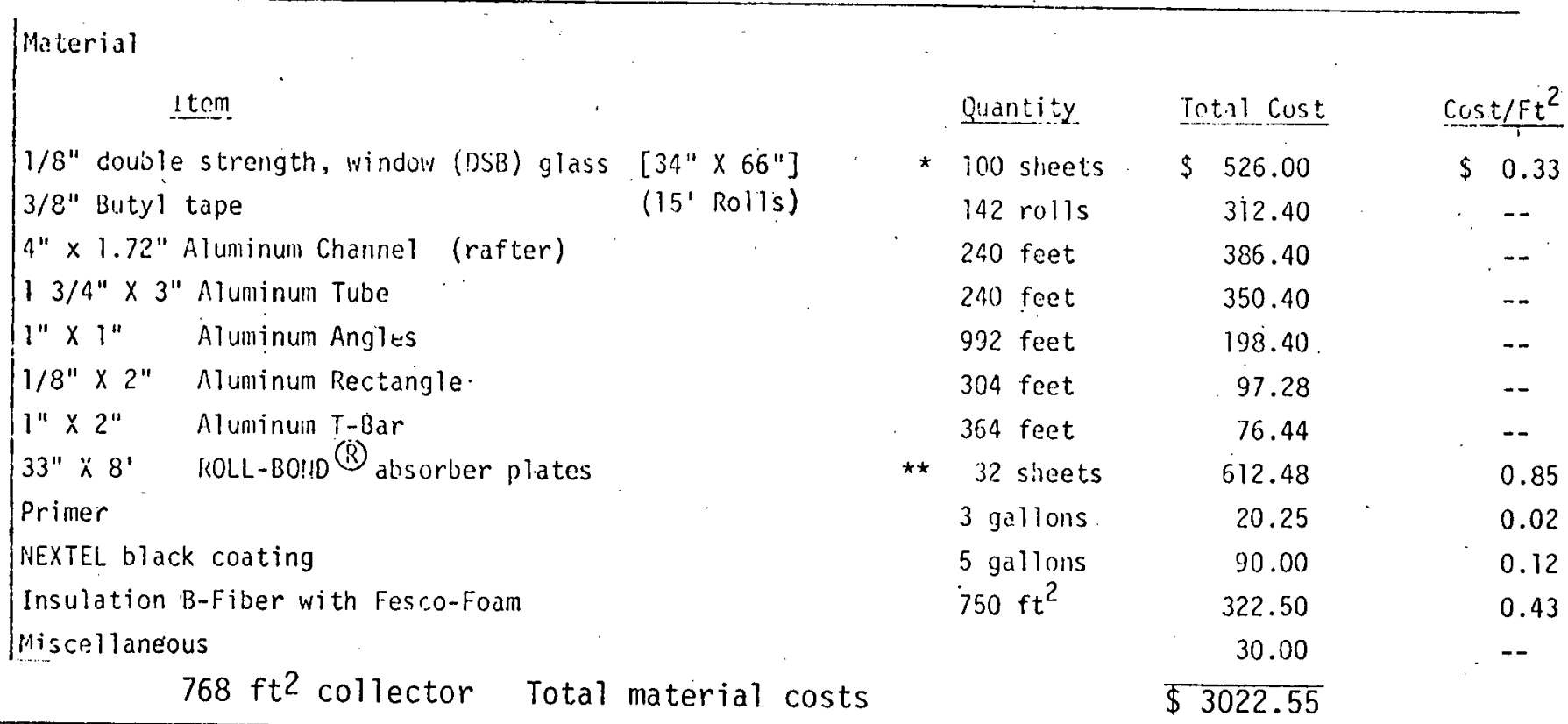

jabor

\section{Item.}

Cleaning Absorber Plate (32 a $1 / 4$ hours)

Pāinting Absorber Plate (32 a $1 / 2$ hours)

Baking Ausorber Plates (32 a $1 / 2$ hours)

Aluminum and Glass Frame Fabrication

Glass Preparation

Installing Aluminum Beams

installing lianifolde

Installing Insulation/Absorber Plate/Plumbing/

Glass Units for 16 Panels

[3 panels/day with 3 men]

Crane Tine

Miscellaneous (a $\$ 10 /$ hour) 44

Man-llours

3

16

16

18

6

23

20

128

44

34

318

Total labor costs
$\underline{\operatorname{Cos} t^{x: t *}}$

$\$ 48.00$

96.00

96.00

108.00

36.00

168.00

120.00

768.00

440.00

204.00

+ Includes shipping to job site

* Allows for $4 \%$ glass breakage

** Does not include design and set up charges

$\star * \star$ C $\$ 6.00 /$ hour 
Table 25. Solar Heating and Cooling System Costs

\begin{tabular}{|c|c|c|}
\hline \multicolumn{3}{|l|}{ "Solar" } \\
\hline Thermal storage tank with insulation & $\$ 350$ & \\
\hline Collector and exchanger pumps & 150 & \\
\hline Collector/storage heat exchanger & 400 & \\
\hline Preheat tank & 100 & \\
\hline Automatic by-pass valves & 200 & \\
\hline $\begin{array}{l}\text { Associated piping, valves, fittings, } \\
\text { insulation, etc. }\end{array}$ & 500 & \\
\hline $\begin{array}{l}\text { Extra cost of absorption cooling } \\
\text { equipment compared with conventional } \\
\text { vapor compression type }\end{array}$ & 1500 & \\
\hline Controls & 200 & \\
\hline Labor & 1600 & \\
\hline SUBTOTAL & $\$ 5000$ & 5000 \\
\hline \multicolumn{3}{|l|}{ "Conventional" } \\
\hline ITEM & $\cos T$ & \\
\hline Vapor compression air conditioner & $\$ 1100$ & \\
\hline Automatic valve & 100 & \\
\hline Hot water boiler & 500 & \\
\hline 40 gallon hot water heater & 100 & \\
\hline Circulating pump & 50 & \\
\hline Heating coils & 125 & \\
\hline Control instrumentation & 25 & \\
\hline Associated piping, valves, fittings, etc & 200 & \\
\hline Labor & 400 & \\
\hline SUBTOTNL & $\$ 2600$ & 2600 \\
\hline TOTAL & & $\$ 7600$ \\
\hline
\end{tabular}

* ARKLA 3-ton, lithium bromide cooling unit 2000 Cooling tower Cooling tower pump 
Table 26. Supplemental Ënergy Usage and Costs

(1 September 1974 to 31 August 1975)

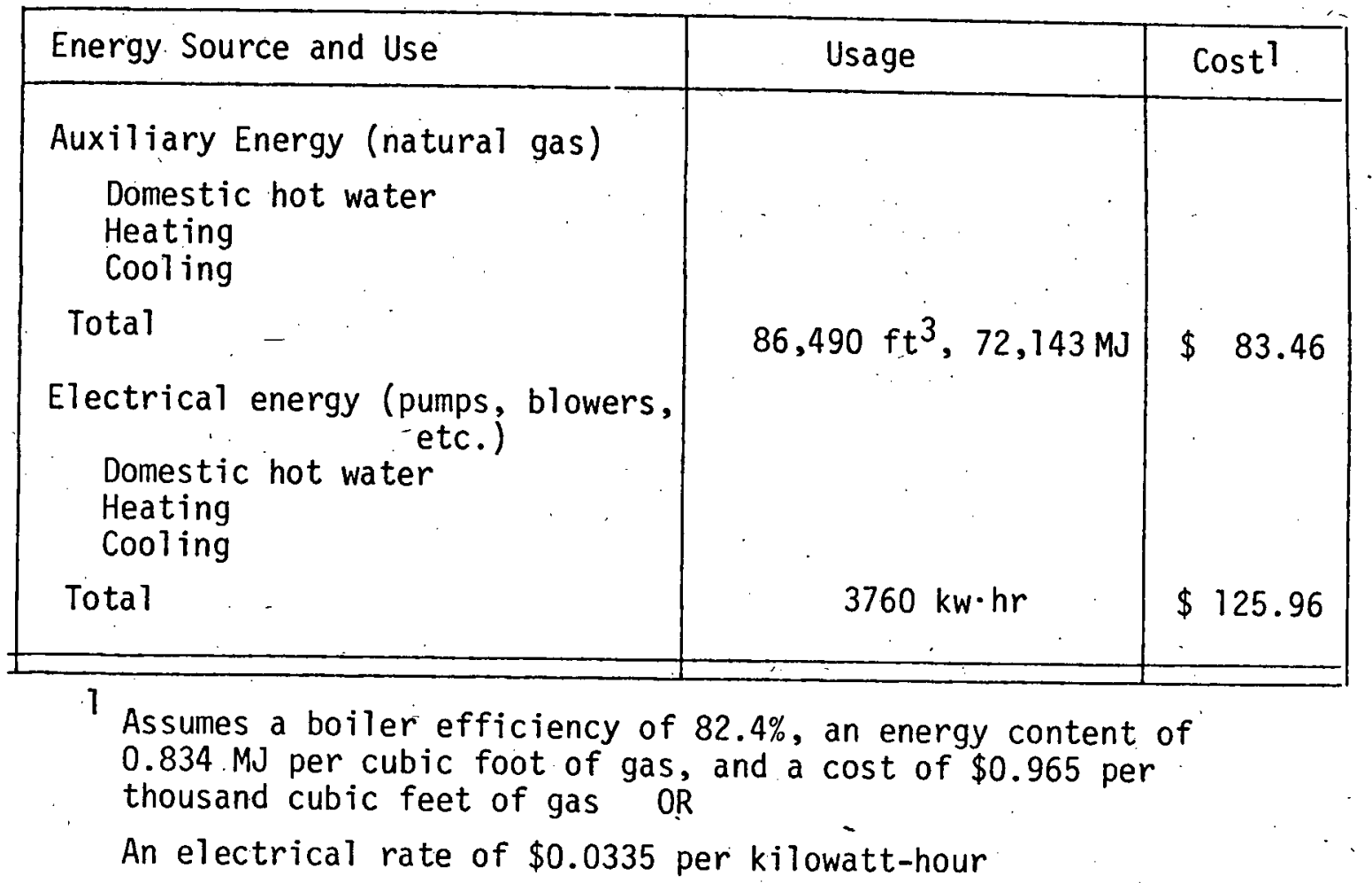


total energy usage and associated costs of supplemental energy during the period 1 September 1974 through 31 August 1975.

\section{LOCAL ECONOMIC FACTORS}

In the Fort Collins, Colorado region during the period 1 September 1974 to 31 August 1975, interest rates ranged from eight to nine per cent for home mortgages (new homes and remodeling of existing buildings), with amortization periods of 20 to 30 years. The local inflation rate averaged approximately eight per cent during the same period.

\section{EXPECTED SELLING PRICE OR COSTS}

The solar heating and cooling equipment at Colorado State University is part of an experimental system not expected to be sold in full or in part. But if it were commercialized in its present form, with collectors built on-site, and typical labor wage scales applied, along with customary overhead and profit, the total installed price of the system would be fifty to one hundred per cent higher than the expenditure shown above.

\section{MAINTENANCE FREQUENCY AND COSTS}

During the period from 1 September 1974 through 31 August 1975, required maintenance was limited to one service call on the lithium bromide absorption cooling unit. This service required six man-hours at a total cost of $\$ 86.00$. No collector maintenance was required, and non-experimental servicing of solar equipinent by laboratory personnel involved approximately 24 man-hours at a nominal value of $\$ 144.00$.

\section{USER REACTION AND COMMENTS}

Not applicable. 


\section{SUBSYSTEM PERFORMANCE}

SOLAR COLLECTORS

\section{Description of Physical Configuration}

The collector consists of sixteen 0.9 meter by 4.9 meter (three foot by sixteen foot) sections, for a total collector area of 71.3 square meters (768 square feet). The total exposed glass area is 65.7 square meters (707 square feet). Horizontal manifold pipes, $5 \mathrm{~cm}$ (2 inches) in diameter, at the top and bottom of the collector sections, run the length of the collector array (14.6 m, $48 \mathrm{ft})$.

Because of the experimental nature of the installation, and the need to replace the collector with new designs at a later date, a watertight sub-roof was made an integral part of the house design. This requirement imposed some restrictions on the collector design; e.g., the installation of a prefabricated modular unit becomes more difficult when the back side of the collector is unavailable for making plumbing connections. It was also desired to have a neat-appearing watertight solar collector capable of acting as a roof. These requirements further limited the possibilities of utilizing modular construction. The design finally developed is shown in Figure 16.

The collector is composed of aluminum structural supports, two sheets of B-quality double strength (1/8 inch) window glass, an aluminum absorber plate with internal tubes ("ROLL-BOND" R), and insulation beneath. The cover glasses are attached to the aluminum structure by a butyl tape and an aluminum cap strip. Approximately one-third of the glass covers have undergone an anti-reflection treatment (RCA "Magicote" process), courtesy of Honeywel1, Inc. As shown in Figure 17, the aluminum panel has a tube 


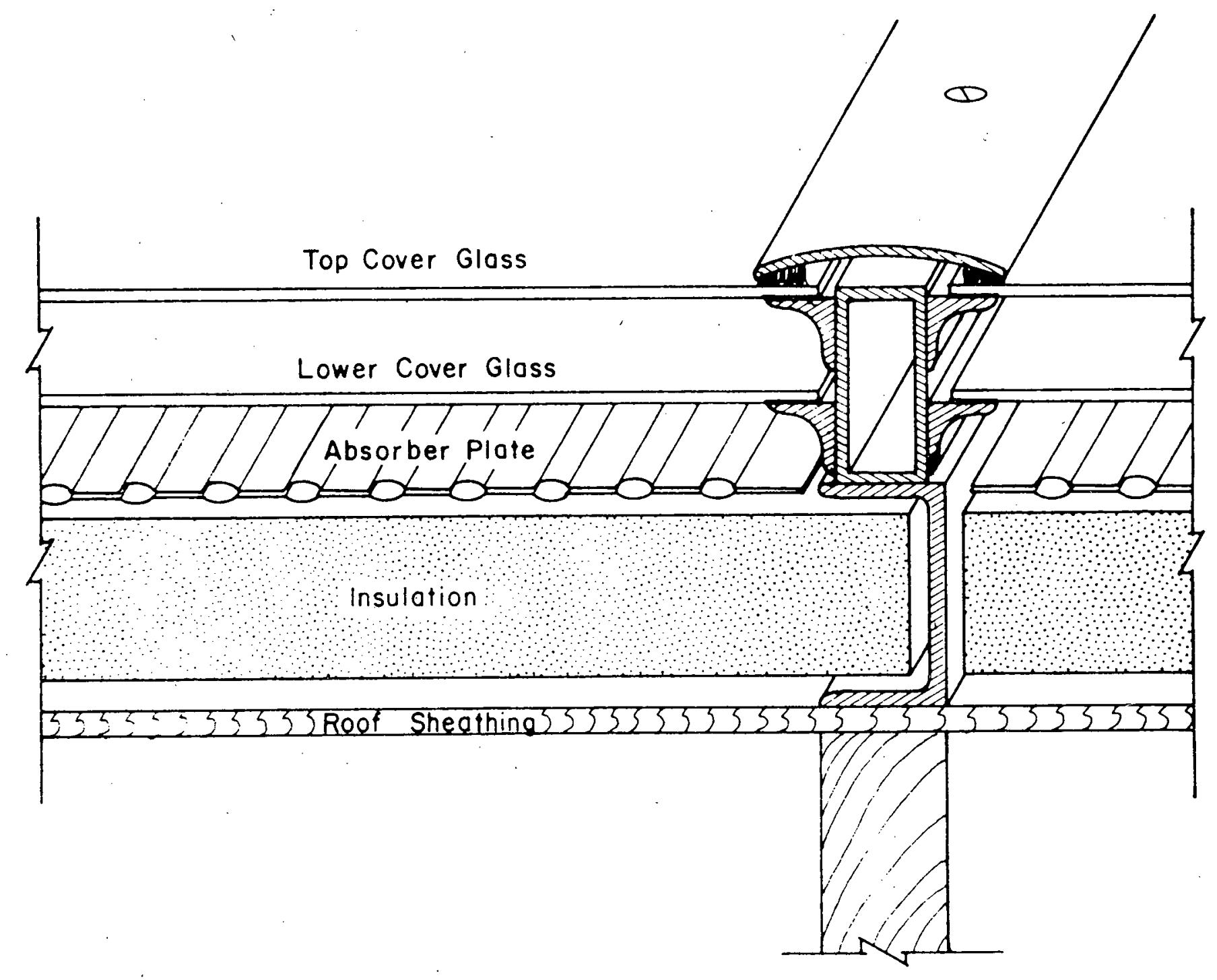

Figure 16

Solar Collector Cross Section 


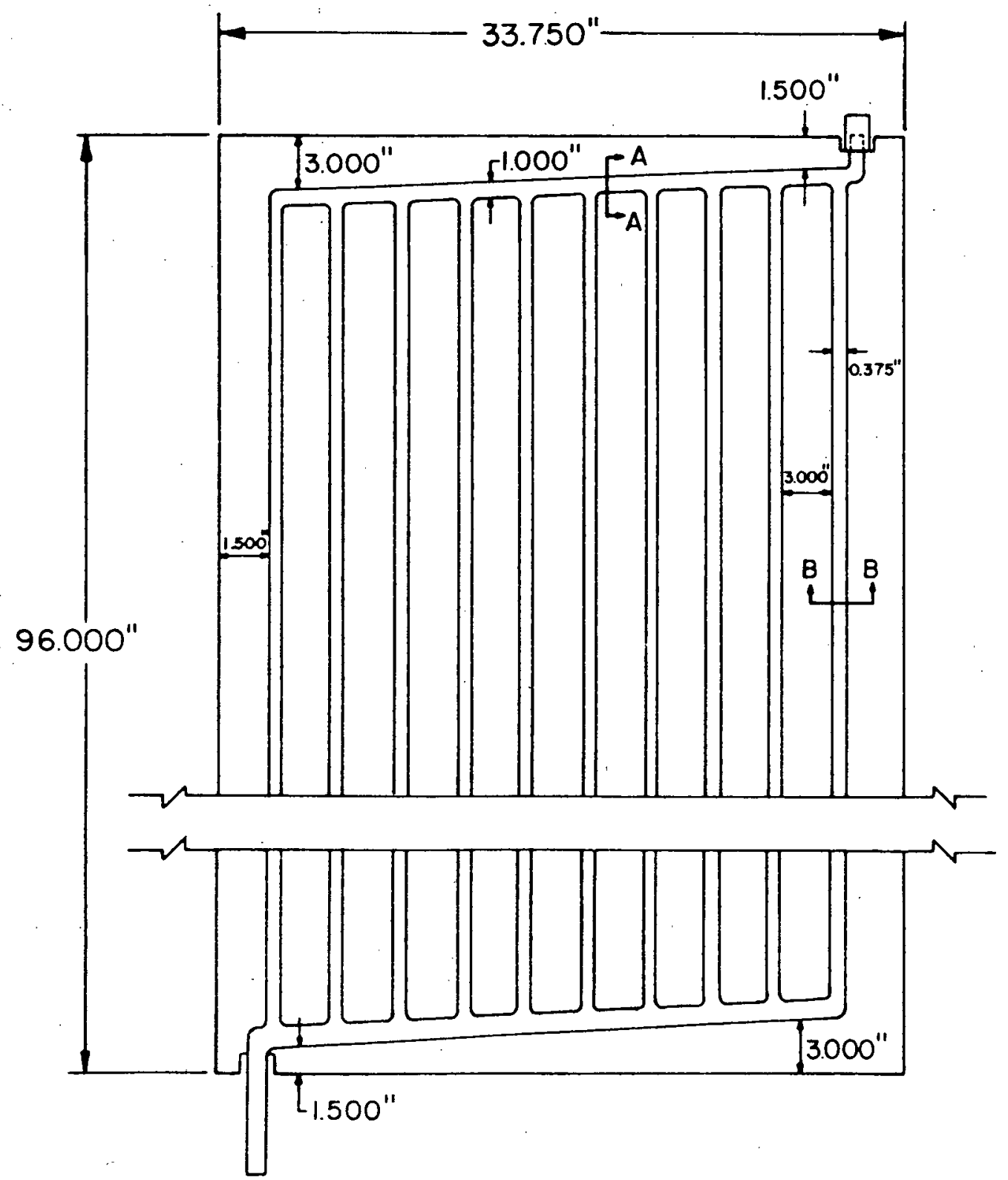

Figure 17

Absorber Panel Design

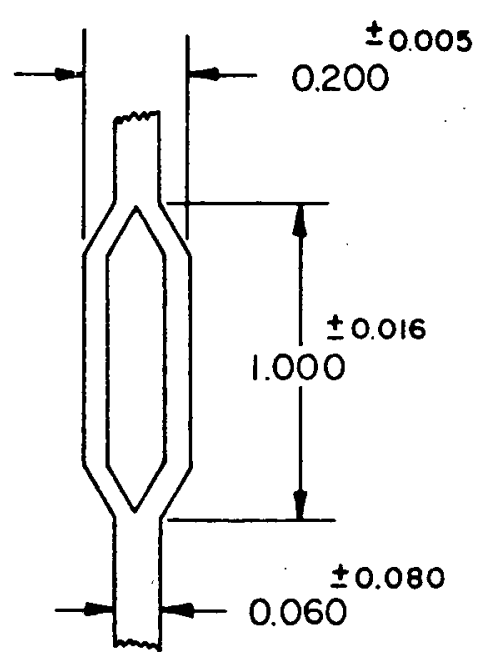

Section $A-A$

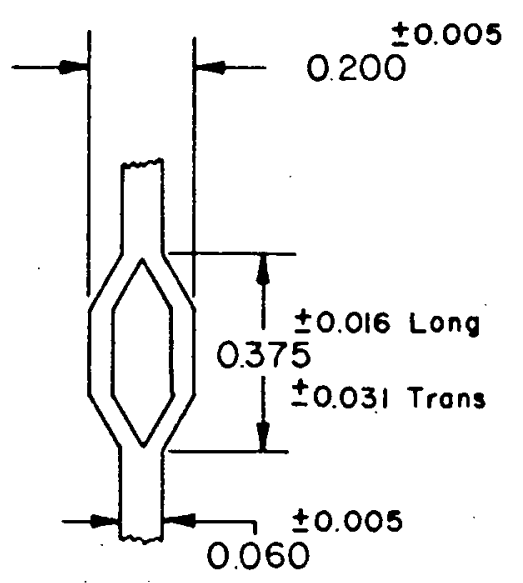

Section B-B 
pattern of parallel flow in multiple straight tubes between internal sloping manifolds. A flat-black "Nextel" $R$ acrylic coating was baked on the al uminum surface at a temperature of $200^{\circ} \mathrm{C}\left(400^{\circ} \mathrm{F}\right)$. The insulation consists of $2.54 \mathrm{~cm}$ (one inch) unbonded glass fiber mat on a $3.81 \mathrm{~cm}$ (1.5 inch) "Fesco-Foam" $R$ composite insulation. The overall insulating factor was computed to be $R=10.7 \frac{{ }^{\circ} \mathrm{Cm} 2}{\mathrm{~W}}\left(18.8 \mathrm{Hr}-\mathrm{ft}^{2}-{ }^{\circ} \mathrm{F} / \mathrm{Btu}\right)$.

Each sixteen foot section contains six sheets of glass and two absorber panels. The glass sheets measure $0.86 \mathrm{~m}$ (34 in) wide by $1.37 \mathrm{~m}(54 \mathrm{in})$ long, and are separately supported in the collector by a butyl caulking tape resting on an aluminum structural angle-iron. The absorber panels each measure 0.9 meters by 2.4 meters ( 3 feet by 8 feet), and are connected in series as shown in Figure 18.

A particular advantage of the ROLL-BOND aluminum absorber plate is its availability with preformed internal tubes. Thermal conductivity between the plate surface and the fluid circulated through the tubes is therefore maximized. In addition, there is an exceptional versatility in tube pattern design. Finally, panels were readily available at costs of $\$ 6.50$ to $\$ 8.50$ per square meter ( $\$ 0.60$ to $\$ 0.80$ per square foot) in 1974 .

Selection of a tube pattern (see Figures 17 and 18) is based on two concerns: drainage and rate of flow (which affect heat transfer rate and pressure drop). Provision for drainage of the solar collector during nondaylight hours can alleviate freezing problems and el iminate heat loss from the water in the collector at night.

The rate of flow was designed to produce a maximum temperature rise of $8^{\circ} \mathrm{C}\left(15^{\circ} \mathrm{F}\right)$ in the heat transfer fluid as it passes through the collector. At a solar radiation level of 950 watts / $\mathrm{m}^{2}\left(300 \mathrm{Btu} / \mathrm{hr}-\mathrm{ft}^{2}\right)$, and a solar collector efficiency of 40 per cent, this temperature increase is achieved at a flow rate of 3.781 iters/min $(1 \mathrm{gpm})$ per panel. Thus for all sixteen 


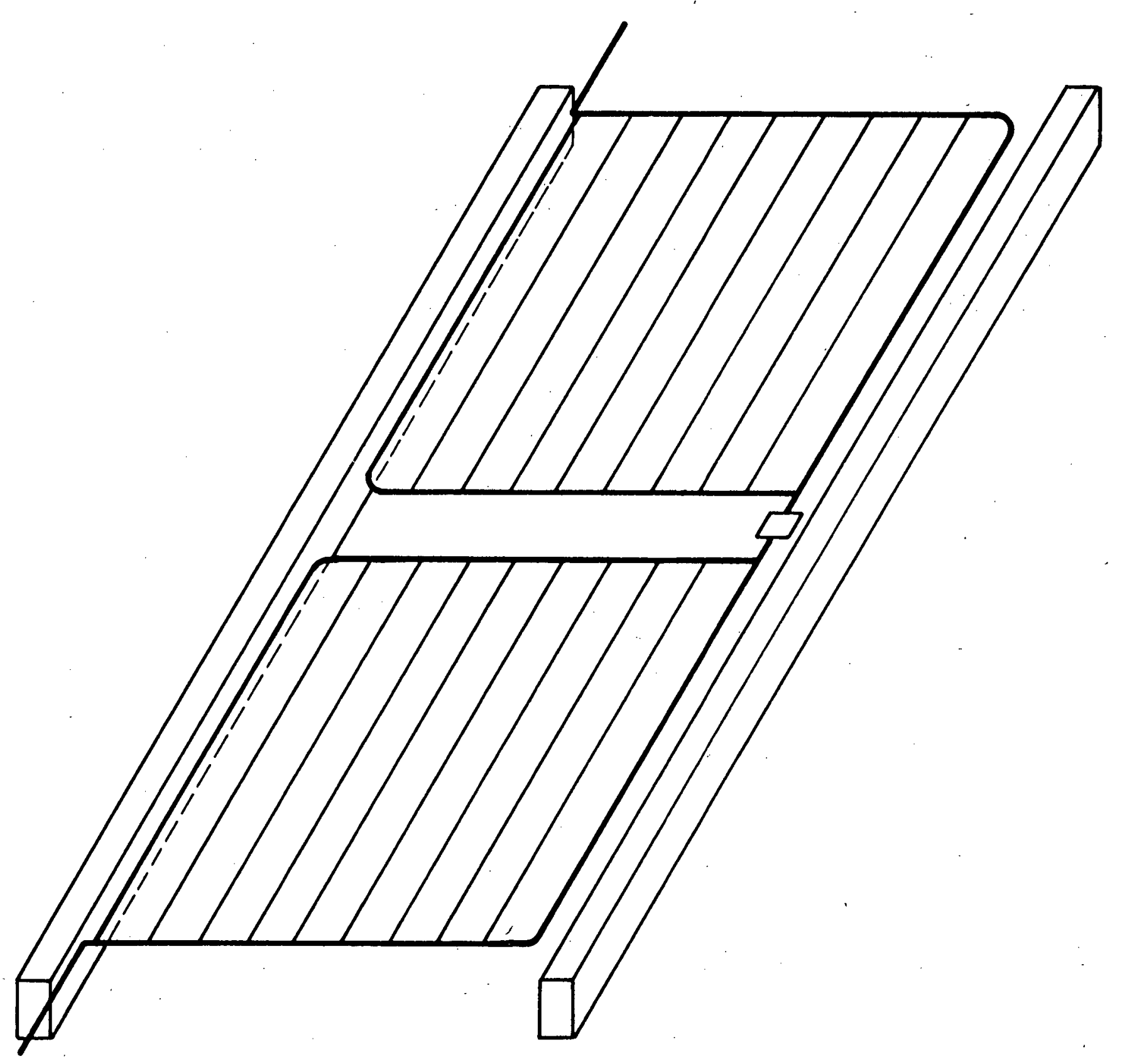

Figure 18

Collector Section Tube Pattern 
panels, the total flow to and from the collector is 60.51 iters/min (16 gpm).

The aluminum absorber plate is coated with a flat black, high absorptivity paint. The $3 M$ Nextel ${ }^{R}$ black coating has a total reflectance ( 3 to 35 microns) of less than two per cent. Application of the paint required a thorough cleaning of the absorber plate, the use of a primer, painting, and subsequent baking at $200^{\circ} \mathrm{C}\left(400^{\circ} \mathrm{F}\right)$ to prevent any possible off-gasing of the paint when the collector became operational.

Several panels of the Colorado State University solar collector contain glass treated by Honeywell, Inc. to reduce reflection losses (normally 8 per cent at normal incidence for glass with a refractive index of 1.5 ). Treatment was accomplished by a process originally developed by $R C A$, requiring dipping the glass in hydrofluosilicic acid. The surface should be durable inasmuch as glass treated 15 years ago by this process and exposed to the weather since then shows 96 per cent solar transmission.

of the sixteen sections in the solar collector, four contain glass which has been treated to reduce the reflection loss from 8 per cent to approximately 5 per cent. In addition, five sections contain glass that was partially treated (reducing reflection loss from 8 per cent to about 6 per cent). The remaining sections, with one exception, contain untreated glass. A special section contains two layers of glass having an infrared reflective coating on the interior surface of each piece of glass (supplied by Libbey-Owens-Ford). The purpose of the coating is to reflect the infrared reradiation from the absorber panel back to the black surface. During the period covered by this report, two layers of infrared reflective glass were used, but excessive solar absorption in the coatings showed the desirability of later substitution of ordinary glass for the top layer of treated glass. 
Experience has indicated that imperfections in the edge of the glass, such as small chips or rough cut surfaces, have a strong tendency to cause cracking and subsequent breaking in the interior glass sheets due to thermal stressing. Consequently, the edges of each sheet were sanded along the entire perimeter to remove these slight imperfections. In addition, glass that was chipped in shipping or handling was discarded. However, one panel was loaded with glass that contained some chipped edges as a comparison check.

Each sheet of glass was supported on all four edges by structural aluminum, and held in place by an adhesive butyl rubber tape. A primer improved adherence of the butyl tape to the aluminum. Adhesion to the glass, however, was insufficient to prevent creep of the interior glass at temperatures over $120^{\circ} \mathrm{C}\left(250^{\circ} \mathrm{F}\right)$. Glass movement in a test panel at a $45^{\circ}$ tilt amounted to about $20 \mathrm{~cm}$ ( 8 inches) over a period of three days. Small braces were therefore added to the cross bracing to prevent subsequent creeping.

While some of the structural aluminum was cut and preassembled in the shop, the main components of the collector were assembled on the subroof. Aluminum channels were first laid down and bolted to the subroof. Small teflon strips were inserted at each bolt connection to allow for differences in the thermal expansion between the aluminum collector and wood subroof. Each collector panel was then assembled one at a time. Insulation was first placed between the aluminum channels, and the absorber panels were then laid on the top surface of the insulation. Rectangular aluminum tubes with L-shaped ledges were then bolted to the channels. Plumbing connections between the panels and the manifolds were made. Adhesive butyl tape was attached to the aluminum structural components, and each sheet of glass was then set in place. Aluminum cap 
strips were then secured to the rectangular tubes, and with butyl tape between the glass and the strips, watertight closure was effected.

On-site collector construction involved high labor requirements. In addition to bad weather considerations, glass handling on a steep roof, working across a one-meter span on a partially completed panel, and the movement of tools and personnel to and from the subroof added considerably to the labor costs of the project. Prefabricated collectors (not available at start of project) are considerably more cost-effective.

One advantage of the particular installation method was the venting capability. As each of the collector sections was completed in place, its ends were left open. Natural convection through the panel thus prevented excessive temperatures in the collectors during installation. The ends were then closed following start-up of the collector pumping system.

\section{Thermal Performance Characteristics}

Collector Efficiency - Collector efficiency as a function of temperature and incident solar radiation was experimentaliy determined by operation of a collector test module. The results are shown in Figure 19. Efficiency is plotted against $\Delta T / H_{R}$, where $\Delta T$ is the difference between the temperature of the inlet water to the collector and the ambient air, and $H_{R}$ is the incident solar radiation on a $45^{\circ}$ tilted surface (normal to the collector). Wind speed was less than $\mathrm{l} \mathrm{Km} / \mathrm{hr}$. The efficiency is defined as the useful energy delivered divided by the incident solar radiation.

Typical operating conditions involve a temperature difference of $50^{\circ}$ to $55^{\circ} \mathrm{C}$ and an incident radiation of 750 to 900 watts $/ \mathrm{ml}^{2}$. These conditions provide an effective operating range of $\Delta T / H_{R}=35^{\circ} \mathrm{Cm}^{2} / \mathrm{Kw}$ to $\Delta T / H_{R}=$ $27^{\circ} \mathrm{C} \mathrm{m} / \mathrm{Kw}$, corresponding to a collector effiency of 27 to 35 per cent. However, this instantaneous collector efficiency is obtained under 


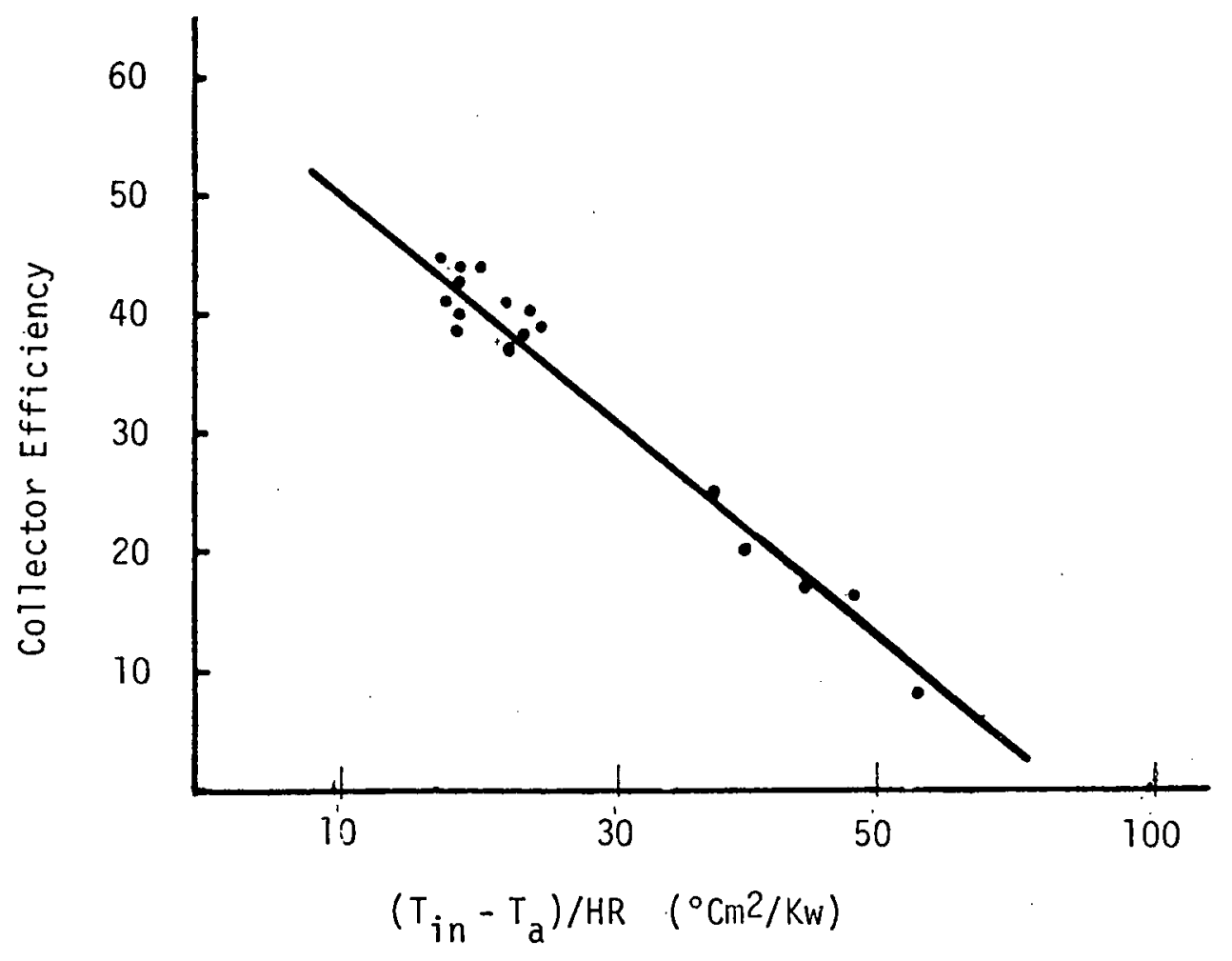

Figure 19. Collector Efficiency Versus $\triangle T / H R$ (Test Module) 
steady-state conditions and is not representative of typical operation in a house heating system. A more useful measure is the mean daily collector efficiency defined in two ways. ${ }^{n_{D}}$ is the total daily useful energy delivered to load (or thermal storage) divided by the total measured incident solar radiation during the full day, $H_{R} ; n_{D 0}$ is the total daily useful energy delivered to load (or thermal storage) divided by the incident solar radiation received during the operating hours of the collector.

Table 27 shows the mean monthly values of daily efficiency and the daily efficiency (during collector operations) for the period 1 September 1974 through 31 August 1975, as well as total daily solar radiation and radiation during collector operation. Although daily efficiency based on solar energy during operating hours was fairly constant, month-to-month, efficiency based on daily total radiation varies considerably. Most of the differences in the latter set of figures are due to variation in periods of collector operation, caused in turn by large variations in heat demand (very little energy is needed in April-May, and September-0ctober), and by seasonal changes in storage temperature, atmospheric temperature, and hours of sunshine.

Efficiencies of 30 to 35 per cent when operating, and 20 to 25 per cent based on total daily solar radiation, may appear lower than expected of a well-designed double-glazed collector. But these levels are satisfactory at the average temperatures of operation which usualiy prevailed. During the summer, mainly for air-conditioner operation, storage temperatures equaled or exceeded $80^{\circ} \mathrm{C}$ most of the time, so $\left(T_{\text {in }}-T_{\text {ambient }}\right)$ usually exceeded $60^{\circ} \mathrm{C}\left(108^{\circ} \mathrm{F}\right)$. The high percentage of seasonal heating load carried by solar (87 per cent) and the fact that 100 per cent of the load was met by solar until 18 December, were possible because of 
Table 27. Mean Monthly Averages of Daily Collector Efficiencies

\begin{tabular}{|c|c|c|c|c|c|c|}
\hline Month & Average ${ }^{n} D 0$ & $\begin{array}{l}{ }_{\text {Average } \mathrm{H}_{\mathrm{RO}}} \\
(\mathrm{MJ} / \text { day })^{3}\end{array}$ & $\begin{array}{c}\text { Average } \\
\text { Ambient } \mathrm{T}_{A O} \\
\left({ }^{\circ} \mathrm{C}\right)\end{array}$ & $\begin{array}{c}\text { Average } \\
\text { Storage } \mathrm{T}_{\mathrm{SO}} \\
\left({ }^{\circ} \mathrm{C}\right)\end{array}$ & ${ }^{2}$ Average $n_{D}$ & $\begin{array}{r}{ }^{2} \text { Average } H_{R} \\
(M J / d a y)^{3} \\
\end{array}$ \\
\hline September & 31.5 & 792 & 76.4 & 72.0 & 22.5 & 1106 \\
\hline October & -20.4 & 589 & 13.4 & 73.9 & 21.2 & 849 \\
\hline November & 31.2 & 490 & 3.7 & 63.6 & 19.6 & 780 \\
\hline December & 33.3 & 688 & 2.4 & 50.0 & 23.8 & 967 \\
\hline January & 33.0 & 745 & 2.9 & 46.3 & 24.6 & 999 \\
\hline February & 36.9 & 849 & 2.9 & 48.7 & 24.6 & 1274 \\
\hline March & 34.5 & 827 & 6.2 & 53.8 & 18.9 & .1395 \\
\hline April & 32.3 & 715 & 10.5 & 65.1 & 15.2 & 1513 \\
\hline May & 35.9 & 511 & 15.6 & 65.1 & 15.0 & 1217 \\
\hline June & 34.3 & 580 & 22.9 & 70.0 & 14.5 & 1375 \\
\hline July & 22.9 & 999 & 29.4 & 77.1 & 15.6 & 1474 \\
\hline August & 27.2 & 786 & 26.3 & 74.3 & 15.0 & 1429 \\
\hline
\end{tabular}

Includes solar radiation only when collector is operating

2 Includes days when collector did not operate at all

${ }^{3}$ Total MJ/day on a 71.3 square meter solar collector surface 
relatively high storage temperature (usually above $50^{\circ} \mathrm{C}$ ). Collector efficiency was 1 imited by, the elevated temperature, and adequate for the requirements. The self-regulating effect of temperature on efficiency is clearly evident - ample heat in storage causes a reduction in collection. efficiency because of high collector inlet temperature. This efficiency decrease is not a penalty, however, because it occurs when high collection is not needed and, in fact, not usable.

Pressure Drop Through Collectors - The pressure drop through the collectors was determined by calculation and by experimental confirmation of the pressure loss through a single absorber panel. Because of the varying flow rate through the collector (due to viscosity changes caused by temperature variations), the pressure drop varied over a considerable range. An equation was therefore developed, relating flow rate, pressure drop, density, and viscosity. Utilizing this calculated relationship, and the density and viscosity relationships of the collector fluid, the pressure drop due to friction across one absorber panel as a function of liquid type and liquid temperature was obtained. The correlation is shown graphically in Figure 20.

Thermal Loss Coefficient - Thermal loss coefficient as a function of the difference between the ambient and absorber plate temperature for no wind conditions was determined experimental1y to be: $U_{L}=4.2 \mathrm{w} / \mathrm{m}^{2} .{ }^{\circ} \mathrm{C}$, where the area is the overall dimensions of the collector and the temperature difference is the average plate temperature minus ambient temperature.

\section{Lifetime Performance Characteristics}

Corrosion - The metals and alloys in the solar collector liquid circuit consist of aluminum solar collector absorber plates, aluminum manifolds, copper pipe, a brass heat exchanger, and an iron-bronze pump. 


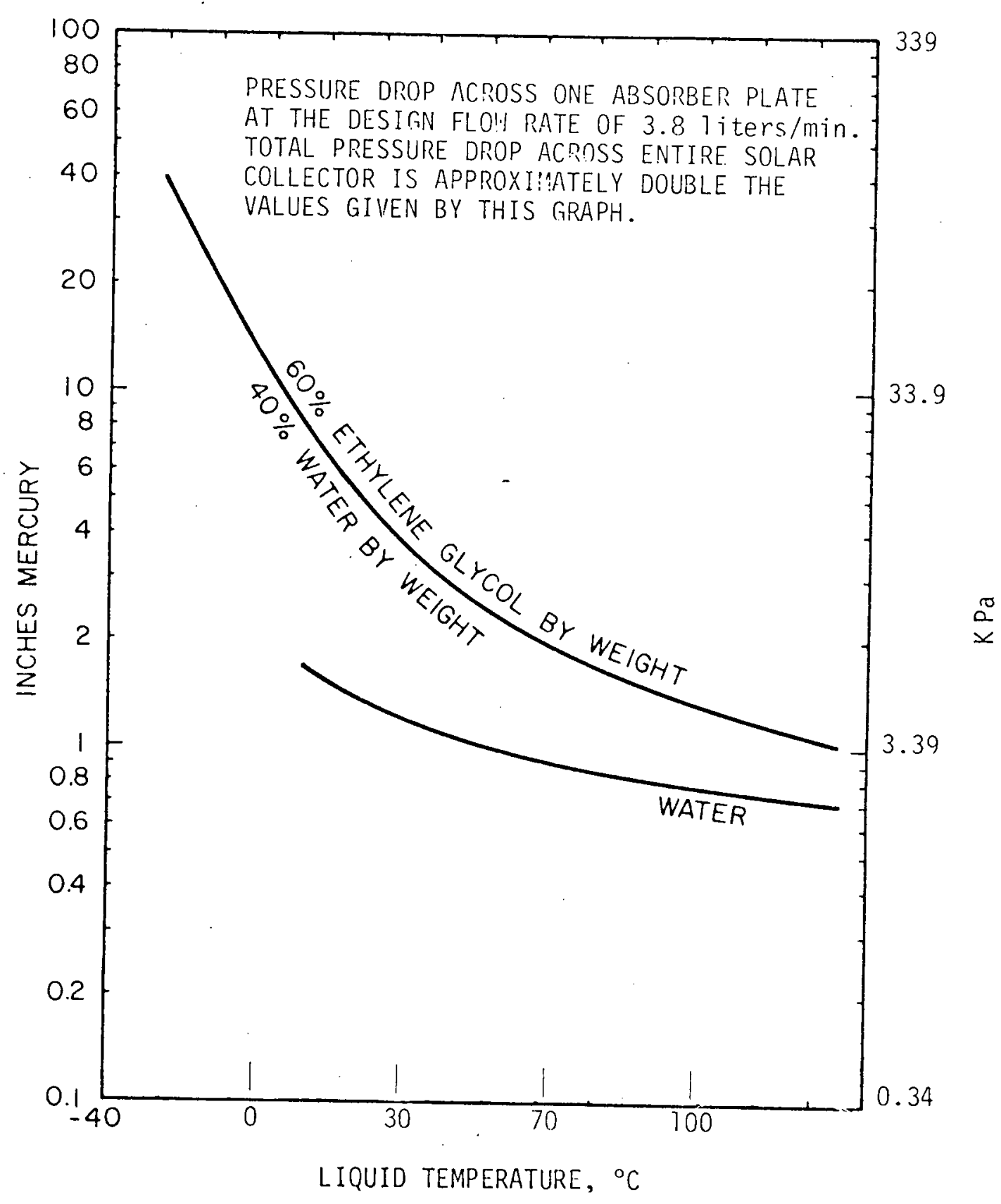

Figure 20. Pressure Drop Due to Friction Across One Alsorber Plate as a Function of Liquid Type and Liquid Temperature 
In order to reduce corrosion, the heat transfer fluid design velocity should be less than two to three feet per second. The CSU design velocity is about one foot per second.

The manufacturer of the ethylene glycol (antifreeze) recommends at least a 33 per cent concentration for protection against rust and corrosion. A corrosion inhibitor specially recommended by the manufacturer (Continental $0 i 1$ Company) for use in cooling systems containing aluminum is an additive to the ethylene glycol antifreeze solution. Sixty weight per cent of ethylene glycol (50 per cent by volume) affords the maximum antifreeze protection $\left(-52^{\circ} \mathrm{C}\right.$ or $\left.-62^{\circ} \mathrm{F}\right)$, and this concentration raises the boiling point by $10^{\circ} \mathrm{C}\left(18^{\circ} \mathrm{F}\right)$. Although a solution of less than 60 per cent concentration could have been used in the CSU solar collector loop with adequate antifreeze protection, it was judged desirable to have the higher boiling point possible with this concentration.

Dielectric Pipe Connections - To minimize the possibility of galvanic corrosion of the aluminum absorber panels, non-conducting, neoprene rubber radiator hose was used as connectors between absorber panels and aluminum manifolds and between manifolds and the copper piping in the collector liquid circuit. Hose clamps were used at all these connections.

Filtration - Aluminum can be severely pitted by galvanic corrosion in the vicinity of small metal particles (copper, brass, iron) that might be carried into the collector by the circulating liquid. To prevent this attack, all of the liquid passing through the collector loop is continuously filtered. The filter cartridges used removed particles as small as 50 microns, with a corresponding pressure drop of $14.5 \mathrm{KPa}$ [at a flow rate of the ethylene glycol-water mixture of 60.5 liters/minute, at a temperature of $\left.96^{\circ} \mathrm{C}\right]$. The pressure drop across the filter appears to vary inversely with the diameter of the smallest particle size removed. 
A fine filter cartridge was used first. While the flow rate appeared to be satisfactory initially, it eventually dropped to an unacceptably low value due to plugging. The material causing the plugging appeared to be impurities in the antifreeze used.

A medium filter cartridge was then installed and also performed satisfactorily for a time. After it became plugged, it was replaced by the coarse filter cartridge which has remained in service for over 9 months.

Getter - Heavy metal ions (copper, iron) that may be dissolved in the circulating fluid can react with aluminum by displacement of the aluminum and deposition of the other metal. In order to minimize this action in the collector, an ion "getter" is installed in the piping between the filter and the collector. The "getter" is a coil of aluminum window screen placed inside a $5.1 \mathrm{~cm}$ (2 inch) diameter radiator type rubber hose. The only metal between the getter and the aluminum solar absorber plates is aluminum. Reaction between dissolved copper ions and the fine aluminum wire is expected to provide protection for the collector.

Composition Monitoring - So that corrosive conditions, such as organic acids, can be prevented in the collector fluid, periodic chemical analysis of the solution is performed. Measurement of $\mathrm{pH}$, aluminum ion, and heavy metal ion content is made at suitable intervals. No hazardous conditions have been encountered, but several solution replacement have occurred for various reasons.

Corrosion Summary - In the total period of operation, no evidence of corrosion of the collector or any other components has been observed.

Leakage - There has been no apparent leakage of the collector heat transfer fluid from the collector array. : Slight cover glass leakage, however, has allowed rain and melted snow to enter the collector occasionaly, without damage. 
Hot Spots - Prevention of hot spots is accomplished by good flow distribution through the collector array and through the individual absorber plates. Quantitatively uniform flow is accomplished by ensuring that the head loss due to friction across the solar collector header and riser tubes $(\Delta H)$ is significantly greater than the total head loss in either header, top or bottom $(\Delta h)$, i.e., the ratio of $\Delta h / \Delta H$ should be small $(<0.1)$.

The value of $\Delta \mathrm{h} / \Delta \mathrm{H}$ for the CSU solar collector array is approximately 0.04 for a flow rate of one liter per second ( 16 gallons per minute) of water at a temperature of $95^{\circ} \mathrm{C}\left(203^{\circ} \mathrm{F}\right)$. The value of $\Delta \mathrm{h} / \Delta \mathrm{H}$ for a $60 \mathrm{per}$ cent ethylene giycol solution is less at the same temperature because $\Delta h$ will increase less than $\Delta H$. Calculations show that the flow in the middie riser will be 99 per cent of the flow in the two end risers, which is the maximum possible difference in flow rates between risers (the end risers will have the same and highest flow rates).

Experimental efforts show that the flow distribution in the CSU solar collector is quite uniform, with deviations of less than 0.5 per cent. The average outlet temperature for each section as $94^{\circ} \mathrm{C}$, with the greatest variation of the observed collector outlet temperature being $94.3^{\circ} \mathrm{C}$, indicating equal flow distribution in all risers.

\section{Flow Distribution in the Absorber Plate}

Experinents were conducted with the absorber plate in order to determine its flow distribution experimentally. NL a flow rate of 3.81 iters per minute (through one absorber plate, corresponding to the design flow rate of 60 liters/minute through the entire collector) at $81^{\circ} \mathrm{C}\left(178^{\circ} \mathrm{F}\right)$, the ratio of the head loss in one header $(\Delta h)$ divided by the total head loss across the absorber plate $(\Delta H)$ is about 0.1 . This implies that the driving head across the middle risers is about 91.7 per cent of the driving 
head across the end risers. Therefore, the flow rate through the middle riser is about 94 per cent of the flow rate through the end risers.

In addition to the above tests, a thermal infrared scanner ( $A G A$ Thermovision 680/102B) was used to photograph the temperatures of the entire absorber plate at the same time. Color photographs were made of the bottom, middle, and top (flow was from the bottom to the top; the absorber plate was mounted vertically) as $87^{\circ} \mathrm{C}\left(178^{\circ} \mathrm{F}\right), 60$ per cent ethylene glycol solution was pumped through the absorber plate at the rate of 3.8 liters/minute $(1 \mathrm{gpm})$. The photographs showed an equal flow distribution and, in addition, that the manifold was considerably hotter than the surrounding absorber plate area (including the risers), indicating an efficient energy collection by this manifold without excessive heat losses. The photographs thus confirmed the correctness of the hydraulic and thermal design of the absorber plate.

Breakage - Glass breakage during the installation of the collector involved only one plate out of a total of 96 installed. Breakage during the approximate two years of collector operation has been 1 imited to one lower glass cover, located in the middle section of the easternmost collector section. The breakage consists of one long crack which_has caused no noticeable difference in collector operating efficiency.

Stability of Collector Working Fluid - Periodic tests were conducted to identify possible corrosion, including measurements of pll and conductivity and an atomic absorption analysis of the collector fluid. These results are shown in Tables 28 and 29 . The initial high concentrations of zinc were probably due to the fact that the aluminum absorber panels had connections brazed onto the panels, and the brazing was the source of the zinc concentration. 
Table 28. $\mathrm{pH}$ and Conductivity Measurements

\begin{tabular}{|c|c|c|c|c|}
\hline Date & 23 October 1974 & 15 December 1974 & 5 May 1975 & $\begin{array}{c}\text { Control } \\
\text { Sample }\end{array}$ \\
\hline Temperature & $45^{\circ} \mathrm{C}$ & $24^{\circ} \mathrm{C}$ & $21^{\circ} \mathrm{C}$ & $23^{\circ} \mathrm{C}$ \\
\hline $\mathrm{pH}$ & 8.74 & 8.50 & 9.10 & 8.80 \\
\hline $\begin{array}{c}\text { Conductivity } \\
\text { (using K=0.1 } \\
\text { cel1) }\end{array}$ & $3600 \frac{\text { mhos }}{\mathrm{Cm}}$ & $2000 \frac{\text { mhos }}{\mathrm{Cm}}$ & - & $2500 \frac{\text { mhos }}{\mathrm{cm}}$ \\
\hline
\end{tabular}

Table 29. Atomic Absorption Analysis of Collector Fluids

\begin{tabular}{|c|c|c|c|c|}
\hline Sample & \multicolumn{4}{|c|}{ Results - Micrograms/liter of } \\
\hline \multirow{3}{*}{1} & Aluminum & Copper & Iron & Zinc \\
\cline { 2 - 5 } & 0.7 & 190 & 460 & 26 \\
2 & 0.3 & 44 & 110 & 870 \\
1* & - & - & 300 & 85 \\
$2 *$ & - & - & 150 & 237 \\
$3^{*}$ & - & - & 152 & 153 \\
\hline
\end{tabular}

Sample 1 is a new solution made up of $60 \%$ ethylene glycol and Fort collins tap water for collector control (2/6/75)

Sample 2 is a composite of five $100 \mathrm{ml}$ samples of collector fluid, taken over a period of 25 minutes $(2 / 6 / 75)$

Sample 3 (same as sample 2) $(3 / 6 / 75)$

*Additional analysis on $4 / 11 / 75$ 
HEAT TRANSFER SUBSYSTEM

Description of Physical Configuration

Working Fluids - Fluids in the heat transfer subsystem are an ethylene-glycol/water solution in the collector loop and water (with corrosion inhibitor) in the thermal storage unit, and heat exchangers (see section on Thermal Energy Storage Subsystem).

In the collector loop the heat transfer liquid is an aqueous solution of 60 per cent ethylene glycol, by weight.

Circulating Pumps - The circulating pumps are all 1750 RPM centrifugal type with direct-coupled motors. The hot water preheat and cooling tower pumps are of standard manufacture. The other pumps in the system were adapted to specific pressure (head) versus flow rate requirements. This was accomplished by using pumps slightly larger than needed and reducing (trimming) impeller diameters to obtain the exact specifications.

Heat Exchangérs - For 1iquid-to-1 iquid heat exchange without mixing of the liquids, shell-and-tube type heat exchangers were selected, with single-pass, counterflow design; the single-pass counterflow arrangement allows the temperature difference between filuids to be nearly constant along the exchanger. This feature results in a relatively small temperature loss across the exchanger. The collector heat exchanger is made up of two units in series because a single unit of sufficient length was unavailable.

The single-pass design involves multiple parallel tubes and a relatively high pumping rate to develop turbulent flow in the tubes. On the storage side of the collector heat exchanger, a flow rate of 95 liters per minute $(25 \mathrm{gpm})$ is obtainable with modest pump power because resistance in that loop is low. The high flow rate does, however, nearly eliminate temperature stratification in the storage tank. Highly stratified 
storage temperatures would thus come at the cost of a larger exchanger or a higher temperature loss.

Air Duct Coils - Solar heating requires a larger air duct coil surface than does auxiliary boiler heating. The increased size is due to the lower temperatures from solar storage $\left(65^{\circ} \mathrm{C}\right.$ design temperature down to as low as $25^{\circ} \mathrm{C}$ ) compared with the boiler water at $90^{\circ} \mathrm{C}$. The air duct coil size required for solar heating was thus selected and the same size coils were obtained for both solar and auxiliary because of easier installation and smaller air pressure loss with the identical coils. The coils consist of copper tubes with aluminum fins housed in a 50 by $56 \mathrm{~cm}$ ( 20 by 22 inch) duct section.

\section{Other System Components}

Domestic Hot Water and Solar Preheat Tank - An automatic gas-fired hot water heater acts as the auxiliary heater for domestic hot water. It is a 40 gallon (150 liter) glass lined tank with 35 gallon per hour recovery rate at $100^{\circ} \mathrm{F}$ temperature rise $\left(1321\right.$ iters/hour at $\left.55^{\circ} \mathrm{C} . \Delta \mathrm{T}\right)$. The rated output is $39.8 \mathrm{MJ} / \mathrm{hr}(42,000 \mathrm{Btu} / \mathrm{hr})$.

The hot water solar preheat tank is a standard insulated 80 gallon (300 liter) glass lined electric hot water heater with the heating element and control non-operational.

Auxiliary Hot Water Boiler - The hot water boiler is a stock manufacturid unit for domestic hot water heating systems. It was selected to provide the full heating and cooling requirements of the building when no solar heat is in storage. The boiler is gas-fired with a rated output of $85.3 \mathrm{MJ} / \mathrm{hr}(90,000 \mathrm{Btu} / \mathrm{hr})$ and output of $68.2 \mathrm{MJ} / \mathrm{hr}(72,000 \mathrm{Btu} / \mathrm{hr})$. At the 1585 meters (5200 feet) altitude of Fort Collins, corresponding ratings are $68.2 \mathrm{MJ} / \mathrm{hr}(72,000 \mathrm{Btu} / \mathrm{hr})$ and $54.6 \mathrm{MJ} / \mathrm{hr}(57,600 \mathrm{Btu} / \mathrm{hr})$, respectively. 
Heating System Performance

Heat losses from the solar components during the heating season contribute to the supply of heat for the building. Thus the solar contribution to the heating load (space heating and hot water) is equal to the useful energy delivered from the solar collector to the thermal storage unit. Tables 4 through 16 show the solar and auxiliary heat supplied for space heating during the period 1 September 1974 to 31 August 1975. Prior to 18 December 1974, solar provided 100 per cent of the heating load. Gas usage is seen to be primarily during periods of high heat demand, and only on twenty-three days did it have to supply more than half the total requirements. There were no days during which fuel supplied the full load.

An important influence on the auxiliary energy required by the heating and cooling system is exerted by the operating characteristics of the auxiliary gas boiler. Previously the control instrumentation required the maintenance of a preset temperature in the boiler. Gas required simply for this temperature maintenance can be a significant portion of the total usage because of limited fuel heat demand (auxiliary heat was required only 20 days out of 123 during the period 1 October 1974 to 31 January 1975). The principal heat loss from the boiler is to air continually rising through the hot boiler tubes to the stack and outdoor atmosphere.

The boiler in CSU Solar House I was found to require, for temperature maintenance only, 3400 liters (120 cubic feet) of gas per day when thermostatted at $38^{\circ} \mathrm{C}\left(190^{\circ} \mathrm{F}\right)$ (cooling season) and 2300 liters (80 cubic feet) of gas per day at a setting of $65^{\circ} \mathrm{C}\left(150^{\circ} \mathrm{F}\right)$ (heating season). During the month of August the requirements of a "hot boiler" accounted for more than 5 per cent of the total gas volume utilized. In the first half of the heating season, the hot. boiler condition required $6330 \mathrm{MJ}$ out of a total 
gas usage of $9480 \mathrm{MJ}$; i.e., two-thirds of the gas usage was for maintaining boiler temperature.

THERMAL ENERGY STORAGE SUBSYSTEM

Description of Physical Configuration

The thermal energy storage system consists of 4275 liters of water in a steel tank. The storage tank was fabricated from 16 gauge $(1.5 \mathrm{~mm}) \mathrm{gal-}$ vanized sheet steel. Seams and pipe connections to the tank are arc welded. A $60 \mathrm{~cm}$ (24 inch) diameter manhole in the top of the tank allows complete access to the inside. Each piping connection on the tank is provided with a shut-off valve and a neoprene hose connection to the copper piping. The tank is electrically isolated from all other plumbing components to prevent electrolytic corrosion of the tank.

The specification of the storage tank are:

$\begin{array}{lcc}\text { Diameter } & 1.67 \text { meters } & \text { (5.5 feet) } \\ \text { Height } & 1.82 \text { meters } & (6.0 \text { feet }) \\ \begin{array}{l}\text { Height of top cone } \\ \text { section }\end{array} & .27 \text { meters } & (0.9 \text { feet }) \\ \text { Volume } & 4275 \text { liters } & \text { (1131 U.S. gallons) } \\ \text { Weight empty } & 213 \text { kilograms } & 470 \text { pounds } \\ \text { Weight filled } & 4374 \text { kilograms } & 9644 \text { pounds }\end{array}$

Corrosion Control - Table 30 shows the composition of the corrosion inhibitor used. When added to distilled and/or demineralized water, the resulting $\mathrm{pH}$ of the aqueous solution should be between 7.5 and 8.0 .

Insulation on the storage tank consists of two layers of bonded glass fiber double-faced batt insulation having an $R$ factor of $17.7 \frac{{ }^{\circ} \mathrm{Cm}}{\mathrm{W}}$ $\left(30.4 \frac{\mathrm{Hr}-{ }^{\circ} \mathrm{F}-\mathrm{ft} 2}{\mathrm{Btu}}\right)$. Measured heat loss greatly exceeded this specified 
Table 30. Compositon of Corrosion Innibitor

\begin{tabular}{|lc|}
\hline \multicolumn{1}{|c}{ Ingredient } & $\begin{array}{c}\text { Per Cent } \\
\text { by Weight }\end{array}$ \\
\hline $\begin{array}{l}\text { Mercaptobenzothiazole } \\
\text { (technical grade, } 92 \text { per cent min) }\end{array}$ & 15.1 \\
$\begin{array}{l}\text { Sodium borate decahydrate, } \\
\mathrm{Na}_{2} \mathrm{~B}_{4} \mathrm{O}_{7} \cdot 1 \mathrm{OH}_{2} \mathrm{O}\end{array}$ \\
$\begin{array}{l}\mathrm{Disodium} \text { phosphate anhydrous } \\
\mathrm{Na}_{2} \mathrm{H} \mathrm{PO}_{4}\end{array}$ \\
\end{tabular}

rating, probably because of air circulation between insulation and the tank wall. Subsequent to the period of this report, the glass fiber batts were replaced by a sprayed-on cellulose insulation.

\section{Thermal Performance Characteristics}

The storage unit is located in the heated basement of the building where there is a mean temperature of the immediate surroundings of $20^{\circ}$ to $22^{\circ} \mathrm{C}\left(68^{\circ}-72^{\circ} \mathrm{F}\right)$ during the entire year. Thermal losses are therefore dependent almost entirely upon the storage tank temperature. These losses are discussed in the section on Energy and Mass Balances.

The storage capacity is 4275 liters of water. For a temperature range of $25^{\circ} \mathrm{C}$ to $95^{\circ} \mathrm{F}\left(78^{\circ} \mathrm{F}\right.$ to $\left.203^{\circ} \mathrm{F}\right)$, storage of $1090 \mathrm{MJ}\left(1.15 \times 10^{6} \mathrm{Btu}\right)$ is provided. The storage tank must operate at local atilospheric pressure, so the temperature is limited by the local boiling point of water $\left(95^{\circ} \mathrm{C}\right.$ $\left.203^{\circ} \mathrm{F}\right)$. 
AIR CONDITIONING SUBSYSTEM

\section{Description of Physical Configuration}

The cooling subsystem selected for CSU Solar House I is an. ARKLA-Servel 3-ton, lithium bromide absorption air-conditioner, modified to utilize hot water (instead of natural gas) as the heat supply to the generator. The ARKLA gas-fired unit was initially rated at 3.5 tons $(39.8 \mathrm{MJ} / \mathrm{hr})$. With solar heat supply, it is rated at 3 tons, $34 \mathrm{MJ} / \mathrm{hr}$. A forced draft cooling tower is employed for cooling the water circulated through the condenser and absorber of the air conditioner.

Heat Rejection Method - In order to match the capacity of the ARKLA air conditioner, the cooling tower must have a capacity of about $85 \mathrm{MJ} / \mathrm{hr}$ $(90,000 \mathrm{Btu} / \mathrm{hr})$ with a $6^{\circ} \mathrm{C}\left(10^{\circ} \mathrm{F}\right)$ approach and a $10^{\circ} \mathrm{C}\left(18^{\circ} \mathrm{F}\right)$ range.

The unit chosen is a galvanized steel, asbestos-packed cooling tower with a nominal capacity of 8 tons $(91 \mathrm{MJ} / \mathrm{hr} ; 96,000 \mathrm{Btu} / \mathrm{hr})$. The air fan is driven by a $.45 \mathrm{kw}(1 / 3$ horsepower) electric motor. The tower is designed for water flow rates of 1.0 to $1.7 \mathrm{l} / \mathrm{sec}(16$ to $27 \mathrm{gpm}$ ).

A Penn Aquastat electric fan control permits operation of the cooling tower fan only when required for a preset discharge water temperature. There are two conditions, both of which must be met, for the fan to operate. First, the ARKLA unit must be running, and second, the water temperature from the tower must be at least $24^{\circ} \mathrm{C}\left(75^{\circ} \mathrm{F}\right)$.

Thermal Performance Characteristics

Design Characteristics - The generator in a gas-fired ARKLA machine was replaced at the factory with one supplied by hot water entering at the bottom. With $38 \mathrm{l} / \mathrm{min}(10 \mathrm{gpm})$ of cooling water at $24^{\circ} \mathrm{C}\left(75^{\circ} \mathrm{F}\right)$, a hot water supply of $42 \mathrm{~T} / \mathrm{min}(11 \mathrm{gpm})$ at $87^{\circ} \mathrm{C}\left(188^{\circ} \mathrm{F}\right)$ will provide the full 
3-ton capacity $(34.1 \mathrm{MJ} / \mathrm{hr})$. At lower hot water temperatures, cooling capacity is lower, reaching 77 per cent at about $81^{\circ} \mathrm{C}\left(178^{\circ} \mathrm{F}\right)$ : Design temperature limits for hot water supply are $80^{\circ}$ to $95^{\circ} \mathrm{C}\left(175^{\circ}\right.$ to $\left.202^{\circ} \mathrm{F}\right)$. The temperature inside the evaporator is in the range of $3^{\circ}$ to $10^{\circ} \mathrm{C}\left(35^{\circ}\right.$ to $50^{\circ} \mathrm{F}$ ) (direct expansion) with a normal operating temperature of $7^{\circ} \mathrm{C}$ $\left(45^{\circ} \mathrm{F}\right)$. Performance data are presented in Figure 21 . Operation at a temperature as $10 \mathrm{w}$ as $80^{\circ} \mathrm{C}$ is possible at this location because of a dependable cooling water temperature of $24^{\circ} \mathrm{C}$ rather than a usual cooling water design temperature of $30^{\circ} \mathrm{C}$. While lower cooling water temperature permits a lower generator temperature, cooling capacity is not appreciably affected.

Because of cooling water temperature typically about $5^{\circ} \mathrm{C}\left(10^{\circ} \mathrm{F}\right)$ lower than even the reduced $24^{\circ} \mathrm{C}\left(75^{\circ} \mathrm{F}\right)$ design level, heat supply temperatures can be about $3^{\circ} \mathrm{C}\left(6^{\circ} \mathrm{F}\right)$ lower than usual. For effective operation at, these conditions, the concentration of the lithium bromide solution has been reduced to 51 to 54 per cent, from the customary 54 to 57 per cent. Temperature-concentration diagrams show that crystallization cannot occur in the absorber or in the generator provided that generator input temperature does not fall below $80^{\circ} \mathrm{C}\left(175^{\circ} \mathrm{F}\right)$. Below this temperature vapor formation occurs at a rate too low for effective lifting of the LiBr solution in the bubble pump, so after a time, the concentration in the generator could increase to the crystallization point. The generator is therefore always provided a heat supply at a temperalure no lower than $80^{\circ} \mathrm{C} \cdot\left(175^{\circ} \mathrm{F}\right)$

The unit has been modified also by the installation of a diverter valve for the cooling water to the absorber. The valve will divert (directly to the condenser) approximately 50 per cent of the cooling water when its temperature falls below $18.3^{\circ} \mathrm{C}\left(65^{\circ} \mathrm{F}\right)$ : The valve remains 


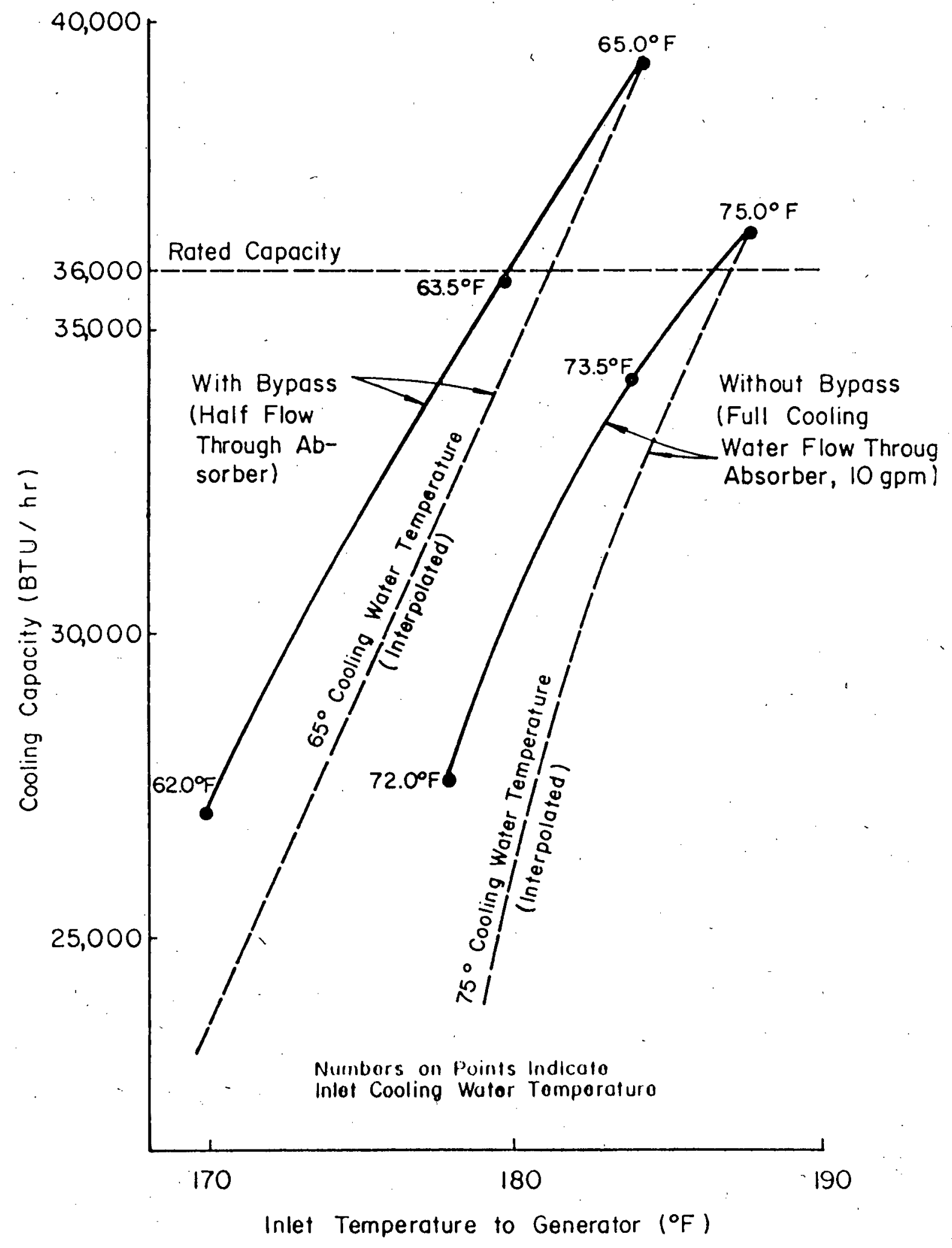

Figure 21. Measured Performance of ARKLA 3-ton Lithium Bromide Absorption Cooler 
open until the cooling water temperature rises to $20.5^{\circ} \mathrm{C}\left(69^{\circ} \mathrm{F}\right)$, giving an effective dead band of $2.2^{\circ} \mathrm{C}\left(4^{\circ} \mathrm{F}\right)$. The effect of the bypass valve is an increase in the capacity of the cooling unit by approximately $2.8 \mathrm{MJ} / \mathrm{hr}(3000 \mathrm{Btu} / \mathrm{hr})$ at the design generator temperatures. However, this advantage is lost when the input temperature to the generator is near its lower limit.

These modifications permit advantage to be taken of prevailing low wet bulb temperatures and, consequently, allow an increase in capacity over most of the range of generator temperatures. The location of the Colorado State University solar house at 1585 meters (5200 feet) elevation puts an upper 7 imit of $95^{\circ} \mathrm{C}\left(202^{\circ} \mathrm{F}\right.$ ) on hot water temperature (local boiling point) because a non-pressurized system is used.

As the air cooling capacity of the unit decreases at lower generator temperatures, dehumidification performance also declines. Operating at full capacity, the refrigerant finishes vaporizing at Point $B$ (see Figure 22), thereby cooling the entire evaporator surface on which moisture condenses. At a lower generator temperature, the reduced quantity of the refrigerant finishes vaporization at a position such as Point $c$, so the condensate covers only the upper portion of the finned area. Air temperature reduction is then about 77 per cent of the full capacity, but about 10 per cent of the condensate formed on the upper portion of the finned area will re-evaporate as it flows down over the lower, uncooled area. The net result is an air cooling capacity of 77 per cent and a dehumidification capacity of about 65 per cent of rating.

Experimental Characteristics - Figure 23 shows the quantitites of solar heat and auxiliary heat delivered to the cooling unit during the month of August, 1974. 


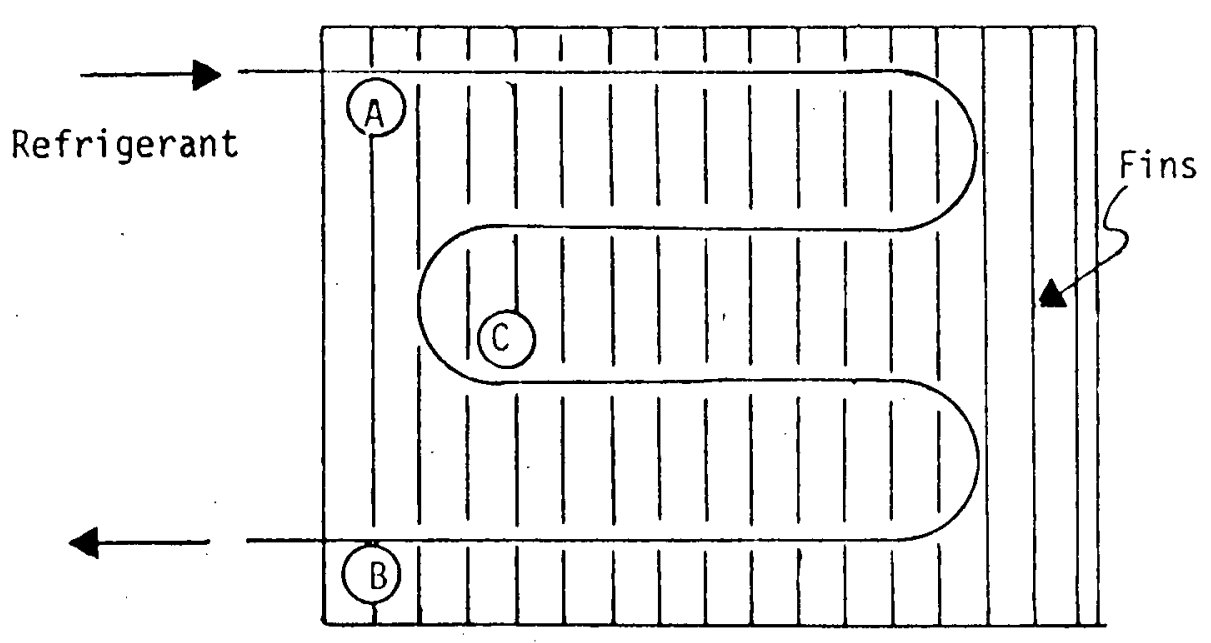

Figure 22. Evaporator Schematic

The cooling load of CSU Solar House $I$ is not representative of typical home cooling loads because of the large number of people occupying and visiting the building. Approximately 3,000 persons visited the House during the month of August, so the percentage of this unusual cooling load carried by solar is of less significance than the quantity of solar energy provided for cooling purposes. Even more important is the cooling load carried by solar under various control procedures.

During the period 1-10 August, a two-stage control was utilized for selecting solar or auxiliary to meet the cooling load. One contact in the thermostat provided cooling by solar whenever the temperature in the building rose above $22^{\circ} \mathrm{C}\left(71.6^{\circ} \mathrm{F}\right)$ and the temperature of thermal storage exceeded $82^{\circ} \mathrm{C}\left(180^{\circ} \mathrm{F}\right)$. If the cooling demiand could not be met, and the house temperature continued to rise above $23.4^{\prime \prime} \mathrm{C}\left(74.7^{\circ} \mathrm{F}\right)$, fuel was then used to supply hot water to the air conditioner so that the full cooling load could be met even though the storage temperature exceeded the minimum required inlet temperature to the generator of the cooling unit.

This control system was modified on 10 August to provide solar cooling whenever the thermal storage temperature exceeded the minimum generator 


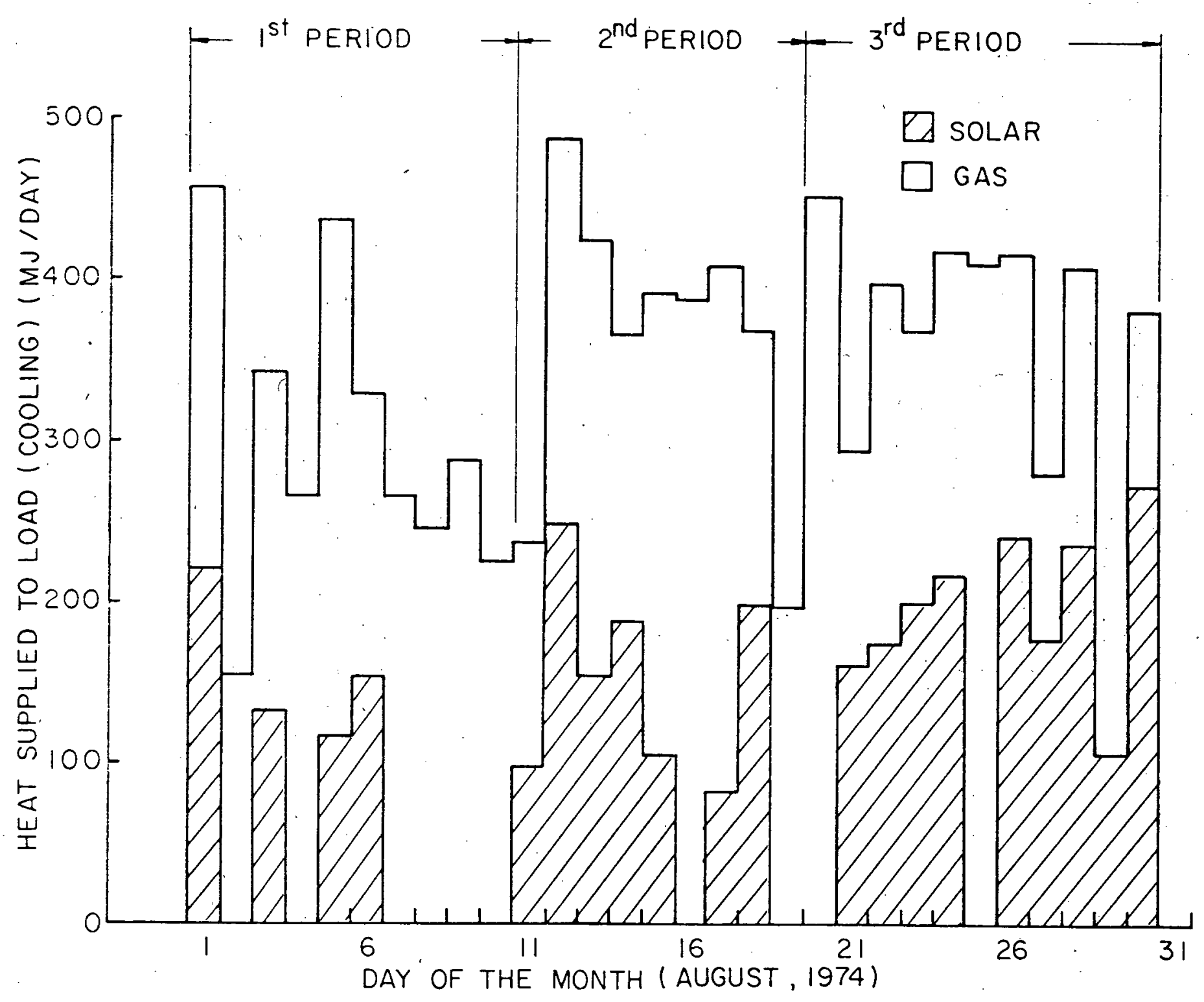

कั

Figure 23. Relative Contributions to Cooling Unit by Solar and Auxiliary 
inlet temperature $\left(82^{\circ} \mathrm{C}, 180^{\circ} \mathrm{F}\right)$. The period of operation from $11-20$ August shows the benefits (Figure 23) of the control instrumentation modification, by increasing the solar energy delivered to the cooling unit from 612.4 MJ to 1048.8 MJ. An equally important improvement was obtained during the last ten days in August by lowering the minimum generator supply temperature from $82^{\circ} \mathrm{C}\left(180^{\circ} \mathrm{F}\right)$ to $77^{\circ} \mathrm{C}\left(170.6^{\circ} \mathrm{F}\right)$. The increase in total solar energy delivered to the cooling unit as a result of the lower generator temperature requirement was $-500 \mathrm{MJ}$ ( $50 \mathrm{MJ} /$ day) . Table 31 summarizes the effects of the control system modifications.

Table 31. Relative Contributions to the Cooling Load by Solar and Auxiliary (August 1974) - Ten Day Totals

\begin{tabular}{|c|cc|c|c|}
\hline Time Span & $\begin{array}{c}\text { * Cooling } \\
\text { Gas }\end{array}$ & $\begin{array}{c}\text { (MJ) } \\
\text { Solar }\end{array}$ & $\begin{array}{c}\text { Per Cent } \\
\text { Solar Cooling }\end{array}$ & $Q_{U}$ (MJ) \\
\hline $1-10$ August & 1011.8 & 364.7 & 26.5 & 2143.5 \\
$10-20$ August & 1442.8 & 745.8 & 34.7 & 2824.1 \\
$21-31$ August & 946.2 & 1192.2 & 55.8 & 3882.6 \\
\hline Total & 3400.8 & 2302.7 & 40.4 & 8850.2 \\
\hline
\end{tabular}

*MJ of heat removed from building by cooling unit (Heat delivered to generator of air-conditioner times coefficient of performance)

Because of the low humidity in CSU SolarHouse I, most of the operating data were obtained during periods when no condensation occurred on the cooling coils. Absence of dehumidification substantially reduced the coefficient of performance of the unit. This is to be expected, because in normal operation, a heat input of $56.5 \mathrm{MJ} / \mathrm{hr}(53,500 \mathrm{Btu} / \mathrm{hr})$ produces sensible cooling of $28.5 \mathrm{MJ} / \mathrm{hr}(26,980 \mathrm{Btu} / \mathrm{hr})$ and $10.6 \mathrm{MJ} / \mathrm{hr}(10,050 \mathrm{Btu} / \mathrm{hr})$ of latent heat removal by condensation. But when the latent load is zero, the COP is thus reduced from 0.69 to 0.50 . This "overfired" situation 
results in the failure of part of the refrigerant to vaporize in the evaporator, thereby flowing as liquid into the absorber.

The average COP of the cooling unit when on auxiliary heat supply was 0.48 (11-30 August). Of particular note, however, is the fact that when solar heat was supplied to the air-conditioner during the same period, an average COP of 0.70 was obtained. This higher value was due to a combination of a lower hot water flow rate to the generator when supplied from solar storage, and a lower average operating temperature of the storage unit compared with auxiliary heat supply temperatures. The heat input rate to the generator thus decreased from $56.5 \mathrm{MJ} / \mathrm{hr}$ (as in the case of gas-supplied heat) to $40 \mathrm{MJ} / \mathrm{hr}$ (average for solar), a heat rate which could normally be expected to deliver $32.3 \mathrm{MJ} / \mathrm{hr}$ of combined sensible and latent cooling (85 per cent of capacity). This heat removal rate nevertheless exceeds the $28.5 \mathrm{MJ} / \mathrm{hr}$ sensible cooling load. The reduced heat supply rate nearly matched the sensible heat removal requirements (condensation being absent), less heat was therefore wasted than when the design heat supply rate was provided, and a favorable solar COP of $0.71(28.5 \mathrm{MJ} / \mathrm{hr}$ divided by $40 \mathrm{MJ} / \mathrm{hr}$ ) was obtained. This value is in good agreement with the daily average solar COP of 0.70 .

Because of the improved COP of solar cooling, the percentage of the cooling requirements (actual heat removed from the building) provided by solar is increased from 36 per cent to over 40 per cent. Figure 24 shows the cooling load met by solar and auxiliary during the month of nugust. Note also Table 31, which shows that under optimum conditions of the last ten days of August, solar supplied almost 56 per cent of the cooling requirements.

The change in the control instrumentation, whereby the inlet generator temperature was lowered (mentioned above), also had the effect of increasing 


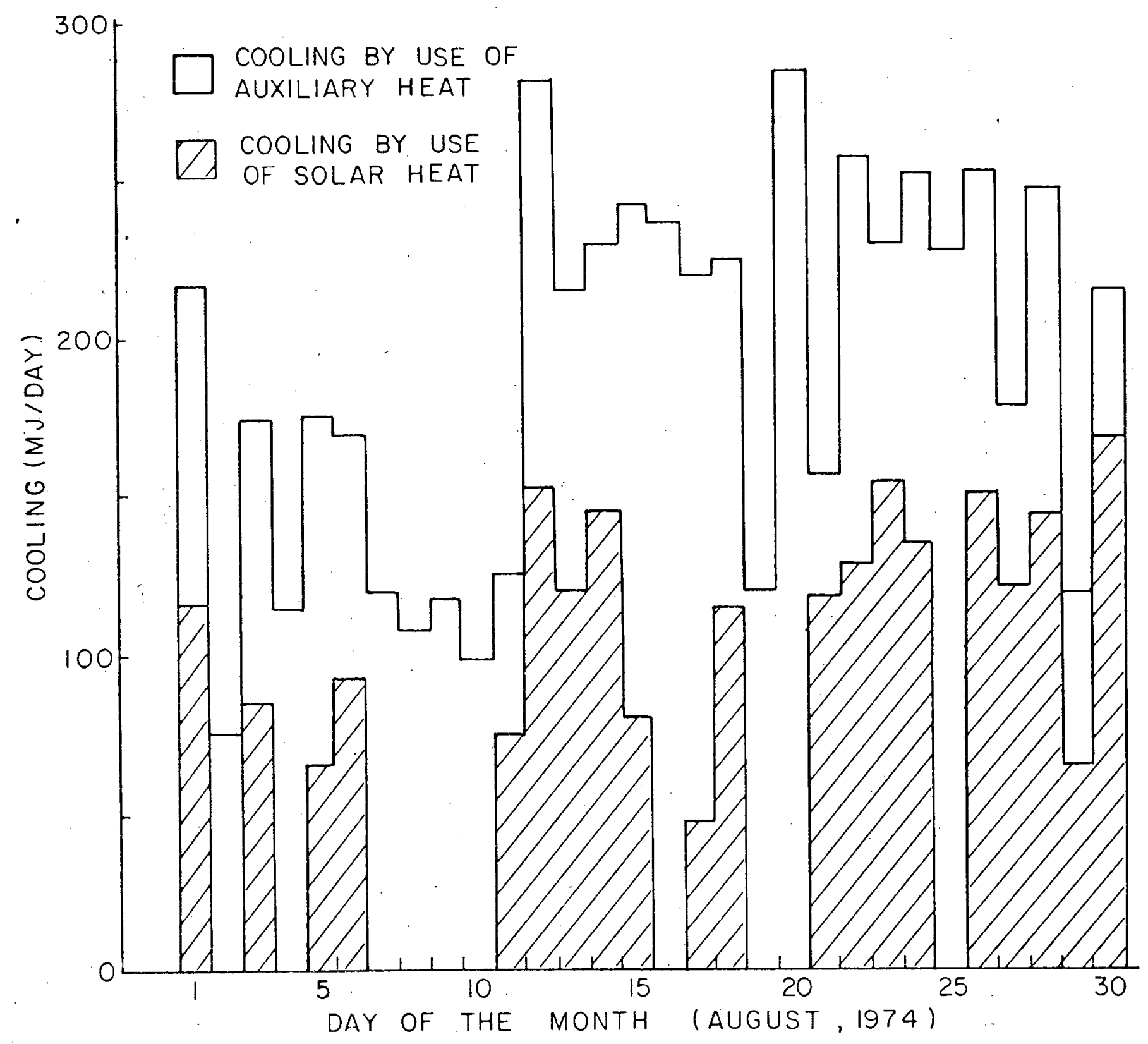

Figure 24. Relative Contributions to Cooling Load by Solar and Auxiliary (August 1974) 
the COP from an average of 0.43 to 0.48 when operated by auxiliary. When solar-operated, -the modification caused an increase in COP from 0.60 to 0.70 .

A much more significant variation in the COP of the cooling unit is due to its operating characteristics, particularly during start-up. Figure 25 is an example of the manner in which cooling performance varies during a period of time. Point $A$ represents the time at which the thermostat initially provides a cooling demand signal (when the absorption unit comes on). The graduál rise of the heat supply curve $\left(Q_{L}\right)$ shows that the initial heat delivered is used partially to warm up the metals and liquids in the unit (prior to operation at normal cooling capacity) for a period as long as fifteen minutes. The delivery of full cooling output is also delayed, as shown by the $Q_{C T}-Q_{L}$ and cop curves.

During days of high cooling demand, the air conditioner encounters these start-up conditions in the morning and then runs nearly continuously at a satisfactory COP. The loss of cooling during start-up is a small fraction of the day's total output, and the COP for the day corresponds to the design value. However, when cooling demand is low, the unit runs intermittently, and the numerous start-ups (as many as 40 in a 24 hour period) require heat to replace the thermal losses occurring during the non-running intervals. Without heat supply, the generator cools off in about ten minutes; so if it runs only ten minutes, the heat losses in one stop-startcycle result in a COP decrease from 0.60 to about 0.30 . It is significant that the start-up heat is added to the building when the cooling unit shuts down, so the cycling of the absorption machine not only causes a lowering of the COP but also adds to the cooling load.

Inefficiencies resulting from intermittent operation of the airconditioner could be greatly reduced by the use of cold-side storage. 


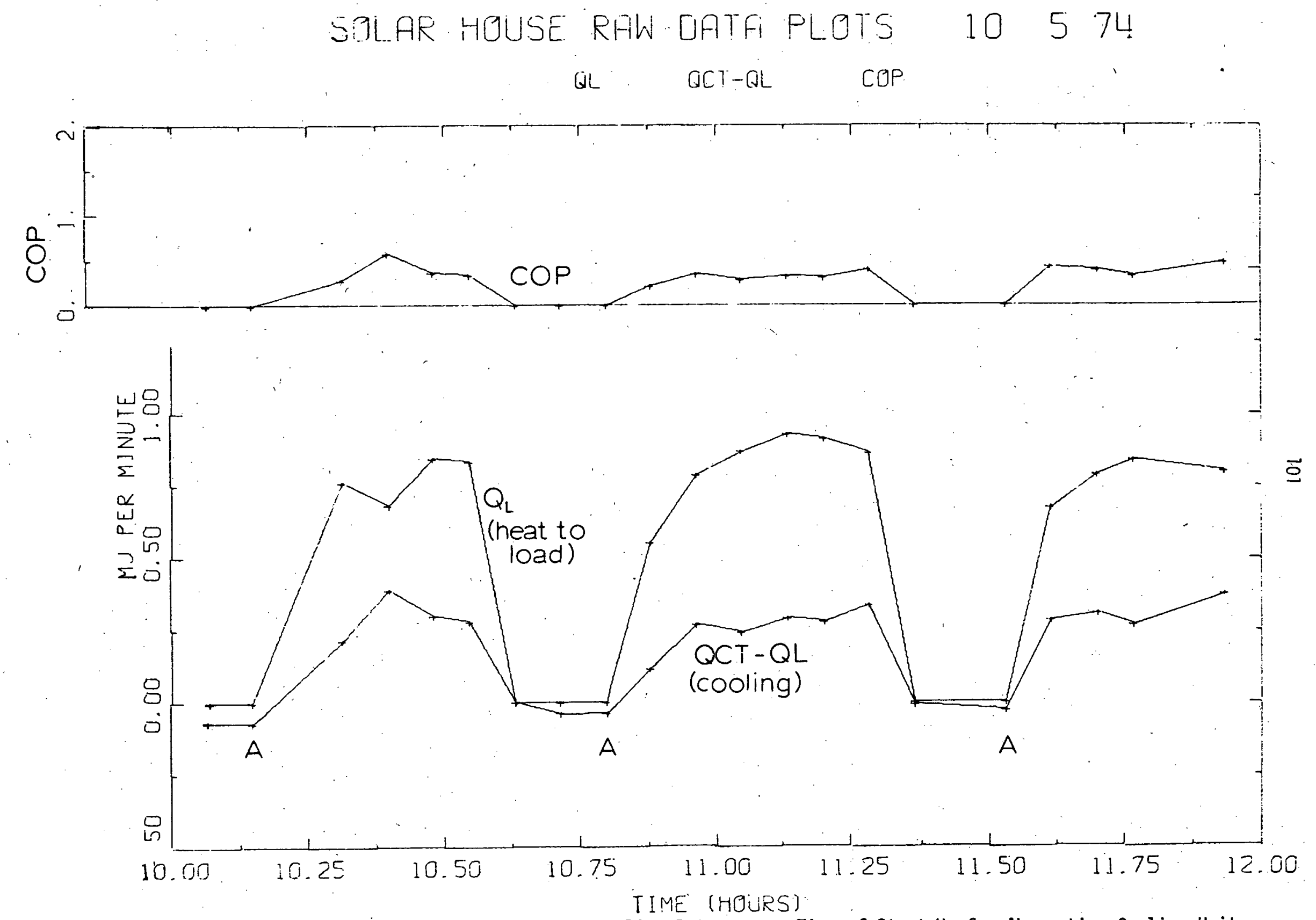

Figure 25. Coefficient of Performance and Cooling Rate versus Time of Start-Up for Absorption Cooling Unit 
The absorption unit could be run continuously during the day so that waste of heat involved in frequent cycling could be avoided.

Additional cooling demands not normally encountered in conventional systems were imposed by the thermal losses from solar components. Measurements 'of losses from the solar thermal storage tank, for example, showed a rate of $0.04 \mathrm{MJ} / \mathrm{hr} \cdot{ }^{\circ} \mathrm{C}$ (approximately double that computed from reported properties of the insulation). During the month of August there was an average heat loss rate of $1.88 \mathrm{MJ} / \mathrm{hr}(1780 \mathrm{Btu} / \mathrm{hr})$. This $45 \mathrm{MJ} /$ day heat loss (1395 MJ/month) can be compared to the hourly sensible heat removed by the cooling unit (ranging from 22.8 to $28.4 \mathrm{MJ}$ ). The air-conditioner must therefore operate for almost two hours per day to remove the heat. added to the house by leakage from storage.

Other heat losses, from the solar preheat tank (hot water system) of $0.492 \mathrm{MJ} / \mathrm{hr}$ (372 MJ/month) and from the surface of the auxiliary hot water tank, pumps, piping, and heat exchangers, added $2619 \mathrm{MJ}$ to the cooling demand in August. Total heat losses from solar equipment aggregate 46 per cent of the total cooling demand of 5703 MJ. Had these losses been el iminated, solar heat would have provided 74.7 per cent of the cooling requirements. Figure 26 indicates the relative amounts of solar and gas heat which. would be delivered to the cooling unit if these thermal losses were el iminated. Losses during the heating season do not appreciably affect auxiliary fuel requirements because the heat is utilized in heating the building directly.

Substantial progress in the reduction of these heat losses has been made in preparation for the next cooling season. These efforts are directed toward better insulation of equipment and the outdoor venting of excess heat from the heating and cooling system components. 


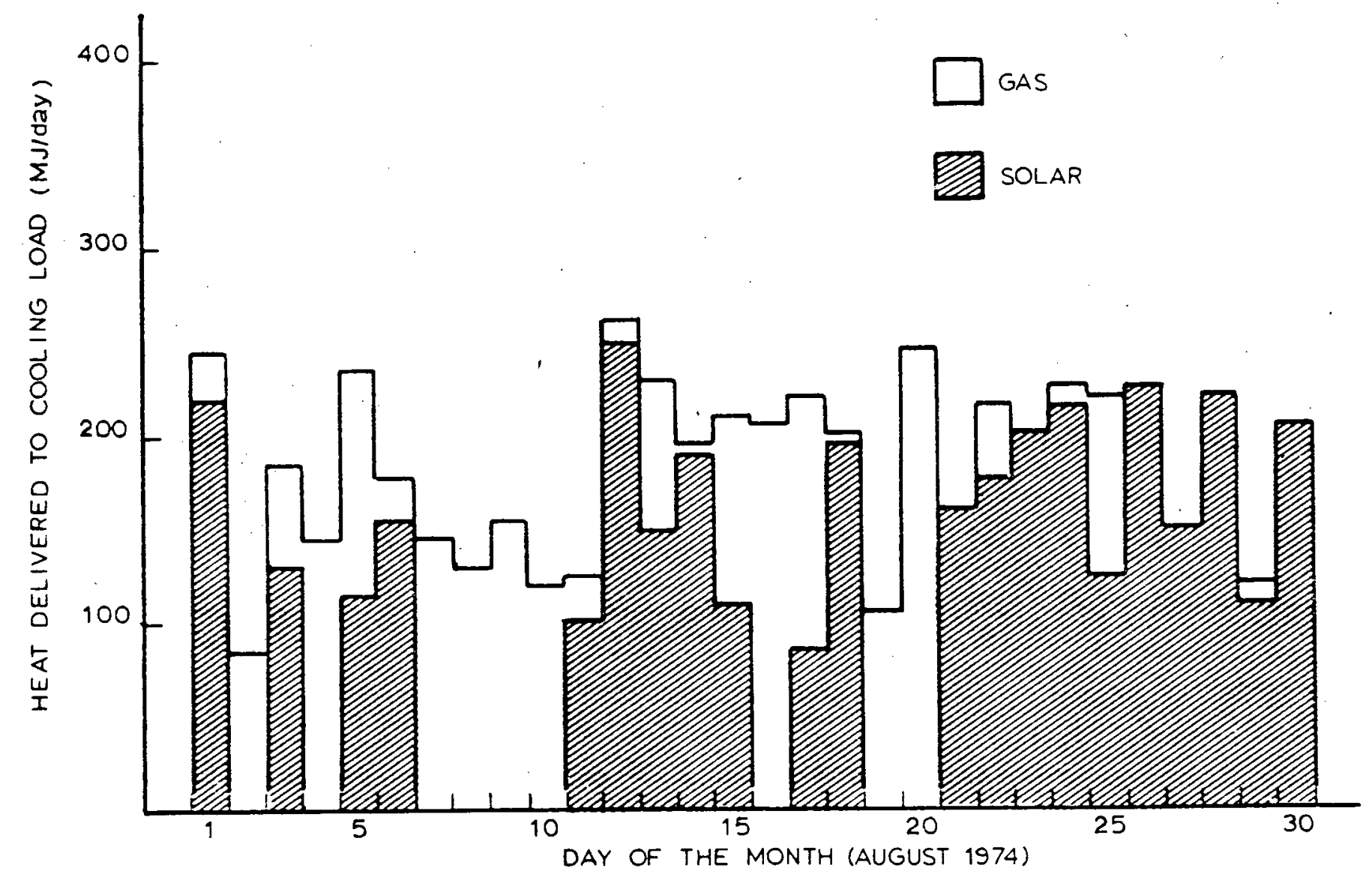

S

Figure 26. Relative Contributions to Cooling Load by Solar and Auxiliary (Normal Cooling Load Oniy) 
Summary of Cooling Performance

Tables 4 through 16 provide values of daily energy totals delivered by solar and auxiliary to the space cooling load. Table 20 lists the per cent solar contributions to the daily energy requirements for space cooling. Numerous factors must be considered in order to evaluate these data. These factors are summarized in Table 32, and will be discussed below. In Table 32, column [1] is the total heat delivered to the cooling machine by auxiliary and solar energy sources. It represents the total heat delivered to the cooling unit. Column [2] is the coefficient of performance (COP) of the absorption cooling unit. The COP is obtained by dividing the actual cooling (heat extracted from the building) by the total heat delivered to the cooling unit. Column [3] represents the total actual cooling of the building air (i.e., the heat extracted from the building); therefore COP equals column [3] divided by column [1].

The results of Table 32 indicate a COP during the cooling season of approximateiy 0.59. As pointed out above, however, there is the potential for a significant decrease in the cooling unit's COP under conditions of low cooling loads. For example, in September 1974, when the cooling load (heat extracted from the building) was only $132.4 \mathrm{MJ} /$ day (down from a seasonal average of $221.6 \mathrm{MJ} / \mathrm{day}$ ), the COP fell to 0.37 . Similarly in October, the cooling load of $85.7 \mathrm{MJ} /$ day yielded a COP of 40.8 .

This lowering of the COP is due to the "warm up" time of the cooling unit (i.e., the time it takes to reach expected cooling performance from the initial input of heat to the generator). See figure 25. When the cooling demand is low, the unit cycles on and off many times during the day, and the unit's COP drops drastically. In the day shown in Figure 25 , the cooling unit cycled on and off 39 times, yielding a COP of 0.4 . 
Table 32. Monthly Averages of Cooling Values

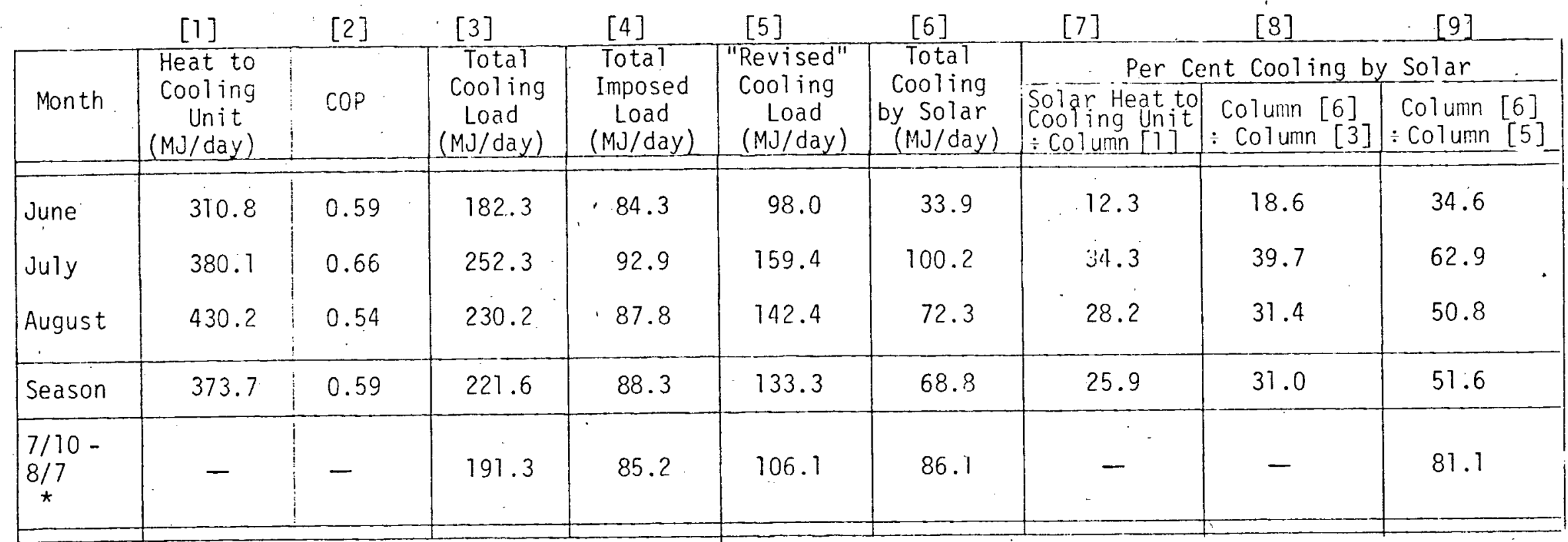

* Special period during which cooling unit was not allowed to run at night 
The per cent cooling by solar (column [7]) is calculated by dividing the total heat delivered by solar to the cooling unit (see Tables 13 through 16) by the total heat (solar and auxiliary) delivered to the cooling unit, column [1]. However, the COP of the absorption cooling unit, when operated by solar was normally higher ( 0.71 ) than the corresponding COP for the auxiliary ( 20.50); again, due to a low latent cooling load at the CSU Solar House I site. With this difference in COP, we can consider a per cent cooling load by solar as the total cooling (heat extracted from the building) by solar, column [6]; divided by the total cooling load, column [3]. This yields an increased per cent load by solar of 25.9 to 31.0 per cent. Another significant factor in the cooling performance is the additional cooling load caused by the heat losses from the solar equipment to the building's interior. This "imposed load" on the cooling load by the solar system heat losses has al ready been described in Table 23 and is listed in column [4] of Table 32 for convenience. If we assume that these heat losses are eliminated (i.e., the solar equipment is located outside of the building's interior such that equipmenit heat losses do not contribute to the cooling load), then we can obtain a "revised" cooling load. This revised cooling load is shown in column [5] of Table 3] and equals column [3] minus column [4].

Under these conditions we can obtain another "per cent cooling by solar" as the total cooling by solar, column [6], divided by the total "revised" cooling load, column [5]. This yiclds a solar contribution to cooling of 51.6 per cent.

A final adjustment to the value of the solar contribution to cooling load can be considered by attempting to limit the absorption unit cycling and consequent reduction in COP, discussed above. During the period io July through 7 August, the cooling unit was deenergized during night 
time periods. This eliminated much of the cycling of the unit (the Solar House frequently had very low cooling loads at night due to the low humidity conditions in Fort Collins).

Table 32 shows the effect of this experiment on the bottom row of the table. Using the cooling load based on no solar equipment heat losses contribution to the cooling load (i.e., the "revised" cooling load in column [5] and the total cooling by solar in column [6]), we obtain a solar contribution to the cooling load of 81.1 per cent. 